\title{
C-Jun N-Terminal Kinase Signaling Guides the Migration of Interneurons During Cortical Development
}

\author{
Abigail K. Myers
}

Follow this and additional works at: https://researchrepository.wvu.edu/etd

\section{Recommended Citation}

Myers, Abigail K., "C-Jun N-Terminal Kinase Signaling Guides the Migration of Interneurons During Cortical Development" (2017). Graduate Theses, Dissertations, and Problem Reports. 6285.

https://researchrepository.wvu.edu/etd/6285

This Dissertation is protected by copyright and/or related rights. It has been brought to you by the The Research Repository @ WVU with permission from the rights-holder(s). You are free to use this Dissertation in any way that is permitted by the copyright and related rights legislation that applies to your use. For other uses you must obtain permission from the rights-holder(s) directly, unless additional rights are indicated by a Creative Commons license in the record and/ or on the work itself. This Dissertation has been accepted for inclusion in WVU Graduate Theses, Dissertations, and Problem Reports collection by an authorized administrator of The Research Repository @ WVU.

For more information, please contact researchrepository@mail.wvu.edu. 


\title{
c-Jun $\mathrm{N}$-terminal kinase signaling guides the migration of interneurons during cortical development
}

\author{
Abigail K Myers \\ Dissertation submitted to the School of Medicine \\ at West Virginia University \\ In partial fulfillment of the requirements for the degree of \\ Doctor of Philosophy in \\ Neuroscience \\ Eric S Tucker, Ph.D., Chair \\ Peter Mathers, Ph.D. \\ Ariel Agmon, Ph.D. \\ Scott Weed, Ph.D. \\ Shuo Wei, Ph.D.
}

Neuroscience Graduate Program

Morgantown, WV

2017

Keywords: c-Jun N-Terminal Kinase (JNK), cortical interneurons, developmental cortical disorders, migration

Copyright 2017 Abigail K Myers 


\title{
Abstract \\ c-Jun $\mathrm{N}$-terminal kinase signaling guides the migration of interneurons during cortical development
}

\author{
Abigail K Myers
}

The cerebral cortex, responsible for higher order functions, is a layered structure that relies on the precise wiring of two main types of neurons, excitatory neurons and inhibitory interneurons. In order to maintain a critical balance of excitation and inhibition in the cortex, a smaller, yet very diverse, population of cortical interneurons provides inhibition to a much larger cohort of excitatory neurons. This makes interneurons a vulnerable population where the slightest perturbations to their location and wiring with excitatory neurons can contribute to the acquisition of neurological disorders like autism, schizophrenia and epilepsy.

During development, cortical interneurons migrate tangentially from the medial and caudal ganglionic eminences in the ventral forebrain to the cortex. Unlike cortical excitatory neurons that are born in the ventricular zone of the dorsal forebrain and migrate radially on radial glial scaffolding, cortical interneurons are born in the ventral forebrain and migrate tangentially, primarily using molecular guidance cues in the extracellular environment to enter, navigate, and form streams in the nascent cerebral cortex. As development proceeds, interneurons depart from migratory streams on diagonal or radial trajectories in order to populate the cortical plate, where they eventually segregate into layers and make synaptic connections with other cortical neurons. Signaling pathways allow interneurons to express the correct complement of cell surface guidance receptors, transduce signals associated with the activation of those receptors, and facilitate the rearrangement of their cytoskeleton in order to aid in their travel. These processes are essential for the proper entrance of cortical interneurons into the cerebral cortex, their exit from migratory streams, and ultimately their layering in the cortical plate. Yet, how intracellular signaling pathways govern these processes remains largely unknown.

The c-Jun N-terminal kinase (JNK) signaling pathway, primarily known for its role in cell death, plays a large role in cell migration by activating downstream targets that modify the cytoskeleton and cell architecture. However, its importance in cortical interneuron migration has not been established. This work, organized into four main chapters including two main data chapters and an appendix, provides the first evidence that cortical interneurons use JNK signaling to enter and navigate in the developing cerebral cortex. In the first data chapter, we establish that Jnk is present within cortical interneurons and Jnk 1 is required for their entry into the cerebral cortex between embryonic day (E) E12.5 and E13.5 of mouse development. The second data chapter provides evidence that JNK signaling is important for maintaining migratory streams and that pharmacological inhibition or genetic ablation of Jnk from interneurons incites their exit from migratory streams and subsequent entry into the cortical plate between E14.5 and E15.5. Further, the work provided in the Appendix indicates disruption to the localization of Jnkphosphorylated doublecortin, a microtubule associating protein important for branching of the leading processes of the migrating interneurons. Together, this work suggests that JNK signaling plays a critical role during cortical interneuron migration and may provide insight into how neurodevelopmental disorders like epilepsy, autism and schizophrenia could arise. 


\section{Dedication}

To my family, friends, and mentors, thank you for all your love and support throughout all of my education 


\section{Acknowledgements}

I would like to take a few moments to thank the many people that have helped me throughout my career as a PhD graduate student.

To my advisor, Dr. Eric Tucker, thank you for teaching me everything you know about lab techniques, experiments, and publishing papers, as well as giving me the opportunity to attend conferences and introducing me to colleagues to help me network with others. Thank you for working with me as a team to train other students that have come into and through the lab and for teaching me how to manage and run a lab. Last, but not least thank you for taking me on as your first graduate student. I know this experience hasn't been without its ups and downs, but thank you for sticking with me and working through all the challenging points. You have taught me so much about the field, and I have grown so much from this experience.

To my committee members, Dr. Pete Mathers, Dr. Scott Weed, Dr. Aric Agmon, and Dr. Shuo Wei, thank you for all your guidance, support, and feedback on projects and papers.

To Dr. Dey, thank you for all your support and for providing a listening ear, direction, and feedback when I had a problem or needed advice. I genuinely appreciated our meetings and talks and everything that came out of them.

To Dr. Randy Kulesza and my teaching professors, thank you for giving me the opportunity to teach, for being patient with me while I learned the best strategies to teach, and for teaching me the most up-to-date ways of keeping students engaged.

To my past and current labmates (John, Danielle, Neha, Bree, Emily, Kathryn, Katie, Emily, John, Kelly, Michael, Steven, Jessica, Skye, Catherine), thank you for all your help with the mouse colony and experiments. I am so glad every one of you decided to join the lab. Each one of you has taught me something new, and I am grateful that I have had the opportunity to work with all of you. Thank you for being patient with me during hectic experiment times and defense times, and most of all thank you for your support and friendship. I wish you all the best of luck with your projects and future endeavors.

To Dr. Amanda Ammer and Dr. Karen Martin, thank you for your help with everything confocal. Thank you for including me in training sessions, being my never-ending source of answers when things went wrong with the confocal imaging, and for making my work environment flexible with Imaris. I loved working with you both.

Thank you to my graduate student colleagues and friends (both inside and outside the neuroscience program). I would not have made it this far without the many lunches, dinners, drinks, walks, talks, and hugs. I never thought that I would have grown so much while I was here at WVU, but I had the best friends to help me through everything I faced. I am so grateful for every single one of our friendships.

Thank you to my family and family friends for your guidance, support, and love throughout all of my schooling. Specifically, I would like to thank my parents, Sue and Tom, my brother, Aaron, my sister-in-law, Natalie, my Aunt Donna, Grammie, my best friend, Liz, and my love, Russ, for always being there to celebrate my successes and to encourage me through my struggles. Words cannot describe how much your unwavering love and kindness means to me. 


\section{Table of Contents}

Abstract

Dedication

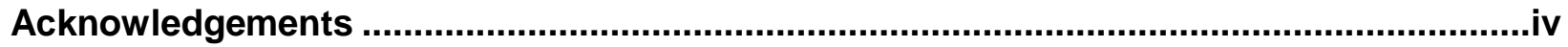

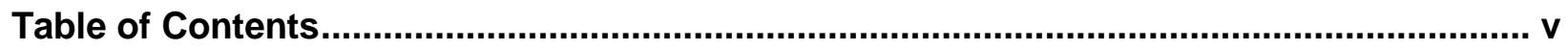

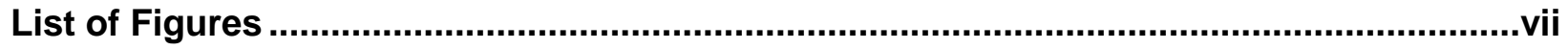

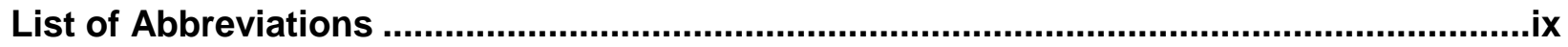

Chapter 1: Introduction and Literature Review ........................................................ 1

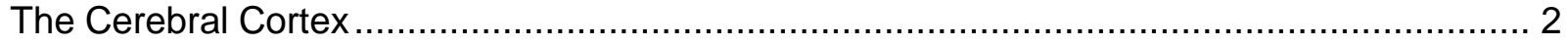

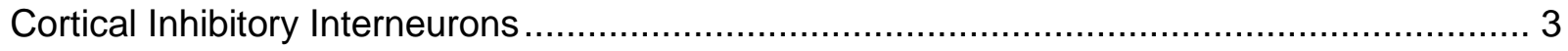

Parvalbumin-expressing interneurons .................................................................... 4

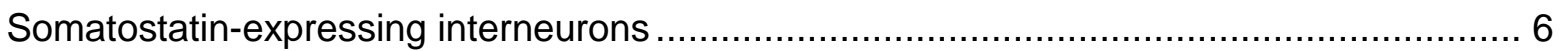

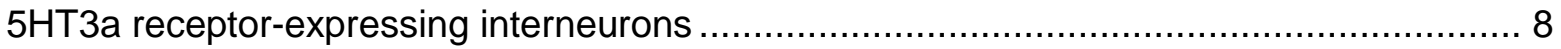

The Ventral Forebrain and Development of Cortical Interneuron Populations ......................... 9

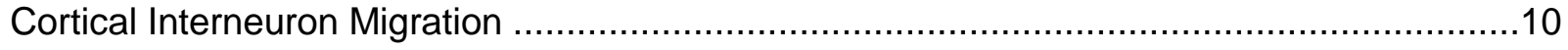

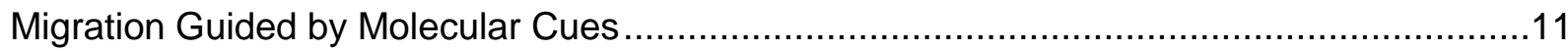

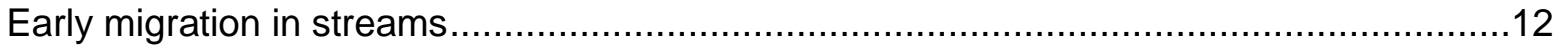

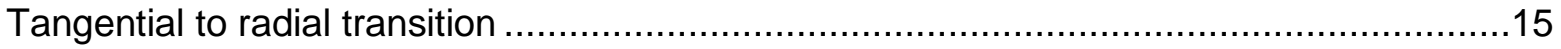

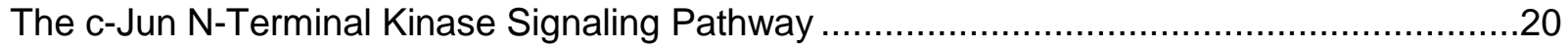

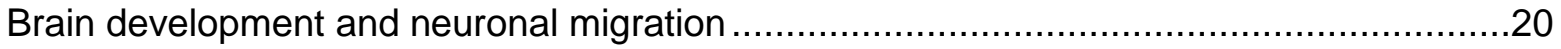

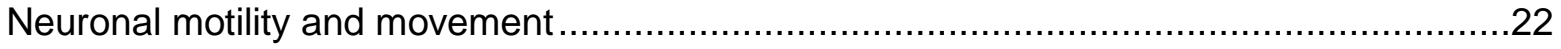

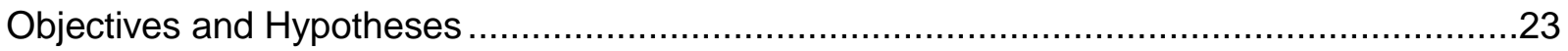

\section{Chapter 2: Cortical Interneurons Require Jnk1 to Enter and Navigate the Developing}

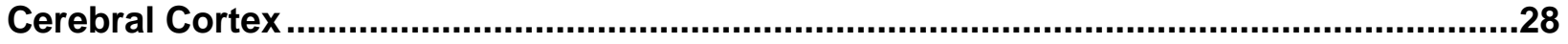

Abstract

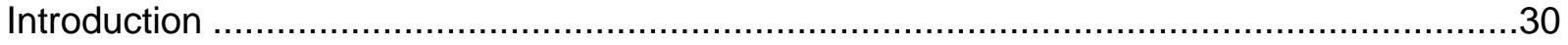

Materials and Methods

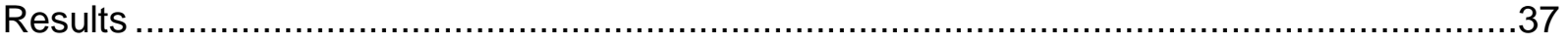

Interneurons express Jnk proteins as they first enter the cortical rudiment.........................37

Interneuron entry into the cortical rudiment requires intact JNK signaling.........................38

JNK inhibition impairs directed migration of cortical interneurons into the cortical rudiment 40

Jnk1 regulates cortical interneuron migration at the cortical entry zone in vivo...................43

Cortical interneurons have a cell-intrinsic requirement for Jnk1 in vivo.............................46

Integrity of cortical migratory streams depends on JNK signaling in vivo...........................49 


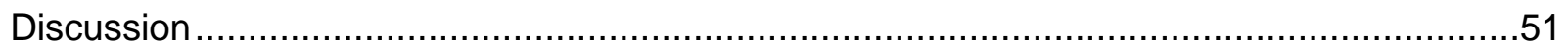

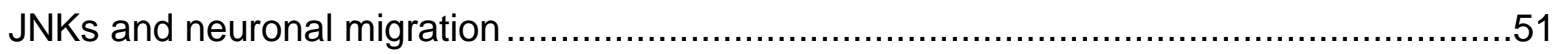

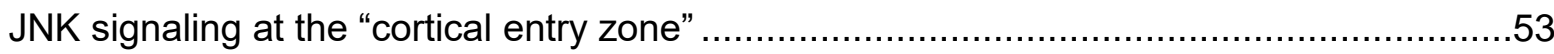

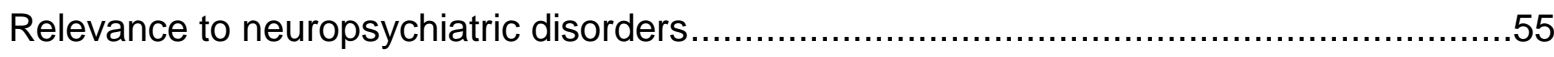

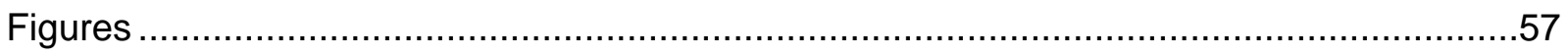

Chapter 3: JNK Signaling Preserves Cortical Interneuron Stream Integrity and Radial

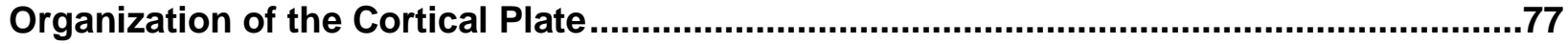

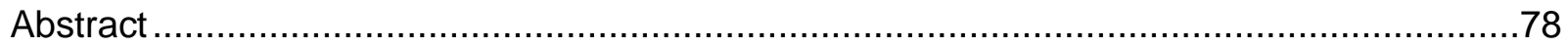

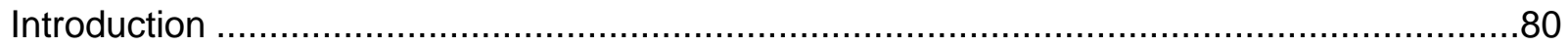

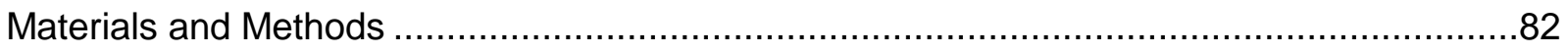

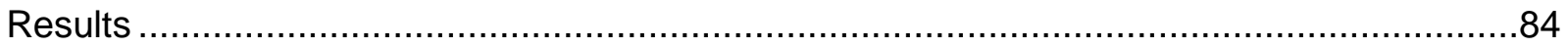

JNK signaling maintains the integrity of cortical interneuron migratory streams ...............84

Stream integrity can be partially restored after disruption of JNK signaling .....................87

Pharmacological loss of JNK signaling causes cortical interneurons to leave the subventricular zone stream and spend more time in the cortical plate.

Genetic loss of JNK signaling causes cortical interneuron stream dispersion and cortical plate accumulation

Genetic loss of JNK signaling causes interneuron clustering in the cortical plate and intermediate zone regions.

Cortical interneuron clusters form from the marginal zone stream in JNK deficient cortices

Cortical interneuron clusters originate in regions of radial glial disruption.......................94

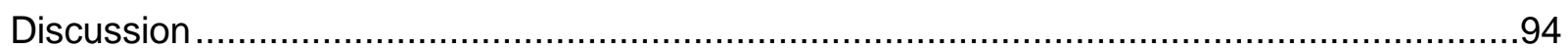

JNK signaling and cortical interneuron migration................................................ 95

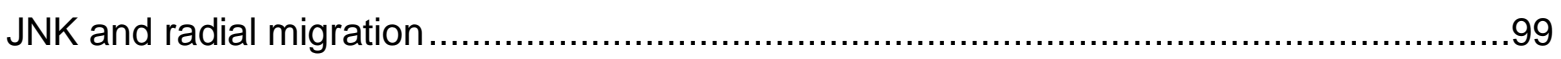

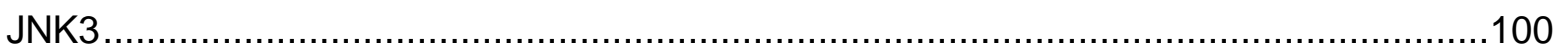

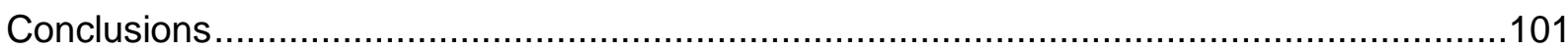

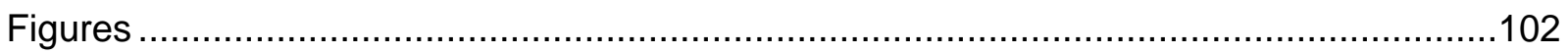

Chapter 4: Summary and Future Directions.................................................................114

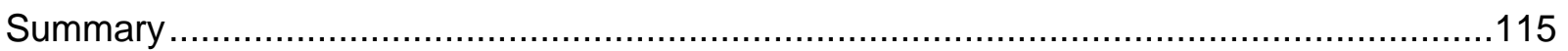

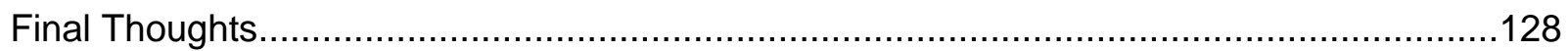

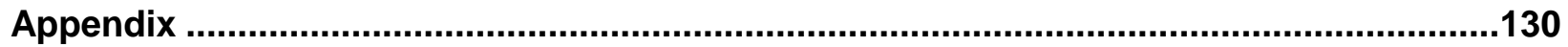

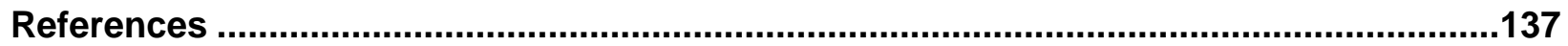

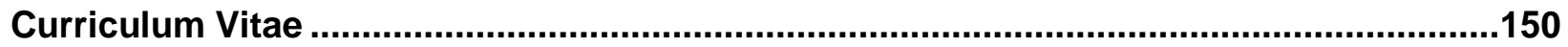




\section{List of Figures}

\section{Chapter 1: Introduction and Literature Review}

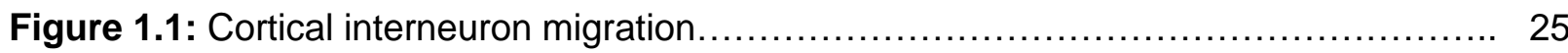

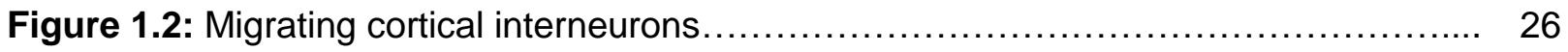

Figure 1.3: The c-Jun-N-terminal kinase $(J N K)$ pathway............................ 27

Chapter 2: Cortical Interneurons Require Jnk1 to Enter and Navigate the Developing Cerebral Cortex

Figure 2.1: Early arriving cortical interneurons express Jnk proteins.

Figure 2.2: Cortical interneurons require JNK activity to enter the developing cerebral cortex.

Figure 2.3: Disruption of cortical interneuron migration by pharmacological inhibition of JNK signaling is specific and reversible....

Figure 2.4: Migratory properties of cortical interneurons are perturbed following SP600125 treatment at E12.5.

Figure 2.5: Genetic ablation of Jnk1 impairs migration of cortical interneurons in vivo.......

Figure 2.6: Unlike Jnk1, genetic ablation of Jnk2 does not disrupt migration of cortical interneurons in vivo.

Figure 2.7: Cortical interneurons have a cell-intrinsic requirement for JNK signaling to migrate through the cortical entry zone in vivo.....

Figure 2.8: In vitro migration of cortical interneurons from explants of MGE tissue does not rely on JNK signaling....

Figure 2.9: Cortical interneurons lacking Jnk1 and Jnk2 disperse from migratory streams and adopt branched morphologies in vivo.

Figure 2.10: Migration of cortical interneurons through the cortical entry zone in vivo requires intact JNK signaling, largely mediated by interneuron expressed Jnk1.....

Movie 2.1: Live imaging of cortical interneuron migration through the cortical entry zone at E12.5 in a control slice.

Movie 2.2: Live imaging of cortical interneuron migration through the cortical entry zone at E12.5 in an SP600125-treated slice. 


\section{Chapter 3: JNK Signaling Preserves Cortical Interneuron Stream Integrity and Radial Organization of the Cortical Plate}

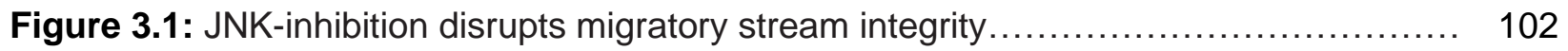

Figure 3.2: Removal of the JNK inhibitor improves migratory streams integrity ............. 104

Figure 3.3: Cortical interneuron streams show trends of recovery in the ventricular zone

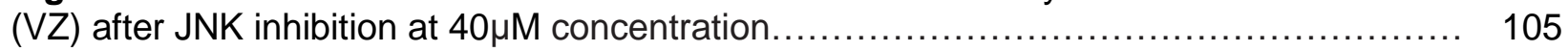

Figure 3.4: Tangential progression in migratory streams requires JNK activity .............. 106

Figure 3.5: Cortical interneurons migrating within the SVZ of pharmacologically treated slices have decreased displacement and less straight tracks when compared to controls..

Figure 3.6: Genetic loss of JNK signaling from cortical interneurons disrupts migratory stream integrity

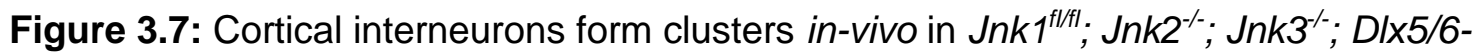
CIE cortices at E15.5.

Figure 3.8: Cortical interneurons form clusters originating from the marginal zone stream..

Figure 3.9: Cortical interneurons in cTKO cortices accumulated faster and more abundantly than cortical interneurons in CTH and CDKO cortices over 12 hours.....

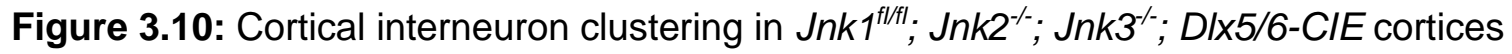
coincides with disruption to radial glia.

\section{Appendix:}

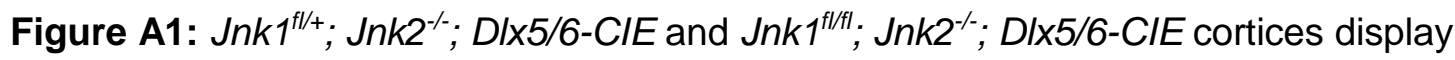
similar advancement and radial distribution at E15.5.

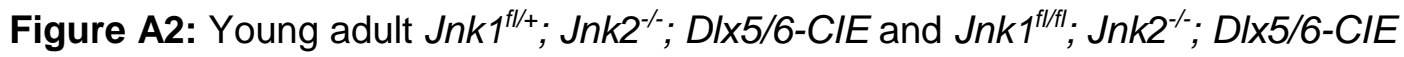
cortices share similar distributions of cortical interneurons.

Figure A3: Similar to Jnk2, genetic ablation of Jnk3 at E13.5 does not disrupt cortical interneuron migration

Figure A4: Jnk-phosphorylated Dcx labeling is shifted to the cell body in cortical

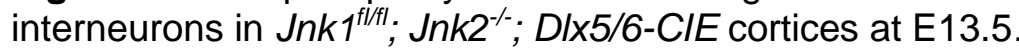

Figure A5: Calbindin labeling is reduced in cortical interneurons and absent in axon

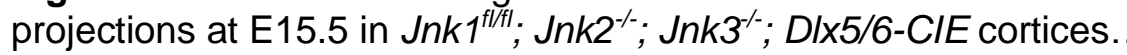




\section{List of Abbreviations}

5HT3aR- 5-Hydroxytryptamine-3a Receptor

ADP- Adenosine Diphosphate

AMPA- a-Amino-3-Hydroxy-5-Methyl-4-Isoxazolepropionic Acid Receptor

Ant1- Adenine Nucleotide Transferase 1

ApoER2- Apolipoprotein-E Receptor 2

Arl13b- ADP-Ribosylation Factor-Like Protein 13B

ATP-Adenosine Triphosphate

CCK- Cholecystokinin

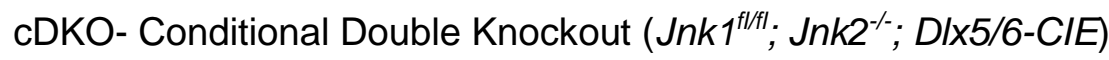

CGE- Caudal Ganglionic Eminence

CP- Cortical Plate

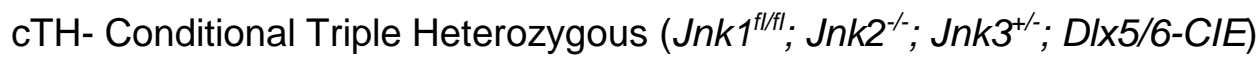

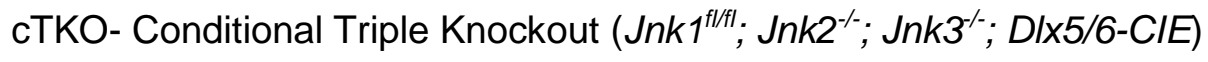

CXCL12- CXC- Ligand 12

CXCR4/7- CXC-Receptor Motif 4/7

Dab1- Disabled 1

DCX- Doublecortin

DIx5/6-CIE- DIx5/6-Cre-Ires-EGFP

DMSO- Dimethyl Sulfoxide

E- Embryonic Day

ErbB4- Erb-B2 Receptor Tyrosine Kinase 4

GABA- Gamma-Aminobutyric Acid

GAD67- Glutamic Acid Decarboxylase 67

GFP- Green Fluorescent Protein

GIN- Green Fluorescent Protein-Expressing Inhibitory Neurons

IFT- Intraflagellar Transport

IZ- Intermediate Zone

JIP- JNK-Interacting Protein 
JNK- c-Jun N-Terminal Kinase

LgDel- Large Deletion

LGE- Lateral Ganglionic Eminence

Map2k4/7- Mitogen Protein Kinase Kinase 4/7

Map3k12- Mitogen Protein Kinase Kinase Kinase 12

MAPK- Mitogen Activated Protein Kinase

MARKS- Microtubule Affinity Regulating Kinases

MeCP2- Methyl CpG Binding Protein 2

MGE- Medial Ganglionic Eminence

MKK4/7- Mitogen-Activated Protein Kinase Kinase 4/7

MZ- Marginal Zone

NMDA- N-Methyl-D-Aspartate Receptor

NPY- Neuropeptide-Y

P- Postnatal Day

PBS- Phosphate Buffered Saline

PKA- Protein Kinase A

PTEN- Phosphatase and Tensin Homolog Gene

REM- Rapid Eye Movement

SCG10- Superior Cervical Ganglion 10

SD- Standard Deviation

SDF-1- Stromal Derived Factor-1

SHH- Sonic Hedgehog

SVZ- Subventricular Zone

TAOK2- TAO Kinase 2

VIP- Vaso-Intestinal Peptide

VZ- Ventricular Zone 
Chapter 1: Introduction and Literature Review 


\section{The Cerebral Cortex}

The cerebral cortex, specifically the neocortex, controls our ability to sense and respond to our environment through decision-making, movement, communication, vision, hearing, and sensory perception. It is comprised of, in a broad sense, two mitten shaped hemispheres, each comprising five lobes (frontal, parietal, occipital, temporal, and limbic), organized layers of gray matter, and white matter tracts. Alfred Campbell was one of the first to relate structure to function, identifying neocortical regions important for motor function, vision, audition, and sensation (Campbell, 1905; Loukas et al., 2011). This work was followed by Korbinian Brodmann who performed a detailed histological characterization of the cortex, identifying and describing the cellular composition of numerous areas (Loukas et al., 2011). These well-defined cortical regions are known today as Brodmann's areas (Loukas et al., 2011).

The neocortex is organized into six-layer structure composed of two types of neurons. Excitatory neurons use glutamate as a neurotransmitter and relay neural information over long distances both within and outside of the cerebral cortex and are therefore also known as projection neurons. These neurons are comprised of primarily pyramidal neurons that arise from a diverse group of progenitors in the dorsal (cortical) ventricular zone and subventricular zone (Stancik et al., 2010). They migrate radially to their proper laminar position during cortical development, populating the cortex in an inside-out manner (the earliest born excitatory neurons reside in the deepest layers of the cortex while later born neurons reside in more superficial layers; Rakic, 1972, 1974, 1978). In contrast, inhibitory neurons, also known as cortical interneurons, produce and release the neurotransmitter gamma-aminobutyric acid (GABA) and critically gate the activity of excitatory neurons despite being outnumbered about four to one (Rudy et al., 2011). During development, cortical interneurons are born in the ventral forebrain and migrate tangentially to populate the cortex (Anderson et al., 2001; Wichterle et al., 2001; Xu et al., 2004; Miyoshi et al., 2010). Proper positioning and circuit formation of these two neuronal 
types is imperative for normal behavior and cognition (Rakic, 1975; Benes et al., 1991). Perturbations in the development of excitatory neurons and interneurons underlie a large number of human neurodevelopmental disorders. Disrupted positioning of inhibitory interneurons in particular during development may alter their connectivity within the mature cortical circuit and interfere with the normal excitatory-inhibitory balance within the cerebral cortex (Isaacson and Scanziani, 2011). Alterations in excitatory-inhibitory balance within the cerebral cortex are thought to underlie severe neurodevelopmental disorders, including schizophrenia, epilepsy, and autism (Cho et al., 2006; Bozzi et al., 2012; Snijders et al., 2013). Recently, these disorders were termed interneuronopathies to acknowledge the pathological development of cortical interneurons in conjunction with the abnormal development of excitatory neurons (Kato and Dobyns, 2005).

\section{Cortical Inhibitory Interneurons}

Cortical interneurons are not a single neuronal type, but an extremely diverse and important population of neurons in the cerebral cortex (Rudy et al., 2011). Together, they prevent hyperexcitability by inhibiting excitatory neurons, and refine and shape the electrical activity of cortical circuits to promote diverse functions, including working memory and attention (Canuet et al., 2011; Murray et al., 2015; Kim et al., 2016b). They account for approximately $20 \%$ of all cortical neurons, making them a vulnerable cell population (Rudy et al., 2011). Subtypes of cortical interneurons differ in molecular composition (parvalbumin, somatostatin, 5HT3aRexpressing interneurons constitute three diverse groups), electrophysiological properties (fastlate-, quasi-fast-, non-fast- and irregular-spiking, and intrinsically bursting), synaptic contact area with excitatory neurons (cell body, dendrites, or initial axon segment), and layer location (Flames and Marín, 2005; Rudy et al., 2011; Chu and Anderson, 2015). In recognition of their remarkable heterogeneity, many efforts have been made to classify cortical interneurons based on their morphology, gene expression profile, neurotransmitter or protein expression profile, and 
function (Kawaguchi, 1995; Kawaguchi and Kubota, 1997; Ascoli et al., 2008; Rudy et al., 2011; Chu and Anderson, 2015).

The wide diversity of interneurons suggests a unique role for each individual type (Kawaguchi, 1995; Kawaguchi and Kubota, 1997; Ascoli et al., 2008; Rudy et al., 2011). Additionally, studies investigating the underlying pathology in severe neurological disorders have found striking decreases and irregularities in certain populations of cortical interneurons, which further suggests that different subsets could play distinct roles that do not overlap and may account for some of the heterogeneity seen in cortical disorders (Lewis et al., 2012; Snijders et al., 2013; Jacob, 2016). The precise function of individual subsets of cortical interneurons in the normal brain or the ability for each of these populations to compensate or replace one another in disease states is not well understood (Kawaguchi and Kubota, 1993, 1996, 1997; Ascoli et al., 2008).

\section{Parvalbumin-expressing interneurons}

Parvalbumin-expressing interneurons are the most abundant subtype in the cortex and have been highly implicated in neurological disease (Schwaller et al., 2004; Gill et al., 2010; Curley et al., 2011; Akakin et al., 2012; Dutton et al., 2012; Lewis et al., 2012; Meechan et al., 2012; Kim et al., 2016a). Parvalbumin interneurons are fast-spiking neurons that reside in all cortical layers except layer 1 (Kawaguchi and Kubota, 1993; Taniguchi et al., 2013). There are actually two major types of parvalbumin-expressing interneurons, each with a distinct morphological and electrophysiological characteristics (Kawaguchi and Kubota, 1997). The first are basket cells, they have a large plexus of axons that extend and target the cell body and proximal dendrites of cortical pyramidal cells (Szentagothai, 1975). In contrast, chandelier cells have distinct morphological features with cartridges of synaptic boutons at the end of each axonal branch that target the initial axon segment of excitatory neurons (Szentagothai, 1975). The 
electrophysiological properties of chandelier cells are distinct from basket cells despite having fast-spiking properties like basket cells (Szabadics et al., 2006; Glickfeld et al., 2009; Woodruff et al., 2009). It remains unclear whether chandelier cells depolarize or hyperpolarize excitatory neurons (Glickfeld et al., 2009; Woodruff et al., 2009, 2011). Early studies on chandelier cells have predominately found them to have a depolarizing effect on excitatory neurons, atypical of cortical interneurons (Szabadics et al., 2006; Woodruff et al., 2009). Contrary to these data, another study found that chandelier cells may have a hyperpolarizing, and therefore inhibitory effect, on excitatory neurons (Glickfeld et al., 2009). The latter study indicated that methods used in the previous study to record these neurons were not sufficient to maintain membrane potential or intracellular ion concentrations of excitatory neurons (Glickfeld et al., 2009). Further, studies by Woodruff and colleagues (2011) have further complicated our understanding of these cells, they found chandelier cells may play a dual role at the initial axon segment synapse. Their data suggest chandelier cells may either hyperpolarize or depolarize a postsynaptic excitatory neuron based on the membrane potential of the excitatory neuron (Woodruff et al., 2011). Together, these studies indicate that parvalbumin-expressing interneurons provide a wide variety of inputs to excitatory neurons that thereby intricately shape neural activity in the cortex.

Dysfunction in parvalbumin-expressing interneurons has been implicated in many neurodevelopmental disorders including autism, schizophrenia, and epilepsy (Schwaller et al., 2004; Dutton et al., 2012; Lewis et al., 2012; Sgadò et al., 2013). Studies report many different parvalbumin-expressing neuron pathologies that can underlie these disease states including a decrease in the number, improper laminar position, and incomplete maturity of parvalbuminexpressing neurons in the prefrontal cortex, a location where reasoning, emotion, and decision making is believed to occur (Gill et al., 2010; Curley et al., 2011; Meechan et al., 2012; Hashemi et al., 2016). Specifically, Curley and colleagues (2011) found decreased expression of GAD67 mRNA, an enzyme that converts glutamic acid to GABA, without alterations in the number of 
parvalbumin interneurons in patients with schizophrenia. The altered expression of GAD67 was correlated with irregularities in gamma oscillations, brain waves that correlate with focus and attention and often appear during REM sleep (Cho et al., 2006; Curley et al., 2011). Other investigators have reported a loss of GABAergic neurons in epilepsy, citing its loss as a probable cause of hyperexcitability (Schwaller et al., 2004; Gill et al., 2010).

\section{Somatostatin-expressing interneurons}

Somatostatin expressing interneurons, located in layers II through VI, have projections to the superficial layers of the cerebral cortex that provide inhibition to the distal apical dendrites of excitatory neurons (Oliva Jr et al., 2000; Ma et al., 2006; Fino and Yuste, 2011). This subtype of interneuron, which is responsible for providing local feedback inhibition to excitatory neurons, demonstrates diverse electrophysiological properties and morphologies (Fino and Yuste, 2011). Somatostatin-expressing cortical interneurons have been characterized using several different mouse lines including GIN, X94, and X98 lines that together account for about $50 \%$ of the total population of somatostatin neurons (Ma et al., 2006). The GFP-expressing inhibitory neurons (GIN) mouse line was the first line developed with GFP expression in somatostatin-expressing interneurons residing in the hippocampus and cortex (Oliva Jr et al., 2000). In the somatosensory cortex, the GIN line labeled interneurons residing in layers II, IV, and upper layer V (Oliva Jr et al., 2000). Bipolar neurons were the most prevalent labeled neuron in this line followed by cortical interneurons with a pyramidal shaped soma located primarily in layer II (Oliva Jr et al., 2000).

Further characterization of somatostatin interneurons was done through the X94 and X98 lines created by Ma and colleagues (Ma et al., 2006). The X94 and X98 lines were found to have a similar distribution of somatostatin-expressing neurons to the GIN line, but additionally labeled somatostatin cortical interneuron populations that were sparse or absent in the GIN line (Oliva 
Jr et al., 2000; Ma et al., 2006). The X94 mouse line labeled many somatostatin interneurons in layer IV and lower layer V (Ma et al., 2006). The X98 line labeled similarly to the X94, but labeled more somatostatin interneurons residing in layer VI with no labeling in layer IV (Ma et al., 2006).

Somatostatin interneuron morphology reflected their location, targets, and electrophysiological properties. Somatostatin cortical interneurons found in layer IV were a novel finding by Ma and colleagues (2006). These cortical interneurons displayed short range axons and dendrites, and exhibited stuttering and quasi-fast-spiking electrophysiological properties which Ma and colleagues (2006) state may have led to previous mischaracterization of this subtype. A more recent study confirmed these results and found that somatostatin interneurons in layer IV are responsible for inhibiting fast-spiking interneurons and the disinhibition of local thalamocortical circuitry (Xu et al., 2013). Additionally, somatostatin interneurons residing in layer V and VI differ in morphology compared to layer IV interneurons and provide inhibition to surrounding pyramidal cells by extending axons to layer I and contacting their distal apical dendrites (Ma et al., 2006), which is the defining feature of so-called Martinotti cells.

In the absence of somatostatin interneurons, the feedback inhibition is removed and graded signals can continue to increase in intensity causing epilepsy and perhaps sensory overload, a key symptom experienced by patients with autism where sensory stimuli, both sounds or touch, are overwhelming. A reduction in the number of somatostatin interneurons is seen in mice deficient for both MeCP2 and PTEN; patients with mutations in these same genes exhibit autism and epilepsy (Ito-Ishida et al., 2015; Vogt et al., 2015). Ito-Ishida and colleagues (2015) found the MeCP2-mutant mice, deficient for somatostatin interneurons, was the basis for their epilepsy whereas deletion of this protein from parvalbumin-expressing interneurons resulted in motor, sensory, memory, and social deficits. These data clearly indicate that somatostatin and parvalbumin interneurons have distinct roles in cortical function. 


\section{5НТЗ receptor-expressing interneurons}

A third heterogeneous population of cortical interneurons express the serotonin receptor $5 \mathrm{HT} 3 \mathrm{a}$, they account for approximately $30 \%$ of the total population of interneurons (Morales et al., 1996; Morales and Bloom, 1997; Miyoshi et al., 2010; Vucurovic et al., 2010). They are found in all layers of the cortex, but a large proportion reside in upper layers (Karagiannis et al., 2009; Lee et al., 2010; Miyoshi et al., 2010). Within this population, Miyoshi and colleagues identified nine different subtypes and Lee and colleagues characterized a tenth subtype based on morphology, electrophysiological properties, and chemical markers (Lee et al., 2010; Miyoshi et al., 2010). Early studies suggested and later studies confirmed that the $5 \mathrm{HT} 3 \mathrm{aR}$-expressing populations of cortical interneurons label for chemical markers that include reelin, vaso-intestinal peptide (VIP), cholecystokinin (CCK), calretinin, and neuropeptide Y (NPY; Morales and Bloom, 1997; Lee et al., 2010; Miyoshi et al., 2010). Each of these groups is distinguished by morphological characteristics and electrophysiological properties. The electrophysiological properties of 5HT3aR interneurons are highly diverse, and include late-spiking, burst non-adapting, delayed non-fast spiking, irregular spiking, and intrinsic bursting interneurons (Lee et al., 2010; Miyoshi et al., 2010). Similarly, their morphologies differ ranging from interneurons that are neurogliaform in appearance with small cell bodies and short dendrites, to bipolar and multipolar interneurons with longer reaching dendrites and larger cell bodies (Lee et al., 2010; Miyoshi et al., 2010).

A few studies have linked deficits in these small populations to cortical disorders such as schizophrenia and autism, but their role in developmental neurological disease remains understudied (Hashimoto et al., 2008). Hashimoto and colleagues (2008) identified lower transcript levels of NPY and CCK in the dorsolateral prefrontal cortex in patients with schizophrenia implicating this group in neurological disease. However, further work is required to fully elucidate their role in other developmental disorders. 


\section{The Ventral Forebrain and Development of Cortical Interneuron Populations}

Each population of cortical interneuron arises from distinct regions of the ventral forebrain during development (Wichterle et al., 1999; Anderson et al., 2001; Nery et al., 2002; Xu et al., 2004; Miyoshi et al., 2010; Ma et al., 2012; Niquille et al., 2013). They migrate using guidance cues to navigate to the correct location in the cortex and to make the appropriate connections, some mentioned above, on excitatory neurons (Polleux et al., 2002; Flames et al., 2004; Zimmer et al., 2008; Bortone and Polleux, 2009; Elias et al., 2010; Rudolph et al., 2010; Sánchez-Alcañiz et al., 2011; Wang et al., 2011; Li et al., 2012; Abe et al., 2014).

The concept that neurons migrated within the developing cortex was first identified through the study of radially migrating neurons (Angevine and Sidman, 1961; Rakic, 1971, 1972). Studies determined that excitatory neurons are born in the ventricular zone from radial glia, migrate up radial glial scaffolding, and drop off in an inside-out manner to organize themselves based on birthdate (Angevine and Sidman, 1961; Rakic, 1974; Bayer et al., 1991; Noctor et al., 2001). Further, there are many important guidance cues and cytoskeletal regulators known to be essential for excitatory neuron migration, such as reelin and doublecortin (Stouffer et al., 2015). Perturbations of excitatory neuron migration result in a spectrum of human malformation syndromes including lissencephaly and the double cortex phenotype (Caviness, 1976; Gleeson et al., 1998, 1999). During subsequent studies of radially migrating excitatory neurons a second cohort of migrating neurons was identified (O'Rourke et al., 1995, 1997). Researchers later solidified that these neurons were GABAergic interneurons and that they migrated from regions in the ventral forebrain instead of the ventricular zone of the dorsal forebrain (Anderson et al., 1997; Wichterle et al., 1999, 2001). GABAergic neurons, including cortical interneurons, are generated in three anatomically distinct compartments within the developing ventral forebrain; the lateral ganglionic eminence (LGE), medial ganglionic eminence (MGE), and caudal ganglionic eminence (CGE; Lavdas et al., 1999; Wichterle et al., 1999; Anderson et al., 2001; 
Miyoshi et al., 2010). These regions are well-defined morphologically and by their transcription factor expression (Xu et al., 2004, 2008; Cai et al., 2013). Initial studies demonstrated that the MGE but not the LGE produced cortical interneurons that would disperse into adult cortical tissue suggesting that the LGE produced a different population of inhibitory neurons that did not migrate into the cortex (Wichterle et al., 1999). Further studies found that the lateral ganglionic eminence gives rise to inhibitory neurons in the striatum and olfactory bulb (Campbell et al., 1995; Wichterle et al., 1999; Anderson et al., 2001; Stenman et al., 2003; Tucker et al., 2006; Waclaw et al., 2009). Cortical interneurons arise predominately from the MGE and the CGE (Figure 1.1A,B; Wichterle et al., 1999, 2001; Xu et al., 2004; Lee et al., 2010; Miyoshi et al., 2010; Touzot et al., 2016). The MGE gives rise to the largest population of cortical interneurons including the parvalbumin- and somatostatin-expressing interneurons from the ventral and dorsal MGE respectively (Anderson et al., 2001; Xu et al., 2004; Inan et al., 2012). The CGE gives rise to most $5 \mathrm{HT} 3 \mathrm{aR}$ expressing interneurons including the VIP, CCK, and a subset NPY interneurons (Nery et al., 2002; Xu et al., 2004; Miyoshi et al., 2010; Touzot et al., 2016). A portion of NPY neurons are somatostatin-positive and are generated in the MGE (Butt et al., 2005).

\section{Cortical Interneuron Migration}

Cortical interneurons migrate tangentially from the MGE and CGE to reach the cortical rudiment and infiltrate the cortical plate (Figure 1.1C,E; Xu et al., 2004; Miyoshi et al., 2010; Inan et al., 2012). The birthplace of inhibitory cortical interneurons is therefore distinct from their excitatory neuron counterparts in that excitatory neurons are born in the ventricular zone of the dorsal forebrain and travel radially to enter the cortical plate on glial scaffolding (Rakic, 1972; Noctor et al., 2001). Excitatory neurons then drop off radial glial processes to form layers in the cortical plate and do so based on birthdate in an inside-out manner, with older neurons being deposited in deeper cortical layers (Angevine and Sidman, 1961; Rakic, 1974; Noctor et al., 2001; Greig et 
al., 2013). Interneurons migrating tangentially will later distribute together with excitatory neurons in the cortical plate according to birthdate. Early born interneurons from the MGE tend to co-distribute with early born excitatory neurons (Valcanis and Tan, 2003). Conversely, CGE derived inhibitory interneurons laminate primarily in upper layers of the cortex, not by birthdate like MGE derived interneurons (Miyoshi et al., 2010).

Cortical interneurons enter the cerebral cortex beginning on embryonic day 12.5 in mice (in humans this begins between 6-8 weeks of embryonic development; Figure 1.1D; Zecevic et al., 2011; Ma et al., 2013). Cortical interneurons mainly travel in two streams, a marginal zone (MZ) stream located just below the pial surface of the cortex, and a subventricular zone stream (SVZ) located above the ventricular zone (Figure 1.1C; Wichterle et al., 2001).

Migrating cortical interneurons travel similarly to radially migrating cells by extending a dynamic leading process (Figure 1.2A) that in conjunction with nuclear kinetic events pulls them forward (Figure 1.2B) in a process called nucleokinesis (Rakic, 1971; Edmondson and Hatten, 1987; Bellion et al., 2005). They differ from excitatory neurons in the frequency in which their leading process branches and the rapidity in which they change directions during migration (Nadarajah et al., 2001; Martini et al., 2009; Lysko et al., 2011), which likely reflects different demands associated with accomplishing guided migration without a radial glial substrate. Each of these elaborate events correlates with the expression of receptors and guidance cues or chemokines (Serrati et al., 2008; Sánchez-Alcañiz et al., 2011; Wang et al., 2011; Baudoin et al., 2012; Lysko et al., 2014).

\section{Migration Guided by Molecular Cues}

Guidance cues act as directional maps providing boundaries for migrating neurons within the developing forebrain (Flames et al., 2004; Rudolph et al., 2010; Sánchez-Alcañiz et al., 2011; Wang et al., 2011; Li et al., 2012). Without these maps, migration can go awry causing 
devastating cortical dysfunction (Meechan et al., 2012; Volk et al., 2015). Recent studies have found that cortical interneurons can express different receptors based on age, region of the forebrain, and which stream they are migrating (Faux et al., 2010; Antypa et al., 2011). Cortical interneuron guidance cues can be chemoattractive, guiding cortical interneurons through a region, or chemorepulsive, deterring cortical interneurons from an area (Flames et al., 2004; Rudolph et al., 2010; Sánchez-Alcañiz et al., 2011; Wang et al., 2011; Li et al., 2012). Cortical interneurons rely heavily on guidance cues to maintain streams and migrate to the appropriate location within the cerebral cortex (Flames et al., 2004; Rudolph et al., 2010; Sánchez-Alcañiz et al., 2011; Wang et al., 2011; Li et al., 2012).

\section{Early migration in streams}

Chemoattractive cues such as stromal derived factor-1 (SDF-1; also known as Cxcl12) provide preferential areas where cortical interneurons expressing the Cxcr4 receptor can migrate and distribute into their two main migratory streams (López-Bendito et al., 2008). The SDF-1 ligand is secreted into the extracellular environment by meningeal cells at the pial surface and transiently-amplifying neural progenitor cells in the SVZ (López-Bendito et al., 2008). The SDF-1 signaling pathway also mediates microtubule dynamics in the leading processes of cortical interneurons and affects the speed of migration and decision making during migration (Lysko et al., 2011). The absence of this cue causes dispersion of cortical interneuron streams and disruption to their placement and wiring with other neurons within the cortex (Sánchez-Alcañiz et al., 2011; Wang et al., 2011). One example where Cxcr4 expression is altered within cortical interneurons is in the DiGeorge Syndrome, or 22q.11 deletion syndrome, which is often associated neurological dysfunction including schizophrenia and autism (Meechan et al., 2012). Meechan and colleagues described the distribution of cortical interneurons in the Large Deletion (LgDel) 22q11.2DS mouse model of DiGeorge Syndrome to be similar to Cxcr4 mutants. The LgDel model showed a decrease of cortical interneurons traveling within the SVZ stream and an 
increase in interneurons in the $\mathrm{MZ}$ stream which is comparable to cortical interneuron distributions seen in Cxcr4 null mice (Wang et al., 2011; Meechan et al., 2012). Moreover, the distribution shifts seen in early cortical development do affect the distribution of parvalbumin interneurons and their circuitry in the adult brain (Meechan et al., 2012, 2015). Dysfunctional wiring of parvalbumin-positive interneurons was associated with cognitive impairment in the LgDel mouse (Meechan et al., 2015). This was assessed with a visual discrimination/reversal task and demonstrated that the LgDel mice had difficulty learning the initial task and further, had difficulty when the task was reversed (Meechan et al., 2015). These data indicate that the proper guidance of cortical interneurons by Cxcr4 is imperative to for proper function of the adult cortex.

On the other hand, chemorepellents like ephrins repel cortical interneurons from inappropriately invading the ventral forebrain and cortex. Two types of ephrin ligands exist: Type A and Type B, distinguished by their interactions with EphA versus EphB receptors and the fact that the type A's are glycosyl-phosphatidylinositol linked while the type B's are transmembrane (Gale et al., 1996; Flanagan and Vanderhaeghen, 1998). The ephrins thought to guide interneuron migration are mostly ephrin A's (Zimmer et al., 2008; Rudolph et al., 2010). There are several different isoforms of ephrin A's that are expressed in different locations of the developing forebrain. However, studies of ephrin guidance of cortical interneuron migration remain confined to the ventral forebrain. Ephrin A5, expressed in the VZ of both the MGE and LGE, acts to repel cortical interneurons expressing its cognate receptor, EphA4, arising and migrating from the MGE (Zimmer et al., 2008). A stripe assay utilizing cortical interneurons found that interneurons did not enter the stripes of ephrin A5. Additionally, cocultures of MGE derived cortical interneurons and fibroblasts transfected to express ephrin A5 demonstrated that ephrin A5 repels cortical interneurons away from the transfected fibroblasts. Further, ephrin A3 also interacts with EphA4 receptors to guide cortical interneurons within the developing cortex 
(Rudolph et al., 2010). The ephrin A3 ligand was found to be expressed in the cortical plate and developing striatum (Rudolph et al., 2010). Slice culture assays inhibiting ephrin A3 made the striatum permissive to cortical interneurons migrating from the MGE and stripe assays demonstrated that ephrin A3 repels cortical interneurons. (Rudolph et al., 2010). However, the involvement of ephrin A3 in the cortex remains unknown. If ephrin A3 signaling acts similarly in the cortical plate to its repellent role in the striatum it is possible that the expression of EphA4 receptors would prevent the entry of cortical interneurons into the cortical plate and promote tangential migration in streams.

There are some guidance cues that affect cortical interneuron migration where it is still unclear whether the guidance cue is chemoattractive or repulsive. Cortical interneurons guided by ErbB4 receptors in response to the neuregulin ligand is one example (Flames et al., 2004; Li et al., 2012). Disruptions to ErbB4 signaling are implicated in cases of schizophrenia (Stefansson et al., 2002). ErbB4 receptors are primarily localized on cortical interneurons migrating from the MGE and in the adult cortex, particularly on parvalbumin- expressing neurons (Yau et al., 2003). ErbB4 is activated specifically by neuregulin 1 and 3 during early brain development (Flames et al., 2004; Li et al., 2012). Li and colleagues (2012) demonstrated that neuregulin 1 and 3 are expressed in different regions of the developing cortex; neuregulin 3 is expressed within the developing cortical plate, and neuregulin 1 is expressed in VZ/SVZ at E14.5. Neuregulin 1 has three distinct isoforms all of which regulate cortical interneuron migration (Flames et al., 2004; Li et al., 2012). The secreted isoforms (neuregulin 1 type I and II) were found to be chemoattractive to migrating cortical interneurons (Flames et al., 2004). The membrane bound isoform (neuregulin 1 type III) created an environment that was permissive for migrating interneurons (Flames et al., 2004). However, it remains uncertain whether neuregulins act as a chemoattractant or a chemorepellent to guide cortical interneuron migration during early development (Flames et al., 2004; Li et al., 2012). Flames and colleagues (2004) suggest that 
neuregulins attracts migrating neurons. In vitro stripe assays, using dissociated MGE cells and COS cells transfected with neuregulin 1 type III, demonstrated that cortical interneurons migrated towards neuregulin 1 type III expressing COS cells and preferred them over control or nontransfected COS cells (Flames et al., 2004). Similarly, in vitro assays utilizing MGE explants grown in culture with neuregulin 1 type I transfected COS cells showed a similar result (Flames et al., 2004). However, a more recent study found neuregulin acts as a chemorepellent and instead of attracting cortical interneurons suggested that neuregulins act to create boundaries through which cortical interneuron streams can travel (Li et al., 2012). In vitro MGE explant assays show that when MGE explants are cultured with T293 cells transfected with neuregulin 1 and 3, explants showed polarized growth away from transfected T293 cells (Li et al., 2012). Further, in-utero electroporation of neuregulin 1 within the developing cortex prevents cortical interneuron migration into the area of ectopic expression (Li et al., 2012). Therefore, although neuregulins are important for early cortical interneuron migration their primary role as a guidance factor remains unclear.

\section{Tangential to radial transition}

As development advances migrating cortical interneurons need to exit from the cohesive streams they are traveling in to invade the cortical plate and establish residence in the appropriate laminae. This requires tight regulation of chemokine cues and also expression of new cues both in the interneuron as well as in the extracellular environment. Each step in development must proceed without error to establish normally functioning cortex.

Around E15.5 cortical interneurons make a crucial transition from tangential migration to radial migration to infiltrate the developing cortical plate. Premature transitions or failure to make this transition can result in improper positioning of interneurons in the cortex (Sánchez-Alcañiz et al., 2011; Wang et al., 2011; Meechan et al., 2012). Initial studies on this transition suggested that 
there is specific regulation of guidance factors which range from regulation of receptors that read chemokine guidance cues (Cxcr4 and Cxcr7) to the expression of adhesion molecules (Connexin43) that mediate the tangential to radial transition (Elias et al., 2010; Sánchez-Alcañiz et al., 2011; Wang et al., 2011; Abe et al., 2014).

The SDF-1 pathway is known for perpetuating a tangential orientation in cortical interneurons through Cxcr4 and Cxcr7 (Sánchez-Alcañiz et al., 2011; Wang et al., 2011; Abe et al., 2014). Elimination of either $\mathrm{Cxcr} 4$ or 7 causes cortical interneurons to disperse from migratory streams and alter positioning of interneurons within the mature cortex, indicating that both receptors are important for spatial and temporal guidance (Sánchez-Alcañiz et al., 2011; Wang et al., 2011). However, studies have shown that these receptors may have distinct roles during cortical interneuron migration, specifically relating to their tangential to radial transition (Lysko et al., 2011, 2014; Sánchez-Alcañiz et al., 2011; Wang et al., 2011; Abe et al., 2014).

Recent studies suggest that $\mathrm{Cxcr7}$ may have both autonomous and non-autonomous roles in cortical interneurons migration and is important for regulating the expression Cxcr4 and SDF-1 (Sánchez-Alcañiz et al., 2011; Wang et al., 2011). During early cortical development at E13.5 in mice, cortical interneurons primarily migrate in two distinct streams highly regulated by Cxcr4 expression and the presence of the SDF-1 ligand (Sánchez-Alcañiz et al., 2011; Wang et al., 2011). Cxcr7 promotes the retention of Cxcr4 at the cell's surface (Sánchez-Alcañiz et al., 2011; Abe et al., 2014). Additionally, at E13.5, Cxcr7 is highly expressed within the cortical plate by excitatory neurons (Sánchez-Alcañiz et al., 2011). In vitro studies using somatic cell culture demonstrated that Cxcr7 was expressed on both a cell's surface and internally, whereas Cxcr4 was expressed only on the cell's surface (Boldajipour et al., 2008). Cxcr7 was capable of binding and sequestering SDF-1 internally within the cell (Boldajipour et al., 2008). It is possible that Cxcr7 plays a non-autonomous role during early stages of cortical interneuron migration by acting within excitatory neurons to sequester SDF-1 in the cortical plate to localize cortical 
interneuron to streams and prevent cortical interneurons from entering the cortex (Boldajipour et al., 2008; Sánchez-Alcañiz et al., 2011). During later time points the expression of Cxcr7 is down-regulated in the cortical plate, perhaps allowing the cortical plate to be permissive to cortical interneurons potentially through SDF-1 signaling (Sánchez-Alcañiz et al., 2011). Further, the expression of SDF-1 affects the stabilization of microtubule networks within the leading process of cortical interneurons through the phosphorylation of Dcx by protein kinase A (PKA; Lysko et al., 2011, 2014). In the presence of PKA, Dcx binds to and stabilizes microtubules which in turn prevents branching of the leading process (Lysko et al., 2014). In the absence of SDF-1 activation of Cxcr4, microtubule networks destabilize and leading process branching occurs (Lysko et al., 2011). Leading process branching could be one way cortical interneurons turn radially to enter and navigate the cortical plate. This, in conjunction with $\mathrm{Cxcr7}$ regulation of Cxcr4 at the surface of migrating interneurons, could indicate that Cxcr7 is important for organization of cortical interneurons within streams during early development and also their departure to enter the cortical plate during later development.

However, regulation of the SDF-1 pathway is unlikely to be the only regulator of the tangential to radial switch. Recently, studies began to investigate this further and documented differences in the expression of receptors or cues that mediate changes within the migrating interneuron, as well as changes in the expression of environmental cues that occur during this time period that could all work together to promote proper placement in the cortical plate (Bortone and Polleux, 2009; Elias et al., 2010; Baudoin et al., 2012; Lin-Hendel et al., 2016).

Elias and colleagues (2010) were the first to report that both radially migrating excitatory neurons and interneurons use gap junctions to migrate. Connexins are gap junction proteins that create channels to communicate electrical and chemical signals between neurons. During development, excitatory neurons express Connexins and utilize them as cell adhesion molecules, independent of their ability to form pores between cells, to adhere to radial glial cells 
during their migration into the cortical plate (Elias et al., 2007). Similarly, they found that expression of Connexin43, a gap junction protein in cortical interneurons, is important for initiating the radial transition (Elias et al., 2010). When Connexin43 was knocked down in interneurons few made a radial transition to travel into the cortical plate (Elias et al., 2010). Electron microscopy showed that radially migrating cortical interneurons can establish connections via Connexin43 with radial glia to migrate into the developing cortical plate in the same way as excitatory neurons (Elias et al., 2010). These studies demonstrate that cortical interneurons may use similar mechanisms as migrating excitatory neurons when contacting and migrating on radial glia. However, it still remains unclear what mechanism provides the onswitch for the expression of these adhesion proteins. Perhaps the expression of adhesion proteins arises following changes to the branching and orientation of the leading process.

Studies have indicated that the primary cilium, a highly specialized system of microtubules, acts to help cortical interneurons read and respond to guidance cues within their environment and orient cortical interneurons as they migrate (Baudoin et al., 2012; Higginbotham et al., 2012). Conditional deletion of the ciliary protein, Arl13b, caused defective ciliary dynamics within cortical interneurons which triggered, delayed migration into the developing cortex, and altered the radial distribution of cortical interneurons within the cortical wall (Higginbotham et al., 2012). In Arl13b mutants, more cortical interneurons were found in the IZ of the cortical wall, with a marked decrease of interneurons traveling in the MZ and SVZ streams (Higginbotham et al., 2012). Many of the cortical interneurons had increased branching compared to controls, and increased pausing (Higginbotham et al., 2012). Further, in vitro cortical interneuron cultures demonstrated that the expression and shuttling of guidance cue receptors were altered in the primary cilium, indicating that the altered distribution of cortical interneurons within the cortical wall may be due to their inability to appropriately process environmental guidance cues (Higginbotham et al., 2012). Additionally, Baudoin and colleagues (2012) found Sonic 
Hedgehog $(\mathrm{SHH})$ signaling through the primary cilium orients cortical interneurons during their migration. Depletion of SHH signaling with cyclopamine in ex-vivo slices caused cortical interneurons to remain in streams migrating tangentially (Baudoin et al., 2012). However, in the presence of $\mathrm{SHH}$, cortical interneurons departed from migratory streams and entered the cortical plate (Baudoin et al., 2012). Shuttling of the SHH pathway proteins through intraflagellar transport (IFT) was found to be required for cortical interneurons to make the transition to a radial orientation (Baudoin et al., 2012). Disruption to anterograde IFT, by conditionally ablating Kif3a from cortical interneurons, showed that cortical interneurons in ex vivo slices could not respond to SHH signaling and were stuck in a persistent tangential orientation (Baudoin et al., 2012). Therefore, appropriate assembly and function of the primary cilium is necessary for cortical interneurons to respond to guidance cues and could play an important role when cortical interneurons make the tangential to radial transition.

Moreover, energy utilization may also assist with the tangential to radial transition during this time. Lin-Hendel and colleagues (2016) demonstrated the need for oxidative phosphorylation for tangentially migrating cortical interneurons that was not required for radial projection neurons migration. Deletion of Ant1, a transporter important for transferring ATP across the inner membrane of mitochondria, in mice resulted in cortical interneurons changing directions instead of maintaining their tangential orientation (Lin-Hendel et al., 2016).

In summary, these studies suggest that multiple molecular mechanisms work synergistically to regulate the appropriate timing of cortical interneuron departure from migratory streams. The manner in which these distinct molecular mechanisms are coordinated during interneuron migration is unknown, but intracellular signaling pathways may play a vital role in the process. Signaling pathways are known to modify movement and direction in neurons as well as other migratory cell types through phosphorylation of downstream targets, but their role in early cortical interneuron migration remains incompletely understood. 


\section{The c-Jun N-Terminal Kinase Signaling Pathway}

The c-Jun N-terminal kinase (JNK) signaling pathway consists of members of the mitogenactivated protein kinase (MAPK) superfamily of protein kinases. Three genes, JNK1, JNK2, and JNK3, encode the terminal kinases of the JNK signaling pathway (Gupta et al., 1996). The family of three $J N K$ genes produce a total of ten isoforms through alternative splicing that exist in a short form $(46 \mathrm{kDa})$ or a long form $(55 \mathrm{kDa}$; Gupta et al., 1996). Each JNK protein is capable of activating different substrates and downstream cascades through phosphorylation of serine and threonine residues. In addition, JNK-interacting proteins (JIPs) assist JNKs by providing a scaffolding that bind JNKs to either increase the probability of being phosphorylated by an upstream effector or to phosphorylate a downstream target (Whitmarsh and Davis, 1998; Whitmarsh et al., 1998).

The JNK pathway has traditionally been associated with its role in apoptosis through its phosphorylation of c-Jun, a downstream activator of the cell death cascade across many cell types (Kuan et al., 1999) . However, JNK is important for a multitude of other functions including axon guidance and growth in neurons and cytoskeletal rearrangement. It is important for migration in a variety of cell types including migration of excitatory neurons during brain development (Petrich et al., 2002; Chang et al., 2003; Gdalyahu et al., 2004; Kim and Sharma, 2004; Oliva Jr et al., 2006; Jin et al., 2010; Westerlund et al., 2011).

\section{Brain development and neuronal migration}

Early studies implicated JNK signaling in nervous system development and suggest differential roles for each Jnk gene in different locations within the brain (Kuan et al., 1999). Kuan and colleagues determined a clear onset of expression of JNK within the developing brain beginning at embryonic day 7 for Jnk1 and Jnk2 and embryonic day 11 for Jnk3 (Kuan et al., 1999). Additionally, they found distinct expression patterns throughout the developing embryo (Kuan et 
al., 1999). Initial studies of single deletions did not reveal a neurological phenotype in mice (Kuan et al., 1999). However, combinatorial constitutive deletion of the Jnk1 and Jnk2 genes were found to cause embryonic lethality between embryonic day 11 and 12 in mice (Kuan et al., 1999). The Jnk1/2 knockout mice displayed neural tube closure defects and decreased apoptosis in the hindbrain, in addition to increased apoptosis in the forebrain (Kuan et al., 1999). Combinatorial deletions of Jnk1/3 and Jnk2/3 did not show similar anomalies (Kuan et al., 1999).

In adults, JNK proteins from all three JNK genes were found to be highly expressed within the cortex, hippocampus, striatum, and cerebellum of wildtype mice (Brecht et al., 2005). The short isoforms of Jnk1 and Jnk3 were predominately expressed over the long isoforms, whereas both short and long isoforms of Jnk2 were expressed equally. Jnk1 expression was primarily through the short isoform in all regions (cortex, hippocampus, striatum, and cerebellum; Brecht et al., 2005).

More recent studies demonstrate potential need for all three JNK genes in developing cortex. Single loss of the Jnk1 or Jnk2 gene in mice are now known to be sufficient to disrupt excitatory neuron migration in the developing cerebral cortex. In vivo studies by Westerlund and colleagues revealed that $J n k 1$ is important for excitatory neuron migration and in its absence, causes them to migrate faster into the cortical plate (Westerlund et al., 2011). Additional studies established that excitatory neurons exhibiting a loss of JNK displayed dysfunction during nucleokinesis, and migration defects (Nishimura et al., 2010, 2014). Recently, Zhang and colleagues (2016) demonstrated a need for Jnk2 in migrating excitatory neurons. The absence of Jnk2 caused delays in migrating excitatory neurons, suggesting different roles for the Jnk genes within migrating neurons (Zhang et al., 2016). 
Human mutations in JNK genes are not well documented. Interestingly, the only documented mutations are located in the JNK3 gene (Shoichet et al., 2006; Kunde et al., 2013). Truncation mutations of the JNK3 gene cause cognitive deficits and epilepsy (Shoichet et al., 2006; Kunde et al., 2013) suggesting that dysfunctions in the JNK genes could underlie certain cortical disorders. Other more obscure disruptions to the JNK pathway exist and occur through mutations in other genes that either activate the JNK pathway, or function downstream of the JNK pathway, and are a plausible cause of neurological disorders. One such regulator of JNK signaling that is found mutated in autism spectrum disorder is TAOK2, which is an upstream activator of JNK function (Calderon de Anda et al., 2012). Although, there is very little evidence to show this interaction, mouse models suggest that these mutations decrease activation of the JNK pathway and subsequently cause defects in developing neurons (Calderon de Anda et al., 2012).

\section{Neuronal motility and movement}

Several downstream effectors of JNK signaling regulate cell motility and movement during migration and are known to cause neurological dysfunction when disrupted. Stathmin 10 and MARKS-like protein 1 proteins are important for regulating cytoskeletal elements within excitatory neurons (Westerlund et al., 2011; Björkblom et al., 2012). These proteins bind to microtubules and actin filaments, respectively, to regulate branching and neuronal movement. Likewise, doublecortin (Dcx), a microtubule-associating protein, is also a phosphorylation target of JNK signaling (Gdalyahu et al., 2004; Jin et al., 2010). In humans, disruption of DCX causes severe cortical migration disorders such as double cortex syndrome (Gleeson et al., 1998). Disruption of doublecortin in migrating cortical interneurons decreases branch formation and nucleokinesis events in cortical interneurons making it a likely contributor to other disorders of the cortex (Kappeler et al., 2006). JNK-phosphorylation of doublecortin causes it to localize to growth cones of cultured hippocampal neurons, which perhaps allows the growing axon to 
perform different functional movements (Gdalyahu et al., 2004; Lysko et al., 2014; Nishimura et al., 2014). This localization of doublecortin was absent from the growth cone in the presence of a pan-JNK inhibitor (Gdalyahu et al., 2004).

These studies suggest an internal role for JNK in migrating neurons that affect not only the cytoskeletal structure of the neuron, but also its ability to respond to external cues in the environment. It is still not known how JNK signaling activity differs amongst different subsets of neurons.

\section{Objectives and Hypotheses}

The JNK signaling pathway plays a crucial role in migrating neurons, specifically excitatory neurons in the developing cortex. Studies from Westerlund and colleagues and Zhang and colleagues show that there are differing, but important roles, for individual Jnk genes in migrating excitatory neurons, which can modify the speed that they migrate within the cortex and ultimately affect how they are deposited into distinct layers (Westerlund et al., 2011; Zhang et al., 2016). Truncations of JNK genes in humans suggest that cortical interneurons could be disrupted by altered JNK function, as patients present with epilepsy and intellectual disability; cortical diseases that are associated with deficits in inhibition (Shoichet et al., 2006; Kunde et al., 2013). However, the role that JNK signaling plays in migrating cortical interneurons is not known.

Thus, JNK signaling is likely to play important roles in cortical interneuron development and understanding JNK's role may assist in understanding the pathogenesis of several human neurodevelopmental disorders. Therefore, the primary objective of this research is to characterize the role that JNK signaling plays in early cortical interneuron migration. The first set of experiments in Chapter 2 of my thesis explores the role of JNK signaling during the early entry of cortical interneurons into the cerebral cortex at embryonic day 12.5 . The second set of 
experiments presented in Chapter 3 of my thesis delves into the role that JNK signaling plays in migratory stream integrity and interneuron laminar positioning in the cortical plate. Our overarching central hypothesis for this work is that JNK signaling plays a crucial role during the early migration of cortical interneurons as they first enter and then find their laminar position in the developing cerebral cortex. 


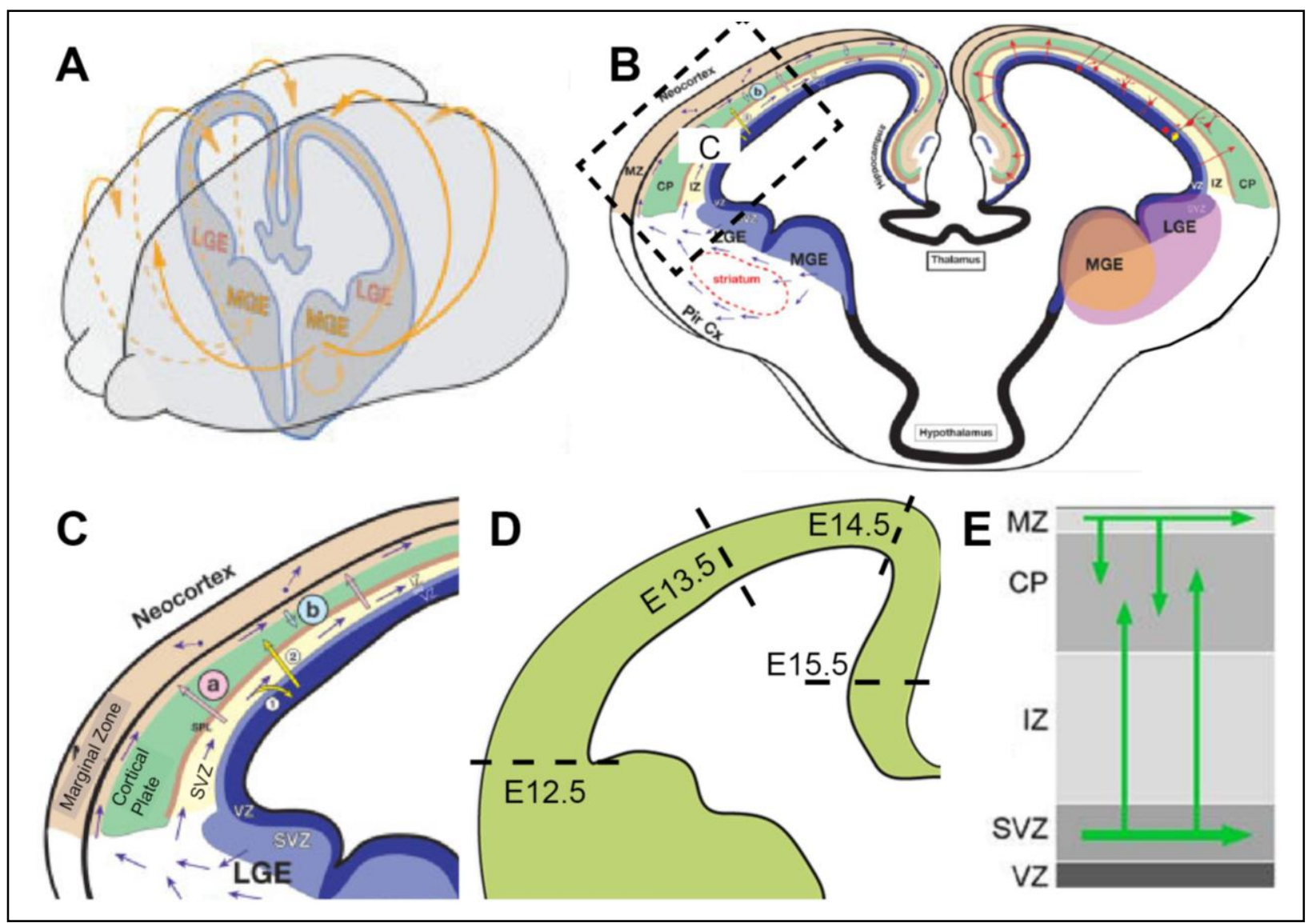

Figure 1.1: Cortical interneuron migration. A. Cortical interneurons migrate from distinct regions in the embryonic mouse brain. B. A coronal section of an embryonic mouse brain. C. Cortical interneurons migrate tangentially from the ventral forebrain in two streams located in the marginal zone (MZ) and subventricular zone (SVZ). D. Cortical interneurons arrive at different portions of the cortex at different time points ( $E=$ embryonic day of mouse development). E. In order to enter the cortical plate cortical interneurons turn to migrate radially ( $\mathrm{MZ}=\mathrm{Marginal}$ zone, $\mathrm{CP}=$ Cortical plate, $\mathrm{IZ}=$ Intermediate zone, SVZ=Subventricular zone, VZ=Ventricular zone). Figure includes adapted figures from: (Danglot et al., 2006; López-Bendito et al., 2008; Marín et al., 2010) 


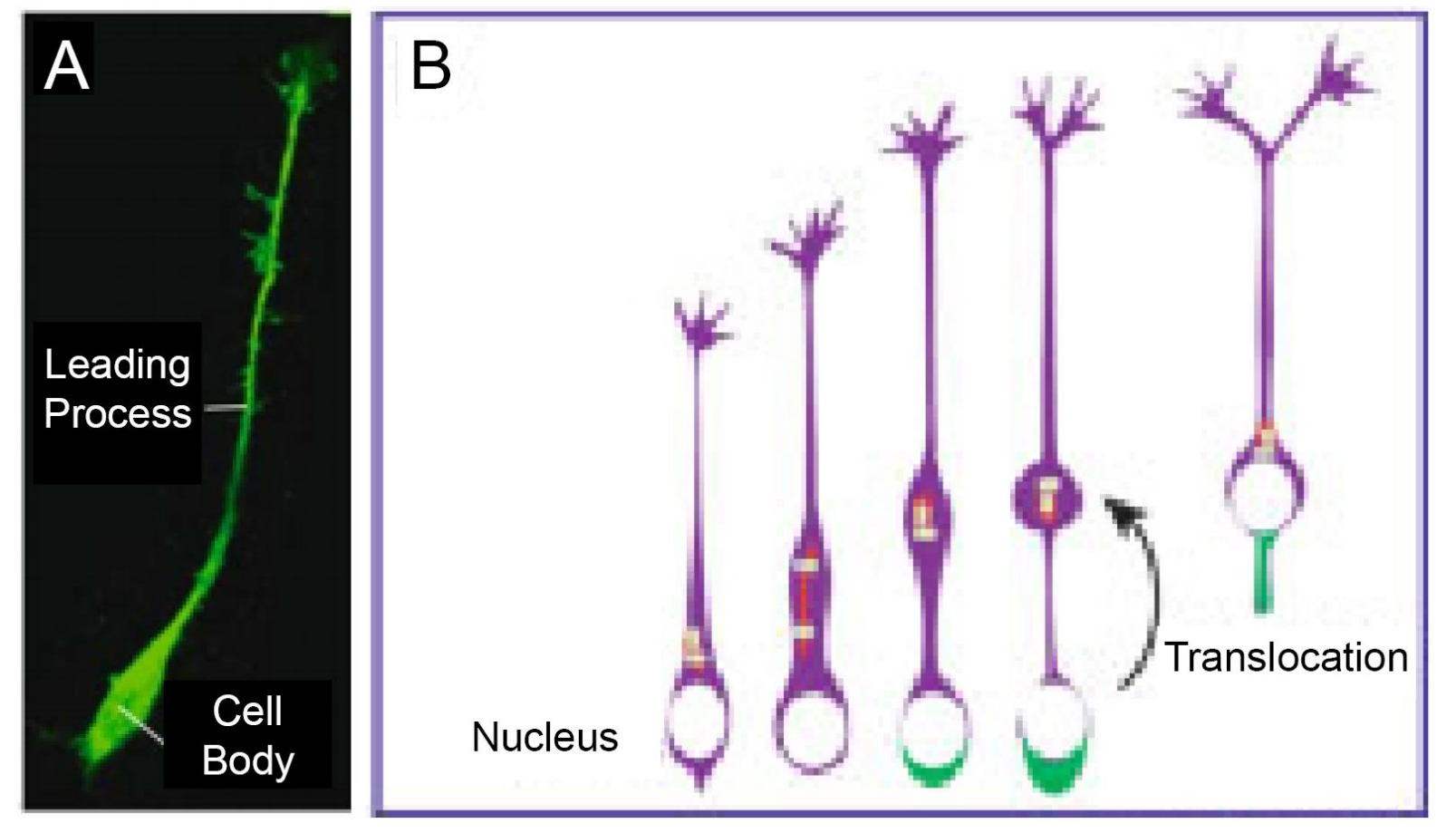

Figure 1.2: Migrating cortical interneurons. A. Migrating cortical interneurons extend a leading process that helps them to navigate their way into the developing cerebral cortex. B. Migrating cortical interneurons create a swelling in the leading process that is equipped with the cellular machinery to pull the cell body forward. Figure includes adapted figures from: (Polleux and Anton, 2005; Danglot et al., 2006) 


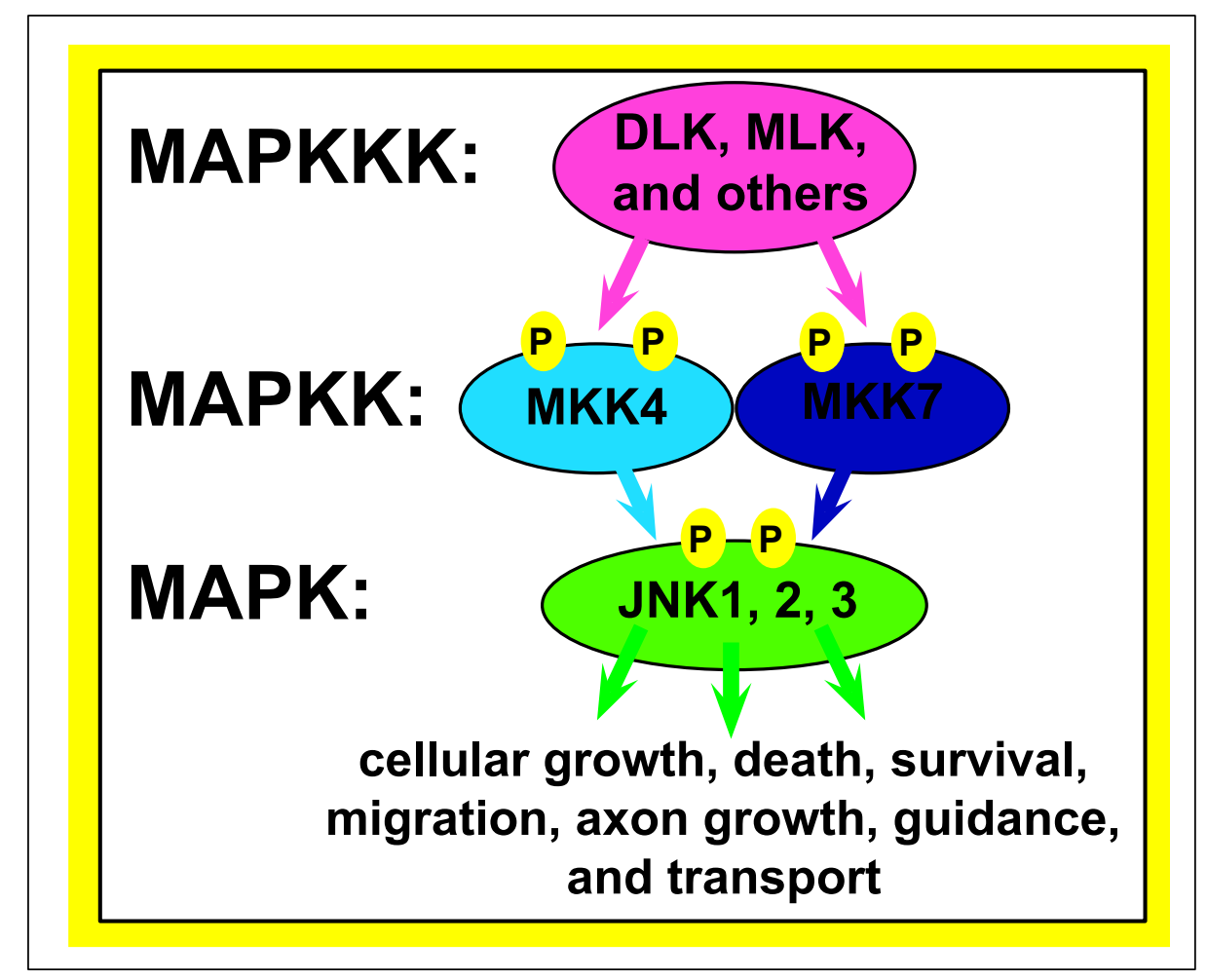

Figure 1.3: The c-Jun-N-terminal kinase (JNK) pathway. JNK is phosphorylated by multiple upstream kinases. JNK is the terminal kinase in this cascade activating multiple downstream targets to regulate many cell growth and migration activities. 


\title{
Chapter 2: Cortical Interneurons Require Jnk1 to Enter and Navigate the Developing Cerebral Cortex
}

\author{
Abigail K Myers ${ }^{1,2,3}$, Daniel Meechan ${ }^{4}$, Danielle R Adney ${ }^{1,3}$, and Eric S Tucker ${ }^{1,3}$ \\ ${ }^{1}$ Department of Neurobiology and Anatomy, ${ }^{2}$ Neuroscience Graduate Program, ${ }^{3}$ Center for \\ Neuroscience, West Virginia Univ. Sch. of Med., Morgantown, WV 26506 \\ ${ }^{4}$ Department of Pharmacology and Physiology, The George Washington Univ. Med. Ctr., \\ Washington, DC 20037
}

Published in the Journal of Neuroscience, June $4^{\text {th }}, 2014$ 


\section{Abstract}

Proper assembly of cortical circuitry relies on the correct migration of cortical interneurons from their place of birth in the ganglionic eminences to their place of terminal differentiation in the cerebral cortex. Although molecular mechanisms mediating cortical interneuron migration have been well studied, intracellular signals directing their migration are largely unknown. Here we illustrate a novel and essential role for c-Jun $\mathrm{N}$-terminal kinase (JNK) signaling in guiding the pioneering population of cortical interneurons into the mouse cerebral cortex. Migrating cortical interneurons express Jnk proteins at the entrance to the cortical rudiment and have enriched expression of Jnk1 relative to non-interneuronal cortical cells. Pharmacological blockade of JNK signaling in ex vivo slice cultures resulted in dose-dependent and highly specific disruption of interneuron migration into the nascent cortex. Time-lapse imaging revealed that JNK-inhibited cortical interneurons advanced slowly and assumed aberrant migratory trajectories while traversing the cortical entry zone. In vivo analyses of JNK-deficient embryos supported our ex vivo pharmacological data. Deficits in interneuron migration were observed in Jnk1 but not Jnk2 single nulls, and those migratory deficits were further exacerbated when homozygous loss of Jnk1 was combined with heterozygous reduction of Jnk2. Finally, genetic ablation of Jnk1 and Jnk2 from cortical interneurons significantly perturbed migration in vivo, but not in vitro, suggesting JNK activity functions to direct their guidance rather than enhance their motility. These data suggest JNK signaling, predominantly mediated by interneuron expressed Jnk1, is required for guiding migration of cortical interneurons into and within the developing cerebral cortex. 


\section{Introduction}

Cortical interneurons play vital roles regulating neurotransmission in the cerebral cortex and their dysfunction is implicated in severe brain disorders including epilepsy and schizophrenia. Mechanisms contributing to the pathological vulnerability of cortical interneurons are unclear, but they may arise during embryonic development when interneurons embark on long distance tangential migration from the medial and caudal ganglionic eminences to the overlying cerebral cortex (Anderson et al., 1997; Lavdas et al., 1999; Sussel et al., 1999; Wichterle et al., 2001; Nery et al., 2002; Miyoshi et al., 2010). For this reason, molecular mechanisms guiding cortical interneuron migration have been extensively studied (For review, see Faux et al., 2012; Marín, 2013). Many ligands and receptors mediating cortical interneuron dispersion from the ventral forebrain (Powell et al., 2001), motility (Polleux et al., 2002; Pozas and lbáñez, 2005), chemorepulsion from the striatum (Marín et al., 2001), chemoattraction to the cerebral cortex (Yau et al., 2003; Flames et al., 2004), and formation and maintenance of migratory streams have been identified (Tiveron et al., 2006; Li et al., 2008; LópezBendito et al., 2008; Sánchez-Alcañiz et al., 2011; Wang et al., 2011). However, intracellular signals controlling cortical interneuron migration, particularly those directing migration at critical positions along their trajectories, are largely unknown.

The c-Jun N-terminal protein kinase (JNK) pathway plays obligate roles in mammalian forebrain development (Kuan et al., 1999), and moreover, genetic disruption of JNK function leads to cognitive disorders in humans (Kunde et al., 2013). JNKs are members of the mitogen-activated protein kinase (MAPK) signaling pathway, and are encoded by three related genes in mammals: Jnk1 (Mapk8), Jnk2 (Mapk9), and Jnk3 (Mapk10). All three Jnk genes are expressed in the developing mouse brain, but only combinatorial deletion of Jnk1 and Jnk2 results in embryonic lethality and profound alterations in 
neuronal survival and programmed cell death (Kuan et al., 1999; Sabapathy et al., 1999). Genetic deletions of Jnk1 or upstream Jnk kinases results in aberrant radial migration of cortical projection neurons (Hirai et al., 2006; Wang et al., 2007; Westerlund et al., 2011; Yamasaki et al., 2011), strongly implicating JNK signaling as a major regulator of neuronal migration in the developing forebrain.

In the current study, we use a combination of ex vivo and in vivo approaches to demonstrate that cortical interneurons have a cell-intrinsic requirement for JNK signaling —largely mediated by the activity of Jnk1-to enter and successfully navigate the developing cerebral cortex. JNK-inhibited cortical interneurons dramatically slow their advancement and take inappropriate trajectories at the entrance to the cortical rudiment. Similarly, cortical interneurons in Jnk1-deficient and Jnk1/2-deficent embryos exhibit significant delays in their cortical migration. Conditional ablation of Jnk1 and Jnk2 from cortical interneurons delays cortical entry, disrupts migratory streams, and perturbs interneuron morphology in vivo, but has no effect in vitro, suggesting JNK signaling only regulates migration of cortical interneurons in the presence of cortical guidance cues. These findings implicate the JNK pathway, and Jnk1 in particular, as a key molecular node in the intracellular regulation of cortical interneuron migration in vivo. 


\section{Materials and Methods}

\section{$\underline{\text { Animals }}$}

Animals were housed and cared for by the Office of Laboratory Animal Resources at West Virginia University. Timed-pregnant dams (day of vaginal plug = embryonic day $0.5)$ were killed by rapid cervical dislocation and mouse embryos were immediately harvested for tissue culture, gene expression, or histological analyses. The following mouse strains were acquired and maintained on a C57BL/6J (The Jackson Laboratory) background: DIx5/6 Cre-IRES-EGFP (DIx5/6-CIE; Stenman et al., 2003), Mapk $8^{\text {tm1Rjd }}$ floxed (Jnk1fl/fl; Das et al., 2007; Kindly provided by Dr. Roger Davis), Mapk ${ }^{\text {tm1Flv }}$ knockout (Jnk1 ${ }^{-/}$; Dong et al., 1998; The Jackson Laboratory), and Mapk9 ${ }^{\text {tm1Flv }}$ knockout (Jnk2 ${ }^{-/}$; Yang et al., 1998; The Jackson Laboratory). For slice culture experiments, CF-1 (Charles River) dams were crossed with hemizygous DIx5/6-CIE males. Constitutive mutant embryos were generated by crossing Jnk $1^{+-} ; \mathrm{Jnk} 2^{+/-}$females to Jnk $1^{-/-}$or Jnk2/males. Conditional mutant embryos were generated by crossing Jnk $1^{f / f l /} ; J n k 2^{-/-}$females to DIX5/6-CIE; Jnk ${ }^{f l /+}$; Jnk2 ${ }^{-/}$males. All animal procedures were performed in accordance to protocols approved by the Institutional Animal Care and Use Committee at West Virginia University.

\section{Fluorescently Activated Cell Sorting and qRT-PCR}

Methods for fluorescently activated cell sorting (FACS), extracting RNA, synthesizing cDNA, and performing quantitative RT-PCR were reported previously (Meechan et al., 2012). Briefly, cortices from DIx5/6-CIE+ embryos were individually dissociated with papain (Worthington Biochemical), resuspended in FACS buffer, sorted based on endogenous GFP fluorescence, and DIx5/6-CIE(+) and DIx5/6-CIE(-) sorted samples were homogenized separately in Trizol reagent (Ambion). Total RNA was isolated and 
cDNA synthesized using the Improm-II RT kit (Promega). qPCR was conducted using EvaGreen (BioRad) reagent and a CFX384 thermal cycler (BioRad). PCR for Jnk1 (F: AGCAGAAGCAAACGTGACAAC /R: GCTGCACACACTATTCCTTGAG) and Jnk2 (F: CCAGTAGGATTGCCTGCTTA /R: TGGTCACATGCATACGAGTC) was performed. Gapdh was used as the endogenous reference gene control. Fold-change was determined by the delta-delta CT method (Livak and Schmittgen, 2001).

\section{Organotypic Slice Cultures}

Embryos were collected and dissected in ice-cold complete Hank's Balanced Salt Solution (cHBSS; Tucker et al., 2006). Dlx5/6-CIE+ brains were embedded in 3\% low melting point agarose (Fisher Scientific), sectioned at $300 \mu \mathrm{m}$ on a Leica VT1000 S vibratome, transferred to laminin/poly-D-lysine-coated transwell inserts (BD Falcon; Polleux and Ghosh, 2002) and grown at $37^{\circ} \mathrm{C}$ with $5 \% \mathrm{CO}_{2}$ in slice culture media (Tucker et al., 2006) for 24 hours. Stock solutions of pharmacological inhibitors [20 mM SP600125 (ENZO Life Sciences), 20 mM AS601245 (ENZO Life Sciences), and 100 mM U0126 (EMD Chemicals, Inc.)] were prepared in DMSO, stored at $-20^{\circ} \mathrm{C}$, and added to slice culture media immediately prior to use. DMSO was used as a vehicle control at matching solvent concentrations to inhibitors.

\section{Live Imaging Experiments}

Live vibratome slices were transferred to Millicell cell culture inserts (Millipore) in FluoroDishes (World Precision Instruments, Inc) filled with slice culture media containing a 1:1000 dilution of DMSO (for controls) or $20 \mu \mathrm{M}$ SP600125 JNK inhibitor. FluoroDish preparations were immediately transferred to a Zeiss LSM 510 Meta Confocal Microscope with stable environmental controls maintained at $37^{\circ} \mathrm{C}$ with $5 \%$ humidified 
$\mathrm{CO}_{2}$. Time-lapse $z$-series were acquired every 10 minutes for 24 hours with a LD PlanNeofluar 20x/0.4 Korr objective lens.

\section{MGE explant culture and analysis}

Brains from embryonic day 12.5 (E12.5) D/x5/6-ClE;Jnk $1^{\text {tl/ ; Jnk2 }}$ (control) or DIx5/6-

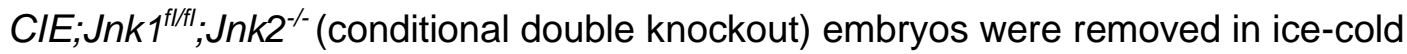
cHBSS, embedded in 3\% low melting point agarose, and sectioned at $300 \mu \mathrm{m}$. Medial ganglionic eminence (MGE) tissue was isolated from appropriate sections and trimmed to generate subventricular zone enriched explants. Each brain was processed individually and yielded approximately two MGE explants plus material for genotyping, which was performed retrospectively. A 1:1 collagen-matrigel mixture was made by preparing a $2 \mathrm{mg} / \mathrm{ml}$ working stock of neutralized collagen (BD Life Sciences, Type 1 Rat Tail High Concentration) in phenol-red free neurobasal medium (Invitrogen) and diluting it 1:1 with phenol-red free matrigel (Corning Life Sciences). Ice-cold collagen-matrigel mixture was added to each well of an eight-well Lab-Tek chambered coverglass, and MGE explants were pipetted into matrix-filled wells. MGE-embedded matrices were allowed to solidify for $45 \mathrm{~min}$ at $37^{\circ} \mathrm{C}$ with $5 \% \mathrm{CO}_{2}$, fed with phenol-red free neurobasal medium supplemented with GlutaMAX (Invitrogen), penicillin-streptomycin (Thermo Scientific), and B-27 (Invitrogen), and cultured for two days. Cultured explants were imaged on a Zeiss LSM 510 confocal microscope at a uniform optical thickness. Area measurements were performed on projected montages in ImageJ. Outgrowth area was determined by subtracting the explant area from the total area occupied by both the explant and migrating cells (Figure 8F), and normalized to explant size. Explants were measured blind to genotype and evaluated statistically by a two-tailed unpaired Student's t-test using Prism 6 (GraphPad) software. 


\section{$\underline{\text { Immunostaining of Slice Cultures }}$}

Slices were fixed overnight in $4 \%$ paraformaldehyde in $1 \times$ phosphate buffered saline (PBS) at $4^{\circ} \mathrm{C}$. Slices were rinsed from fixative with $1 \times \mathrm{PBS}$, and blocked overnight at $4^{\circ} \mathrm{C}$ in permeability solution (Polleux and Ghosh, 2002) with 5\% normal goat serum. Rabbit anti-GFP (Invitrogen; 1:1500) and mouse anti-nestin (BD Transduction Laboratories; 1:1000) primary antibodies were diluted in block, applied to slices, and incubated overnight at $4^{\circ} \mathrm{C}$. Slices were thoroughly rinsed in $1 \times$ PBS, and incubated overnight at $4^{\circ} \mathrm{C}$ with secondary antibody solution containing goat anti-rabbit Alexa488 (1:4000; Invitrogen), goat anti-mouse Alexa546 (1:2000; Invitrogen), and the nucleic acid counterstain Draq5 (1:4000; Axxora) diluted in block. Slices were rinsed in 1x PBS and slide mounted in an aqueous mounting medium containing anti-fade reagent.

\section{Cryosectioning and Staining}

Embryonic day 13.5 (E13.5) heads were removed and fixed with 4\% paraformaldehyde overnight, rinsed in 1x PBS, and progressed through a sucrose series (10, 20,30\%). Cryo-protected heads were embedded with TFM ${ }^{\mathrm{TM}}$ Tissue Freezing Medium (Triangle Biomedical) and flash frozen in liquid-nitrogen-cooled 2-methyl butane. Heads were serially sectioned $(12 \mu \mathrm{m})$ in the coronal plane and slides stored at $-20^{\circ} \mathrm{C}$ prior to use. Slides were rehydrated with $1 \times$ PBS for 20 minutes, blocked (as above) for $2 \mathrm{hrs}$ at room temperature, and incubated in primary antibody solution (block with primary antibodies) overnight at $4^{\circ} \mathrm{C}$. Primary antibodies included rabbit anti-calbindin (Swant; $1: 2000$ ), mouse anti-nestin (BD; 1:1000), and chicken anti-GFP (Abcam; 1:1500). Slides were rinsed with $1 \times$ PBS, incubated with secondary antibody and nucleic acid counterstain solution (as above) for 2 hours at room temperature, rinsed in 1x PBS, and coverslipped with an aqueous mounting medium containing anti-fade reagent. 


\section{Imaging and Analysis}

Immunofluorescently labeled slice cultures and cryosections were imaged on a Zeiss LSM 510 Meta Confocal Microscope with a 10x Fluar or 20x Plan-Apo objective lens. Confocal micrographs were uniformly adjusted for levels, brightness, and contrast in Adobe Photoshop. For quantification of migration, cortical length was measured from the corticostriatal boundary to the cortical arch of each image, and cortices were segmented into 5 equidistant bins (Figures 2.2 and 2.5). Similarly, for quantification of radial distribution (Figure 2.8), a defined region of the lateral cortical wall was cropped from montaged images and equidistantly segmented into six bins from pial to ventricular surfaces. For both studies, the numbers of cells present in each bin were counted and their percentile distributions across all bins were determined for each tissue section. Bin distributions were averaged across sections of the same treatment group or genotype, and statistical significances were determined by two-way ANOVA followed by Fisher's LSD post-hoc analyses (GraphPad Prism 6).

4D live imaging movies were analyzed using Imaris (Bitplane). Movies (12 control and 12 inhibitor) were evaluated in the first 12 hours and last 12 hours of each recording. Ten interneurons were tracked in each time segment for all movies. Interneurons were selected for tracking if they could be followed for at least 4 hours. Tracks were discontinued if a cell remained stationary for 40 contiguous minutes, or if the tracked cell could no longer be unambiguously identified. Average velocity, displacement, and straightness values were obtained for each movie (20 cells). Each treatment group contained 120 cells analyzed from 12 movies $(n=12)$. Two-tailed unpaired Student's $t-$ tests (GraphPad Prism 6) were used to determine statistical differences between groups. 


\section{Results}

Interneurons express Jnk proteins as they first enter the cortical rudiment

We used transgenic mice expressing EGFP under the control of a DIx5/6 Cre-IRESEGFP transgene (hereafter referred to as DIx5/6-CIE) to follow the migration of newly born interneurons into the developing mouse cortex. DIx5/6-CIE+ interneurons first arrive at the entrance to the cortical rudiment on day E12.5 (Figure 2.1A). By E13.5, DIx5/6$\mathrm{CIE}+$ interneurons have crossed the cortico-striatal boundary and have migrated approximately half the length of the lateral cortical wall (Figures 2.1B, C). As they enter the neocortex, DIx5/6-CIE+ interneurons form two medially oriented migratory streams flanking the emerging cortical plate; an upper marginal zone (MZ) stream and lower subventricular zone/intermediate zone (SVZ/IZ) stream (Figure 2.1C). Thus, the first cohort of DIx5/6-CIE+ cortical interneurons forms streams of migratory cells that rapidly enter the cortical rudiment over a single developmental day.

JNK signaling regulates cell survival (Kuan et al., 1999), radial migration (Westerlund et al., 2011), and axon tract formation (Hirai et al., 2006; Yamasaki et al., 2011) in the developing forebrain. However, the extent to which JNK signaling influences the migration of cortical interneurons is unknown. We examined the expression pattern of total Jnk protein between E12.5 and E13.5 to determine whether JNK signaling could regulate the initial migration of cortical interneurons into the cerebral cortex. Jnk protein is widely expressed in the developing forebrain (Figures 2.1A, B), but appears to be enriched in the nascent cortical plate and mantle region of the ventral forebrain, where newly generated post-mitotic neurons reside. Moreover, Jnk proteins are expressed by tangentially migrating $\mathrm{Dl} \times 5 / 6-\mathrm{ClE}+$ cortical interneurons as they enter the cerebral cortex (Figures 2.1D-F). Quantitative reverse transcriptase PCR analysis of FACS sorted 
DIx5/6-CIE+ and DIx5/6-CIE(-) cells from dissociated E14.5 cortices indicated that Jnk1 mRNA is enriched at nearly twofold (average fold change $\pm \mathrm{SEM}=1.99 \pm 0.22$ ) in DIx5/6-CIE + cortical interneurons, while Jnk2 is uniformly expressed in both populations (Figure 2.1H). Thus, DIx5/6-CIE+ cortical interneurons express Jnk proteins as they enter the cortical rudiment and, compared to DIx5/6-CIE(-) cortical cells, selectively express Jnk1 at higher levels.

\section{Interneuron entry into the cortical rudiment requires intact JNK signaling}

To evaluate the initial entry of interneurons into the cerebral cortex under controlled conditions, we prepared live-vibratome slices from E12.5 DIx5/6-CIE+ brains and cultured them for one day in vitro (Figure 2.2A). Initially, very few cortical interneurons were present in the cortical rudiment (Figure 2.2B). Over the next day in vitro (DIV), DIx5/6-CIE+ cortical interneurons rapidly invaded the lateral aspect of the cerebral cortex (Figures 2.2C-D), recapitulating their cortical entry in vivo (Figures 2.1A-C).

To determine whether interneuron entry into the cerebral cortex depends on intact JNK signaling, we cultured E12.5 DIx5/6-CIE+ slices for one DIV in the presence of different concentrations of SP600125, a selective, reversible, pan inhibitor of JNK signaling (Bennett et al., 2001). At $1 \mu \mathrm{M}$, SP600125 had little effect on interneuron entry into the cortex (Figure 2.2E). As the concentration of SP600125 was increased from $5 \mu \mathrm{M}$ to 40 $\mu \mathrm{M}$, however, a dose dependent inhibition of interneuron migration into the cerebral cortex was observed (Figures 2.2F-H). To quantify interneuron entry into the cortex, we determined the frequency in which DIx5/6-CIE+ interneurons were found in five equidistant cortical bins (Figure 2.2I). When compared to control sections, interneurons from SP600125-treated sections showed a dose-dependent accumulation in the most lateral cortical bin, and dose-dependent reductions in medial bin locations (Figure 2.2J). 
Thus, pharmacological blockade of JNK signaling prevents the initial entry of DIx5/6$\mathrm{ClE}+$ cortical interneurons into the cerebral cortex in a dose-dependent fashion.

Blockade of interneuron entry into the cerebral cortex by JNK inhibition is specific and reversible

To determine whether disruption of cortical interneuron migration was specific to JNK inhibition, we evaluated whether pharmacological blockade of the MAPK signal transduction cascade had a similar effect on the initial entry of cortical interneurons into the cortical rudiment. Unlike JNK inhibition, U0126, a potent and selective inhibitor of Mek1/2 (Favata et al., 1998), failed to prevent the entry of Dlx5/6-ClE + interneurons into the cerebral cortex (Figure 2.3C). Indeed, no differences were found between the advancement of DIx5/6-ClE+ interneurons in slices treated with $10 \mu \mathrm{M} U 0126$ and control slices (Figure 2.3D), confirming previous assessments of U0126 on cortical interneuron migration (Polleux et al., 2002).

To further validate the specificity of JNK inhibition on cortical interneuron migration, we cultured E12.5 DIx5/6-CIE+ slices for 24 hours in the presence of AS601245, an independent pan-inhibitor of JNK signaling (Carboni et al., 2008). Similar to SP600125 treatment, application of AS601245 significantly impaired entry of Dlx5/6-CIE+ interneurons into the cortical rudiment (Figures 2.3B, D). Indeed, a $5 \mu \mathrm{M}$ concentration of AS601245 impaired interneuron entry to an extent approximating a $20 \mu \mathrm{M}$ concentration of SP600125 (Figures 2.2G, 2.3B). Thus, application of two independent inhibitors of JNK signaling prevented interneuronal migration into the nascent cortical rudiment, while pharmacological inhibition of MAPK signaling had no effect, suggesting JNK signaling, but not MAPK signaling, is required for the initial migration of cortical interneurons into the cortical rudiment. 
We next asked whether blockade of interneuron migration into the cerebral cortex by SP600125 treatment was reversible. E12.5 DIx5/6-CIE+ slices were exposed to $20 \mu \mathrm{M}$ SP600125 for 12 hours, rinsed, and grown for an additional 12 hours in either control medium ("washout" condition; Figure 2.3H) or medium containing freshly prepared 20 4M SP600125 (“SP600125” condition; Figure 2.3G). When compared to control slices (Figure 2.3F), slices in both washout and SP600125 conditions displayed impaired migration (Figures 2.3G, H). Dlx5/6-CIE+ interneurons showed robust recovery in washout conditions, however, and migrated much further into the cortical rudiment than DIx5/6-CIE+ interneurons in slices continuously exposed to SP600125 (Figures 2.3E-H). Thus, SP600125 treatment impairs interneuron entry into the cortex, but this effect is largely reversible upon removal of JNK inhibition.

\section{JNK inhibition impairs directed migration of cortical interneurons into the cortical rudiment}

To further examine consequences of JNK inhibition on the migration of cortical interneurons, time-lapse recordings were made from E12.5 DIx5/6-CIE+ slices exposed to control medium or medium containing $20 \mu \mathrm{M}$ SP600125. Live images of the cortical entry zone and lateral aspect of the cortical rudiment were acquired every 10 minutes for 24 hours in control and JNK-inhibited conditions, and migratory properties of Dlx5/6$\mathrm{CIE}+$ cortical interneurons were examined as they entered and navigated the cerebral cortex.

In control slices, DIx5/6-CIE+ interneurons robustly entered the cortical rudiment and traveled tangentially to fill the lateral cortical wall (Figures 2.4A-D; Movie 2.1, video clip 1). Many DIx5/6-CIE+ interneurons traversed the cortical entry zone by 12 hours of recording (Figure 2.4C), and by 24 hours, Dlx5/6-CIE+ interneurons had formed $M Z$ and 
SVZ/IZ streams on their medial progression through the cortex (Figure 2.4D), often migrating beyond the field of view.

In stark contrast, migration of $\mathrm{Dl} \times 5 / 6-\mathrm{CIE}+$ interneurons through the cortical entry zone was severely compromised when slices were treated with $20 \mu \mathrm{M}$ SP600125 (Figures 2.4E-H; Movie 2.2, video clip 1). Dlx5/6-ClE+ interneurons remained motile, yet failed to advance in medially oriented trajectories. After 12 hours, far fewer Dlx5/6-ClE+ interneurons had entered the cortex (Figure 2.4G) compared to control conditions (Figure 2.4C). By 24 hours, progression of DIx5/6-ClE+ interneurons into the cortex had improved (Figure $2.4 \mathrm{H}$ ), but failed to match the extent of interneuron migration seen in controls (Figure 2.4D), as previously quantified (Figure 2.2J). Dlx5/6-CIE+ cells often stacked up in the MZ stream (Figure 2.4H), and remained dispersed in the SVZ/IZ region without forming an organized migratory stream.

To quantify changes in migratory behavior, individual DIx5/6-CIE+ interneurons were analyzed in control and SP600125-treated slices. Representative cell tracks of cortical interneurons in control (Figures 2.4I-J; Movie 2.1, video clips 2-3) and JNK-inhibited (Figures 2.4K-L) conditions are shown for movies depicted in Figure 2.4. Navigational errors were apparent during the first 12-hours of imaging in SP600125-treated slices, when Dlx5/6-ClE+ interneurons stall their advancement at the entrance to the cortex, and take short, aberrant paths toward the pial or ventricular surfaces (Figure 2.4K; Movie 2.2, video clip 2). Lengths of individual cell tracks (track length) increased during the last 12-hours of imaging in SP600125-treated slices, but DIx5/6-CIE+ interneurons typically remained misguided (Figure 2.4L; Movie 2.2, video clip 3), and the entire migratory front of cortical interneurons remained less advanced compared to controls. 
For statistical comparisons, 240 migratory interneurons from 12 control and 12 SP600125-treated slices were tracked and their trajectories were analyzed. Twenty DIx5/6-CIE+ interneurons were tracked from each slice: 10 during the first 12 hours, and 10 during the last 12 hours of imaging. Speed of interneuron advancement significantly declined after SP600125 treatment, with greatest deficits occurring during the first 12hour period (Figure 2.4M). Maximum, mean, minimum, and standard deviation velocities of DIx5/6-CIE+ interneurons diminished significantly in SP600125-treated slices (values $=\operatorname{avg} \pm \mathrm{SEM}$; Control: $\max =139.6 \pm 4.4$, mean $=59.6 \pm 2.0, \min =7.6 \pm 0.5, \mathrm{SD}=35.1$ $\pm 1.0 \mu \mathrm{m} / \mathrm{hr} ; \underline{\mathrm{SP} 600125}: \max =90.3 \pm 3.3$, mean $=38.5 \pm 1.8, \min =4.9 \pm 0.4, \mathrm{SD}=21.8$ $\pm 0.9 \mu \mathrm{m} / \mathrm{hr}$ ). Accordingly, average track lengths from the beginning to ending point of their trajectories (displacement) as well as average cumulative distances traveled (total) were significantly impaired during the first 12-hour period (Control: displacement $=204.6$ \pm 8.1, total $=297.2 \pm 12 \mu \mathrm{m} ; \underline{\text { SP600125: }}$ displacement $=98.9 \pm 8.3$, total $=208.4 \pm 10.7$ $\mu \mathrm{m})($ Figure 2.4N). Migratory velocities of interneurons in SP600125-treated slices increased during the last 12-hours of imaging (Figure 2.4P), but remained significantly lower than controls ( Control: $\max =129.6 \pm 2.9$, mean $=54.3 \pm 1.7, \min =7.0 \pm 0.6, S D$ $=32.8 \pm 0.8 \mu \mathrm{m} / \mathrm{hr} ; \underline{\mathrm{SP} 600125}: \max =107.8 \pm 2.7$, mean $=44.5 \pm 1.6, \min =4.9 \pm 0.5$, $\mathrm{SD}=26.8 \pm 0.8 \mu \mathrm{m} / \mathrm{hr}$ ). As velocity increased during the last 12 hours of imaging, so did distances traveled by individual cortical interneurons. Although both displacement and total track lengths increased, displacement length remained reduced to statistically significant levels in the last 12-hours of imaging (Control: displacement $=197.6 \pm 6.4$, total $=270.5 \pm 8.4 \mu \mathrm{m} ; \underline{\mathrm{SP} 600125}$ : displacement $=170.6 \pm 11.3$, total $=261.7 \pm 12.6 \mu \mathrm{m})$ (Figure 2.4Q). Directionality of migration as reflected in overall track straightness values, however, was significantly altered in interneurons from SP600125-treated slices during both first (Control: $r=0.70 \pm 0.01$; SP600125: $r=0.47 \pm 0.02$ ) and last 12-hour intervals

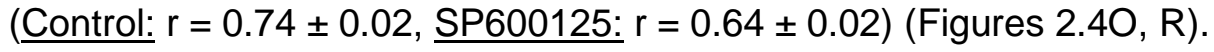


Improvements observed in migratory speed and track length during the last 12 hours of imaging in SP600125-treated slices likely reflect declining efficacy of the pharmacological inhibitor over the 24-hour imaging period. When interneurons were allowed to grow into the cerebral cortex for 12 hours in control conditions prior to treatment with SP600125, deficits in interneuron migration were comparable to those observed in acutely treated slices. For example, mean interneuron velocity was reduced to $37.2 \pm 1.2 \mu \mathrm{m} / \mathrm{hr}$, which was slightly less than the mean velocity observed during the first 12 hours of SP600125 treatment. Furthermore, to evaluate whether the JNK requirement persisted beyond cortical entry at $\mathrm{E} 12.5$, we analyzed migratory properties of control and JNK-inhibited cortical interneurons at the leading front of migration in E14.5 cortices. Similar to SP600125 treatment at E12.5, pharmacological inhibition of JNK at E14.5 led to statistically significant reductions in total track length (Control: 267.3 \pm 17.4 ; SP600125: $188.1 \pm 8.5 \mu \mathrm{m} ; \mathrm{p}=0.01^{*}$ ), mean velocity (Control: $51.7 \pm 0.8$; SP600125: $32.1 \pm 1.6 \mu \mathrm{m} / \mathrm{hr} ; \mathrm{p}=0.0004^{* \star *}$ ), and directionality (Control: $0.75 \pm 0.05$; SP600125: $\left.0.56 \pm 0.05 ; p=0.04^{*}\right)$. Thus, inhibition of JNK signaling significantly slows the advancement and alters the trajectories of migratory cortical interneurons in the cortical entry zone at E12.5 and at the leading front of migration in E14.5 cortices.

Jnk1 regulates cortical interneuron migration at the cortical entry zone in vivo

In order to determine whether loss of Jnk genes compromised migration of cortical interneurons in vivo, we evaluated the distribution of calbindin-expressing cortical interneurons in an allelic series of Jnk1 and Jnk2 single and combinatorial mutant embryos at E13.5 (Figures 2.5 and 2.6), after the initial cohort of interneurons have entered the cerebral cortex. At this age, calbindin expression labels most tangentially migrating interneurons in the cortical rudiment (Anderson et al., 1997). Five embryos of each genotype were coronally sectioned, and the lateral cortical wall was bilaterally 
sampled at four rostro-caudal locations spanning sections containing the rostral MGE to mid caudal ganglionic eminence (CGE; Figure 2.5A). The cortical rudiment was subdivided into 5 equidistant bins spanning the cortico-striatal boundary laterally (Bin1) to cortical arch medially (Bin5; Figure 2.5A), and the percentage of calbindin-positive cortical interneurons found in each cortical bin was determined for each section and averaged across all embryos of the same genotype. Since placement of equidistant bins was done with respect to the length of each cortical hemisphere, our sampling strategy accounts for variation in cortical length that might occur between sections.

In Jnk ${ }^{+/-}$embryos, calbindin-positive interneurons displayed normal tangential migration into the cortical rudiment at both rostral and caudal probe locations (Figure 2.5B, F). However, calbindin-positive interneurons in both MZ and SVZ/IZ streams of Jnk $1^{-\%}$ embryos (Figures 2.5C, G) were less advanced than calbindin-positive interneurons in

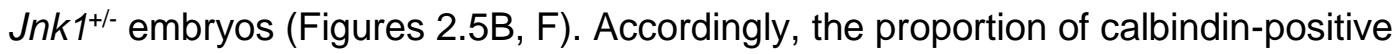
interneurons increased laterally (Bin1) and declined medially (Bin3-5; Figures 2.5E, I). In this and subsequently presented data, statistically significant increases in interneuron abundance are seen in Bin1 and statistically significant decreases in interneuron abundance are seen in Bin3 when migratory deficits are present. Bin2 likely remains unchanged due to transitional normalization occurring between Bins 1 and 3 , and while reductions of interneuron abundance occur in Bins 4-5, they often do not reach statistical significance due to the relatively small percentages of cells found in those bins at E13.5. Although reductions of interneuron migration were apparent at both rostral and caudal probe locations in $\mathrm{Jnk}^{-\%}$ embryos, reductions in medial progression only reached statistical significance at caudal probe locations (Figure 2.5E, 2.5I).

To determine whether reduction of Jnk2 in combination with loss of Jnk1 exacerbated migratory phenotypes of cortical interneurons, we analyzed the distribution of calbindin- 
positive cortical interneurons in embryos lacking Jnk1 and a single genomic copy of Jnk2 $\left(J n k 1^{-/} ; J n k 2^{+-}\right)$, since Jnk1/2 double nulls die prior to interneuron migration into the cerebral cortex (Kuan et al., 1999). In Jnk $1^{-/-} ;$Jnk2+/- embryos, advancement of calbindinpositive interneurons in both MZ and SVZ/IZ streams was significantly diminished relative to $J n k 1^{+/}$controls at both rostral and caudal probe locations (Figures 2.5D, H). Statistically significant interactions were found between genotype and bin position at rostral and caudal probe locations (Figures 2.5E, I), suggesting loss of Jnk1 alone and loss of Jnk1 combined with reduction of Jnk2 inhibits tangential progression of cortical interneurons in vivo. At caudal locations, calbindin-positive interneurons in Jnk $1^{-/} ; \mathrm{Jnk} 2^{+/-}$ and $J n k 1^{-/-}$embryos were comparably regressed (Figures $2.5 \mathrm{G}-\mathrm{I}$ ), but at rostral locations, calbindin-positive interneurons in $\mathrm{Jnk}^{-1-} ; \mathrm{Jnk} 2^{+--}$embryos were significantly less advanced than those in $J n k 1^{-/}$embryos (Figures 2.5C-E). To ensure that potential differences in brain size did not influence interpretation of our results, we measured absolute cortical lengths from every section we analyzed and found no statistically significant differences in cortical length between genotypes by one-way ANOVA (Jnk $1^{+/-}$ $\left.=937.8 \pm 17.4, \mathrm{Jnk} 1^{-/}=1005.0 \pm 21.0, \mathrm{Jnk} 1^{-/} ; \mathrm{Jnk} 2^{+/-}=935.4 \pm 27.6 \mu \mathrm{m}\right)$. Thus, at rostral positions, reducing Jnk2 in combination with the loss of Jnk1 has a greater impact on cortical interneuron migration than loss of Jnk1 alone.

To determine whether Jnk2 plays a comparable role to Jnk1 in the initial migration of cortical interneurons in vivo, we generated embryos with the reciprocal Jnk2 $2^{+-}$, Jnk2

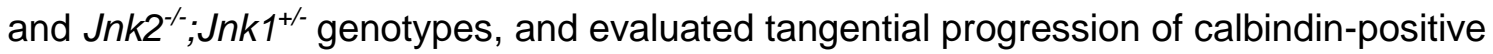
cortical interneurons at E13.5. Normal migration of cortical interneurons was observed in

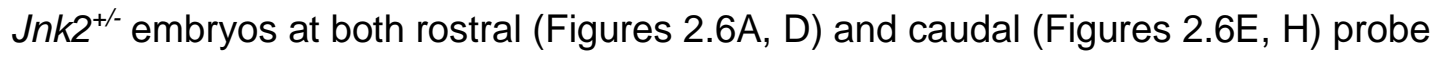
locations, and their cortical bin distribution closely matched $\mathrm{Jnk} \mathrm{1}^{+-}$embryos (Figures 2.5E, I). Unlike in $\mathrm{Jnk} 1^{-/-}$embryos, however, tangential progression of calbindin-positive 
interneurons was completely unperturbed in Jnk2-/- embryos at rostral (Figures 2.6B, D) and caudal probe locations (Figures 2.6F, H). Moreover, removing a genomic copy of Jnk1 in the context of Jnk2 deletion did not alter the advancement of calbindin-positive cortical interneurons (Figures 2.6C, D, G, H). Neither MZ nor SVZ streams were

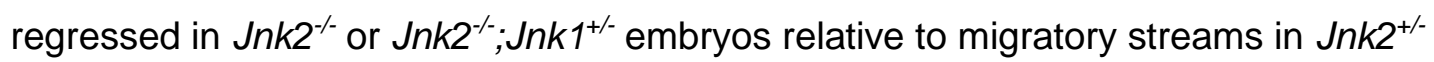
controls (Figures 2.6A-G), and the distribution of calbindin-positive cortical interneurons in cortical bins were nearly identical across all three genotypes at rostral and caudal locations (Figures 2.6D, H). As before, cortical rudiment lengths were consistent

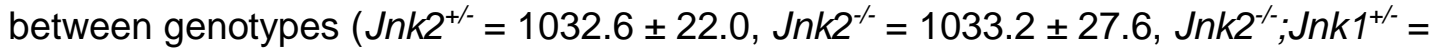
$1014.5 \pm 9.3 \mu \mathrm{m})$. Apparently, retaining a single genomic copy of $J n k 1$ is sufficient to leave cortical interneuron migration intact and unperturbed in the Jnk2-/-background at E13.5.

\section{Cortical interneurons have a cell-intrinsic requirement for Jnk1 in vivo}

Genetic ablation of Jnk1 disrupts the initial migration of interneurons into the cortical rudiment, but since Jnk1 is expressed in both migratory cortical interneurons and noninterneuronal cells of the developing cortex (Figure 2.1), the requirement for JNK signaling in regulating cortical interneuron migration may not be cell autonomous. Indeed, radial migration of cortical projection neurons is accelerated in $\mathrm{Jnk}^{1-}$ embryos (Westerlund et al., 2011), which could lead to non-autonomous disruptions in the tangential migration of cortical interneurons. To determine whether the initial cohort of cortical interneurons has a cell-intrinsic requirement for JNK signaling to migrate into the cortical rudiment, we used mice expressing the DIx5/6-CIE transgene to conditionally ablate Jnk1 within cortical interneurons in vivo. Since migration of cortical interneurons was completely unperturbed after constitutive loss of $J n k 2^{-\sim}$ (Figure 2.6), but reduction of Jnk2 in Jnk1 nulls exacerbated interneuron migratory phenotypes (Figure 2.5), we 
conditionally ablated Jnk1 in interneurons of Jnk2 ${ }^{-/}$embryos. This allowed evaluation of interneurons that were completely deficient in both Jnk1 and Jnk2, which would otherwise be impossible due to the mid-gestation embryonic lethality of constitutive Jnk1/2 double mutation.

We predicted DIX5/6-CIE;Jnk $1^{f / /} ;$ Jnk2 $2^{-/}$embryos would not have deficiencies in interneuron migration, since interneuron migration was unperturbed in Jnk2-- ;Jnk $1^{+-}$ embryos. Indeed, normal tangential migration of cortical interneurons was observed at E13.5 in DIx5/6-CIE;Jnk1 ${ }^{f / /+} ;$ Jnk2 $2^{-/}$embryos (Figures 2.7A, E). We analyzed DIx5/6-CIE+ cortical interneurons, as well as the calbindin-expressing cohort of cortical interneurons, which constitute most of the DIx5/6-CIE+ cells in the cortical rudiment at E13.5. Both populations of labeled cells appeared normally distributed within the cortex of $D / x 5 / 6$ CIE;Jnk $1^{f l /} ;$ Jnk2 ${ }^{-/-}$embryos at rostral (Figures 2.7C, D) and caudal probe locations (Figures 2.7G, H). In DIx5/6-CIE;Jnk $1^{f / / f l} ;$;nk2 $2^{-/-}$embryos, however, both DIx5/6-CIE+ and calbindin-positive cortical interneurons were significantly regressed in their cortical advancement at rostral (Figures 2.7B-D) and caudal (Figures 2.7F-H) probe locations compared to interneurons in control brains. The distribution of interneurons within DIX5/6-CIE;Jnk1 $1^{f l f l i} ; J n k 2^{-/-}$cortices were significantly altered from control DIx5/6CIE;Jnk $1^{f l /+} ;$ Jnk2 $2^{-/-}$cortices at rostral (Figures 2.7C, D) and caudal probe locations (Figures 2.7G, H). Similar to $J n k 1^{--}$and $J n k 1^{-/} ; J n k 2^{+/}$embryos, cortical interneurons accumulated laterally and diminished medially within DIX5/6-CIE;Jnk $1^{f / f l} ;$ Jnk2 ${ }^{-/}$cortices, with the largest statistical differences between genotypes occurring in Bin1 and Bin3 (Figures 2.7C, D, G, H). Distributions of cortical interneurons within D/x5/6CIE;Jnk $1^{f / / f l} ; J n k 2^{-/-}$embryos were comparable to $J n k 1^{-/-} ; J n k 2^{+--}$embryos since both rostral and caudal portions of their trajectories were significantly regressed relative to their respective controls (Figures $2.5 \mathrm{E}, \mathrm{I}$ ). This finding is consistent with Jnk2 loss of function 
enhancing a $J n k 1^{-/-}$migratory phenotype at rostral probe locations. Cortical lengths were not significantly different between control and conditional Jnk1/2 double knockout embryos (DIx5/6-CIE;Jnk $1^{f / /} ;$ Jnk2 ${ }^{-/}=949.1 \pm 50.1$, DIx5/6-CIE;Jnk $1^{f / / f l} ; J n k 2^{-/}=922.7 \pm$ $56.0 \mu \mathrm{m})$. Collectively, these data suggest the initial cohort of cortical interneurons has a cell-intrinsic requirement for JNK signaling —largely mediated through the activity of Jnk1, but partially compensated for by Jnk2 when Jnk1 is lost-in migrating through the cortical entry zone in vivo.

Although our results indicate cortical interneurons have a cell-intrinsic requirement for JNK signaling to appropriately enter the cerebral cortex, there are two possible explanations for this requirement: 1) JNK signaling could intrinsically enhance the migratory capacity of cortical interneurons, or 2) JNK signaling could be required by cortical interneurons to sense or respond to environmental cues located in the cortical rudiment. To distinguish between these possibilities, we cultured explants of MGE tissue from E12.5 control or conditional double knockout embryos in a reduced, serum-free in vitro environment devoid of cortical cues (Figure 2.8A). MGE explants isolated from

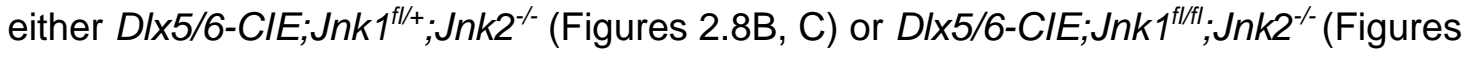
2.8D, E) forebrain slices produced DIx5/6-CIE+ migratory interneurons that dispersed in a radial pattern from the explant margins. When migratory outgrowth was measured blind to genotype (Figure 2.8F), no statistical differences in interneuron migration were

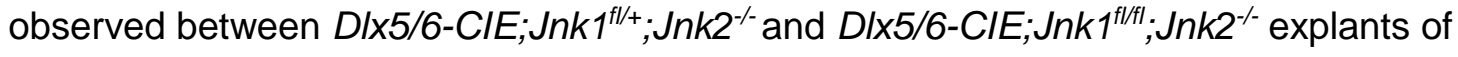
MGE tissue (Figure 2.8G). These data strongly suggest that JNK signaling does not act in cortical interneurons to autonomously promote or enhance their motility. 


\section{Integrity of cortical migratory streams depends on JNK signaling in vivo}

Since we observed statistically significant deficits in the entry of cortical interneurons into the cortical rudiment after conditionally ablating Jnk1 from interneurons of Jnk2-null mutants in vivo (Figure 2.7), but failed to see migratory anomalies in the absence of cortical cues in vitro (Figure 2.8), we hypothesized that cortical interneurons have a cell intrinsic requirement for JNK signaling to navigate the early cortical rudiment in vivo. To determine whether JNK-deficient cortical interneurons are misrouted when first entering the cerebral cortex, we quantified the radial distribution of cortical interneurons within the

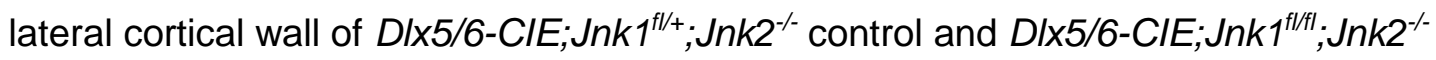
mutant embryos at E13.5 (Figures 2.9A-C). Six equidistant bins were made along the radial axis of the lateral cortex (adjacent to the corticostriatal boundary), and the percentage of DIx5/6-CIE+ cortical interneurons appearing in Bins 1 (MZ) through 6 (Ventricular Zone; VZ) were determined for each genotype. In D/x5/6-CIE;Jnk $1^{t / /+} ; \mathrm{Jnk} 2^{-/-}$ embryos, DIx5/6-CIE+ interneurons were predominantly distributed in the MZ (Bin 1) and

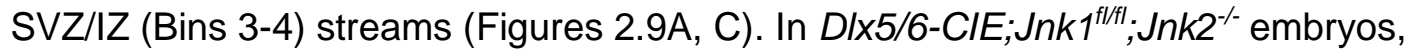
however, the proportions of Dlx5/6-CIE+ interneurons located in the MZ stream (Bin 1) and SVZ portion of the SVZ/IZ stream (Bin 4) were diminished compared to control cortices (Figures 2.9B, C). In addition, the percentage of DIx5/6-ClE+ interneurons in the IZ (Bins 2-3) was elevated in conditional double mutants compared to controls (Figures $2.9 \mathrm{~B}, \mathrm{C}$ ). When compared statistically, distributions of Dlx5/6-CIE+ migratory interneurons within Bins 1-6 were significantly altered between D/x5/6-CIE;Jnk $1^{f / /+} ;$ Jnk $2^{-1-}$ and DIX5/6-CIE;Jnk $1^{f / f / l} ; J n k 2^{-/}$genotypes with the largest shifts occurring in Bins 3 and 4 (Figure 2.9C), suggesting JNK-deficient interneurons were dispersing from the SVZ.

Moreover, repositioning of migratory interneurons within the radial axis of the $D / x 5 / 6$ CIE;Jnk1fl/fl;Jnk2-/- mutant cortex was accompanied by diminished integrity to both MZ 
and SVZ/IZ migratory streams. The MZ and SVZ/IZ streams of DIx5/6-CIE;Jnk $1^{t / /+} ; \mathrm{Jnk} 2^{-1-}$ control embryos (Figures 2.9D, E) were consistently more cohesive than MZ and SVZ/IZ streams of DIx5/6-CIE;Jnk $7^{t / f / l} ;$ Jnk2 ${ }^{-/}$mutant embryos (Figures $2.9 \mathrm{H}, \mathrm{I}$ ). Dlx5/6-CIE+ and calbindin-positive cortical interneurons were more loosely organized in $D / x 5 / 6$ -

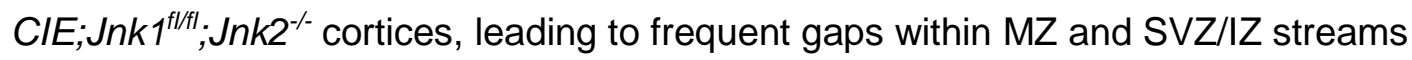
(Figures $2.9 \mathrm{H}, \mathrm{I}$ ). Leading processes of cortical interneurons within the SVZ/IZ stream of DIx5/6-CIE;Jnk $1^{\text {t/l+; Jnk2 }}{ }^{-/}$cortices were typically straight and medially oriented (Figure 2.9E), while disordered and non-tangentially oriented in the SVZ/IZ stream of D/x5/6CIE; Jnk $1^{t / f / t} ; J n k 2^{-/-}$mutant cortices (Figure 2.9I). In addition, interneuronal processes were often non-contiguous with their cell bodies (Figure 2.9l), suggesting migratory orientations of $D / \times 5 / 6-C I E ; J n k 1^{f / / f l} ; J n k 2^{-/-}$interneurons were frequently orthogonal to the coronal plane of section. Finally, processes of cortical interneurons in $D / \times 5 / 6-$

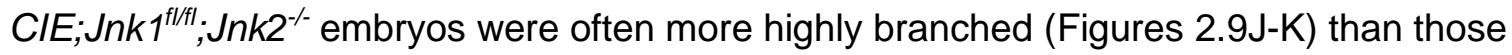
of control interneurons (Figures 2.9F-G). Thus, conditional ablation of Jnk1 from interneurons of $\mathrm{Jnk}^{-/}$embryos disrupts the radial distribution of cortical interneurons at the entrance to the cortical rudiment, diminishes the integrity of MZ and SVZ/IZ migratory streams, and leads to morphological alterations in migratory cortical interneurons. Collectively, these data suggest cortical interneurons have an intrinsic requirement for JNK signaling in navigating the cortical entry zone in vivo. 


\section{Discussion}

In the current study, we identified novel and essential roles for JNK signaling in cortical interneuron migration. We established that cortical interneurons express Jnks as they enter the cerebral cortex and, compared to non-interneuronal cortical cells, have enriched expression of Jnk1. Using ex vivo slice cultures, we demonstrated pharmacological blockade of JNK results in dose-dependent disruption of interneuron migration into the cerebral cortex, and that JNK-inhibited cortical interneurons slow their advancement and take aberrant trajectories as they navigate the cortical entry zone at E12.5. JNK-inhibition at E14.5 similarly perturbed migratory properties of cortical interneurons, indicating their requirement for JNK persists at later embryonic stages. In vivo analyses of single and multi-allelic mutations of two Jnk genes revealed that loss of Jnk1 impairs cortical interneuron migration, while loss of Jnk2 does not, unless Jnk2 is reduced in Jnk1 nulls. Finally, we showed conditional deletion of Jnk1 in interneurons of Jnk2 nulls recapitulates migratory deficits observed in constitutive Jnk ${ }^{-1} ; J_{n k 2}{ }^{+1-}$

embryos, and in addition, disrupts migratory streams and cortical interneuron morphology. Despite this clear intrinsic requirement for JNK activity in vivo, JNKdeficient interneurons migrate normally in vitro, suggesting JNK regulates cortical interneuron migration in response to cortical guidance cues. Together, our results suggest that cortical interneurons have an intrinsic requirement for Jnk1 to enter and navigate the developing cerebral cortex, making the JNK signaling pathway a key intracellular mediator of cortical interneuron migration in vivo.

\section{JNKs and neuronal migration}

JNK signaling orchestrates diverse cellular processes in the developing nervous system including cell survival (Kuan et al., 1999; Sabapathy et al., 1999), axon elongation and 
stability (Hirai et al., 2006; Wang et al., 2007; Yamasaki et al., 2011), axon guidance (Qu et al., 2013), and radial migration (Hirai et al., 2006; Wang et al., 2007; Westerlund et al., 2011; Yamasaki et al., 2011). The extent to which individual Jnk genes have distinct functions remains to be elucidated (Haeusgen et al., 2009; Yamasaki et al., 2012). Here, we report Jnk1 plays a greater role than Jnk2 in cortical interneuron migration. This may either reflect functional differences between Jnk1 and Jnk2, or unequal expression of Jnk1 and Jnk2 in migratory cortical interneurons. The contribution of Jnk3 to cortical interneuron migration is currently unknown.

Radial migration in the developing cortex relies on JNK signaling, yet it is unclear whether Jnk1, Jnk2, and Jnk3 play equivalent roles. Deletion of upstream JNK activators including Map3k12 (Hirai et al., 2006), Map2k4 (Wang et al., 2007), or Map2k7 (Yamasaki et al., 2011), inhibits radial migration. Surprisingly, deletion of Jnk1 has the opposite effect: radial migration into the cortical plate is accelerated in Jnk1 nulls (Westerlund et al., 2011). These conflicting results could be explained if Jnk2 and/or Jnk3 play opposite roles to Jnk1 in radial migration, since deletion of upstream JNK activators would diminish activity of all available Jnk isoforms, whereas single deletion of Jnk1 would not. We find pharmacological inhibition of all JNK activity, as well as genetic deletion of Jnk1 or Jnk1 and Jnk2 together, inhibits tangential progression of cortical interneurons. These results indicate that at least Jnk1 and Jnk2 act in parallel to promote cortical interneuron migration in vivo. Thus, both radial and tangential migration requires Jnk1-mediated signaling, but Jnk1 function exerts opposite effects on radially versus tangentially migrating neuroblasts. Differential expression of downstream JNK targets may underlie the molecular bases of these distinctions. For example, the microtubule regulatory protein SCG10/stathmin-2 is a major downstream effector of Jnk1 in radially 
migrating neuroblasts (Westerlund et al., 2011); cortical interneurons may rely on other JNK substrates, however.

\section{JNK signaling at the "cortical entry zone"}

Our data suggest the first cohort of cortical interneurons require JNK activity to enter and navigate the lateral cortical rudiment. We call this segment of their migratory pathway the "cortical entry zone," which bridges the subcortical telencephalon and neocortex. Extracellular guidance cues located at the cortical entry zone likely direct and concentrate cortical interneurons into newly emerging migratory streams. Indeed, Cxcl12 is highly expressed within the SVZ/IZ at the cortical entry zone (Stumm et al., 2003, 2007; Tiveron et al., 2006; López-Bendito et al., 2008), and is required for interneuron migration into the cortex via the SVZ/IZ stream (Tiveron et al., 2006; López-Bendito et al., 2008). Thus, cortical interneurons respond to extracellular guidance cues located at the cortical entry zone to correctly navigate the nascent cerebral cortex, and our data suggest JNK signaling critically regulates the spatial and temporal precision for which this occurs.

We provide substantial genetic evidence indicating that JNK signaling regulates interneuron migration at the cortical entry zone in vivo (Figure 2.10). Constitutive ablation of Jnk1 diminishes cortical interneuron migration at E13.5, with significant reductions in migration occurring at caudal cortical locations. Loss of Jnk2 alone or loss of Jnk2 with heterozygous reduction of Jnk1 has no discernable effect, however, suggesting a single genomic copy of Jnk1 is sufficient to maintain normal migratory properties of cortical interneurons-even in the absence of Jnk2. In contrast, heterozygous reduction of Jnk2 in Jnk1 nulls impairs cortical interneuron migration more than loss of Jnk1 alone, since tangential migration into both rostral and caudal portions 
of their cortical trajectory are compromised. Thus, compared to Jnk1, Jnk2 plays a relatively minor role in cortical interneuron migration, and its influence is only apparent rostrally when Jnk1 function is lost. Conditional deletion of Jnk1 in interneurons of Jnk2 nulls, which completely eliminates Jnk1 and Jnk2 function in cortical interneurons, significantly impairs cortical interneuron migration throughout the rostral-caudal axis of the developing cortex. Not only is cortical entry compromised when Jnk1 and Jnk2 function is eliminated from cortical interneurons, but MZ and SVZ/IZ streams are less cohesive, radial positioning of migratory interneurons is altered, and migratory cortical interneurons are more highly branched. In a reduced in vitro environment, however, JNK-deficient interneurons migrate normally, suggesting cortical interneurons require JNK to sense or respond to cortical guidance cues rather than promote motility. Taken together, our in vivo genetic, ex vivo pharmacologic, and in vitro migration data suggest cortical interneurons have a cell intrinsic requirement for JNK signaling to guide their migration into and within the developing cerebral cortex.

Two cell biological processes control tangential migration of cortical interneurons: 1) dynamic remodeling of the leading process through branching and elongation, and 2) translocation of the nucleus into the leading process, or nucleokinesis. Both events require precise molecular control of actin and microtubule cytoskeleton (Bellion et al., 2005; Godin et al., 2012), and must be coordinated in a stepwise fashion to orient migration towards chemoattractant guidance cues (Martini et al., 2009). Although cellular and molecular mechanisms underlying the requirement for JNK in migrating cortical interneurons are currently unknown, we hypothesize JNK signaling acts as a molecular bridge between cortically encountered guidance cues and cytoskeletal machinery driving migration. One potential mediator is doublecortin, a microtubule-associated protein regulated by JNK phosphorylation (Gdalyahu et al., 2004; Jin et al., 2010), and required 
for proper branching, nucleokinesis, and directed migration of cortical interneurons (Kappeler et al., 2006; Friocourt et al., 2007). The degree to which extracellular guidance cues activate JNK signaling to modulate doublecortin, or other regulators of cytoskeletal dynamics in migrating cortical interneurons, such as Lis1 (Gopal et al., 2010), p27 Kip1 (Martini et al., 2009), or Rac-GTPases (Tahirovic et al., 2010; Tivodar et al., 2015), remains to be determined.

\section{Relevance to neuropsychiatric disorders}

Cortical interneurons are cellular targets for the pathogenesis of several developmental disorders of cortical connectivity, including schizophrenia and autism (Marín, 2012). Recent work in a mouse model of 22q11.2 deletion syndrome (22q11.2DS), which confers significant genetic risk for schizophrenia and autism in humans (Murphy et al., 1999; Fine et al., 2005; Métin et al., 2006; Niklasson et al., 2009), has revealed that embryonic migratory deficits in cortical interneurons lead to laminar repositioning of parvalbumin-positive interneurons in adult cortices (Meechan et al., 2009, 2012). Similar to JNK-deficient interneurons, cortical interneurons in 22q11.2DS brains display delayed cortical entry at E13.5 and irregularities in their migratory streams (Meechan et al., 2009, 2012). Interneuron migratory deficiencies in 22q11.2DS mice are cell autonomous and largely result from reductions in the chemokine receptor Cxcr4 (Meechan et al., 2012). Independent analysis of a similar mouse model confirmed that functional defects in Cxcl12/Cxcr4 signaling underlie interneuron deficiencies in 22q11.2DS mice (Toritsuka et al., 2013), and implicated Dgcr8 as the mediator. It is currently unknown whether genes within the 22q11.2 locus, including Dgcr8, interact with the JNK pathway, but similarities in interneuron migratory deficits are highly suggestive of a connection. One plausible explanation is that $\mathrm{Cxcl12-Cxcr4}$ signaling activates JNK to guide interneuron 
migration and promote migratory stream integrity at the cortical entry zone, but this hypothesis remains to be tested.

Uncovering cellular and molecular mechanisms underlying the pathogenesis of cortical connectivity disorders is essential for developing effective strategies for their treatment. Our current work identifies the JNK signaling pathway, and Jnk1 in particular, as a major regulator of interneuron migration in vivo-making the JNK pathway a novel candidate to consider in the etiology of cortical circuit disorders and a target for potential therapeutic interventions. 
Figures
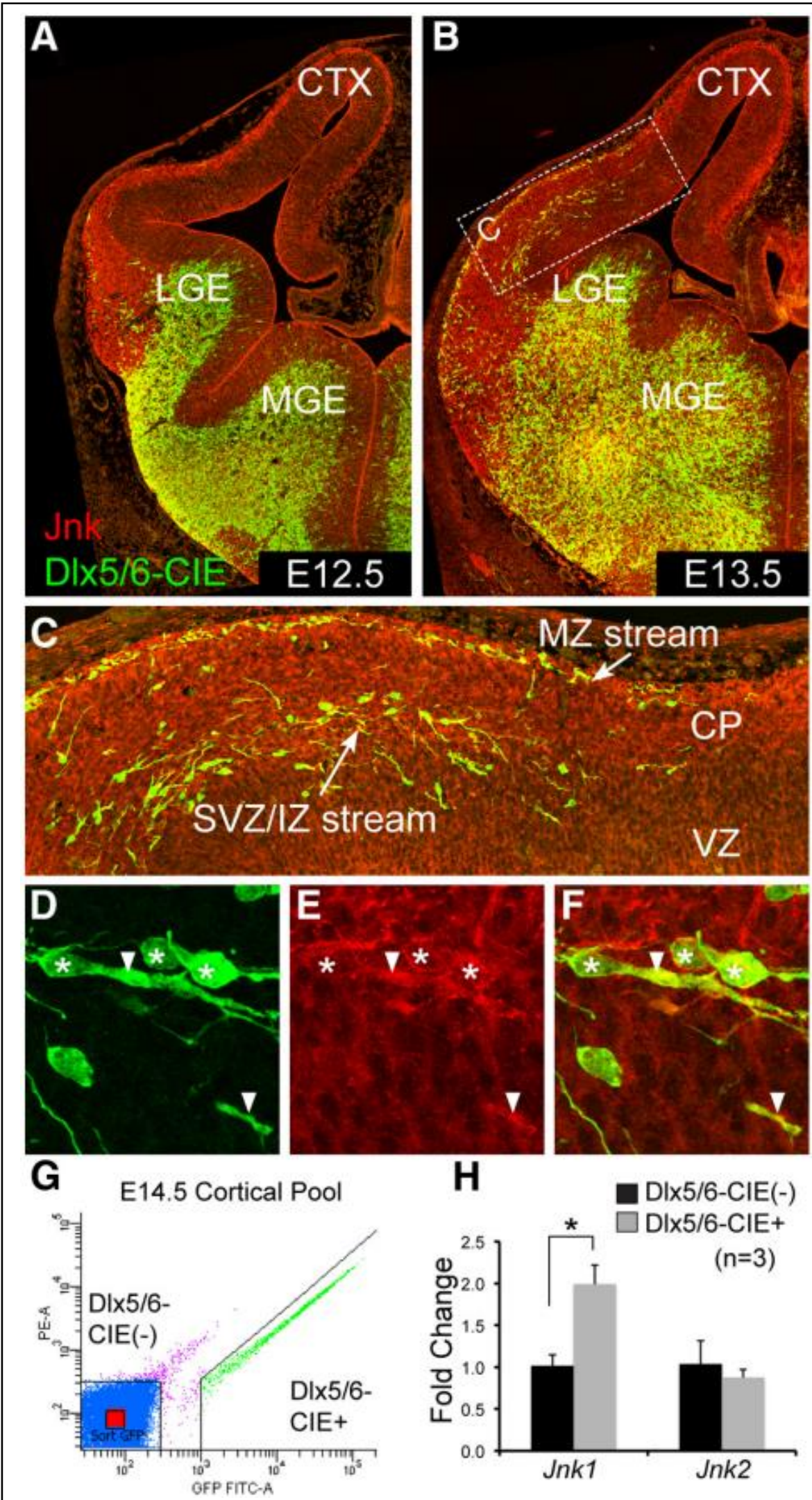
Figure 2.1: Early arriving cortical interneurons express Jnk proteins. A-F. Localization of total Jnk (red) and EGFP (green) expression in DIx5/6-CIE+ embryonic brain sections. Jnk proteins are widely expressed in the developing forebrain at E12.5 and E13.5, and are particularly enriched in post-mitotic zones. A. At E12.5, few Dlx5/6-CIE+ cortical interneurons have entered the cortex (CTX) from subcortical locations. B, C. By E13.5, many Dlx5/6-CIE+ cortical interneurons have invaded the cortical rudiment and formed streams of migratory cells in the marginal zone (MZ) and sub-ventricular zone/intermediate zone (SVZ/IZ) of the nascent cortex. D-F. DIx5/6-CIE+ interneuronal cell bodies (asterisks) and processes (arrowheads) co-localize with total Jnk protein in the E13.5 cortex. G. Separation of DIx5/6-CIE(+) and DIx5/6-CIE(-) cells from a dissociated E14.5 cortex by FACS sorting. Three cortices were independently processed for gene expression analyses by qRT-PCR. H. qRT-PCR indicates Jnk1 transcript is enriched twofold in DIx5/6-CIE $(+)$ cells compared to DIx5/6-CIE(-) cells (Student's t-test; $p=0.02$ ), whereas Jnk2 transcript is uniformly expressed in the two populations. CP, Cortical pool. 


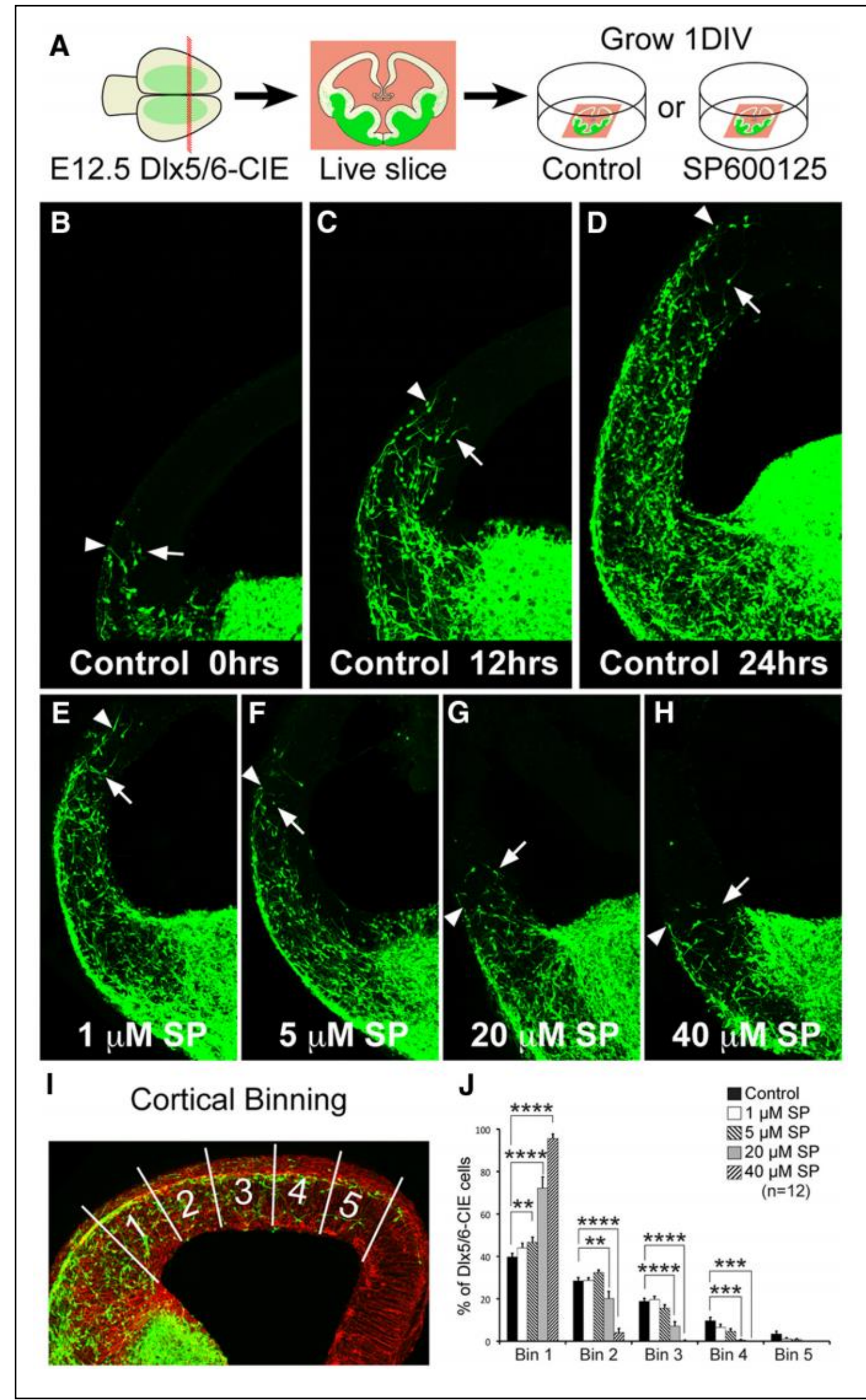


Figure 2.2: Cortical interneurons require JNK activity to enter the developing cerebral cortex. A. Schematic representation of slice culture assay. E12.5 DIx5/6-CIE+ brains are isolated, sectioned, and cultured 24 hours in control or SP600125 (SP) conditions. B-D. In control cultures, cortical interneurons robustly migrate into the cortical rudiment during the 24-hour culture period. Migratory front is marked by a flanking arrowhead (MZ region) and arrow (SVZ region) in each image. E-H. Entry of cortical interneurons into the cerebral cortex is disrupted by SP600125, a pan JNK inhibitor, in a dose-dependent fashion. I. For quantification of interneuron migration in slice cultures, cortices were segmented into 5 equidistant bins and the percentages of cortical interneurons appearing in all cortical bins were determined for each section, and averaged across all sections per treatment group. J. Dose-dependent accumulation of cortical interneurons in lateral and decline of interneurons in medial cortical bins following SP600125 treatment. Two-way ANOVA reveals statistically significant interactions between treatment and bin location $\left(F_{(16,275)}=57.5 ; p<0.0001\right)$. Differences within bins were determined post-hoc by Fisher's LSD tests $\left({ }^{* * *} p<0.0001 ;{ }^{* * *} p \leq 0.0006 ;{ }^{* *} p<0.008\right)$. 


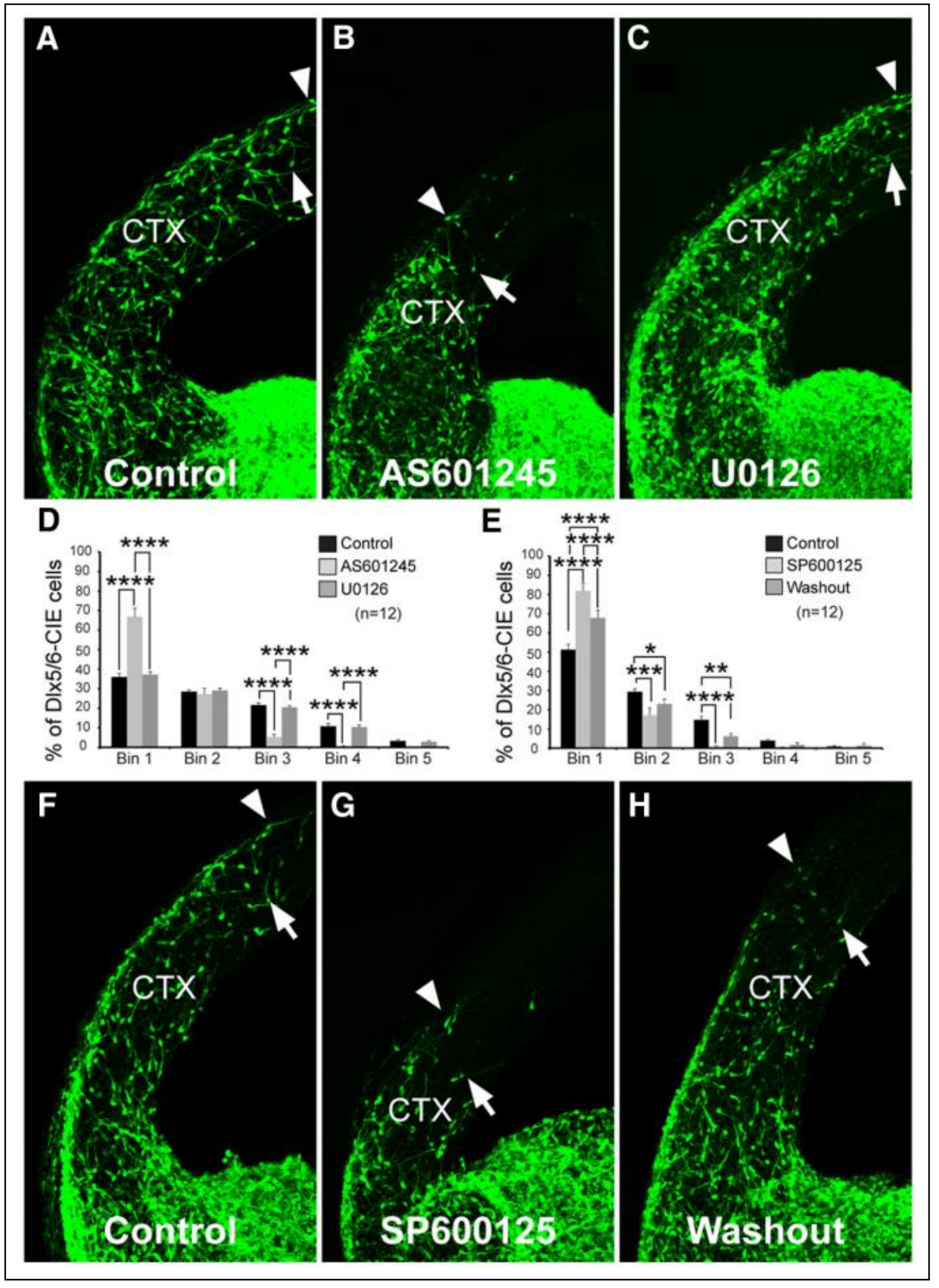


Figure 2.3: Disruption of cortical interneuron migration by pharmacological inhibition of JNK signaling is specific and reversible. A-D. An independent, pan-JNK inhibitor, AS601245 (B), diminishes interneuron entry into the cortex compared to control $(\mathbf{A})$ cultures, while blockade of Mapk signaling with the Mek1/2 inhibitor U0126 (C) does not. D. Quantification reveals significant reductions in migration for slices treated with $5 \mu \mathrm{M}$ AS601245, but no change in slices treated with $10 \mu \mathrm{M}$ U0126 (two-way ANOVA: $F_{(8,165)}=15.98 ; p<0.0001$; Fisher's LSD: $\left.{ }^{\star * * *} p<0.0001 ;{ }^{* *} p=0.0002 ;{ }^{* \star} p=0.009 ;{ }^{*} p=0.049\right)$. E-H. Cortical interneuron migration recovers after removal of $20 \mu \mathrm{M}$ SP600125. E. Quantification reveals that interneuron migration improves when $20 \mu \mathrm{M}$ SP600125 is washed out and replaced by control medium half way through the 24-hour culture period (two-way ANOVA: $F_{(8,165)}=37.56$; $p<0.0001$; Fisher's LSD: $\left.{ }^{* * * *} p<0.0001\right)$. F. Slice cultured in control medium, rinsed at 12 hours, and cultured for an additional 12 hours in control medium. G. Slice cultured in $20 \mu \mathrm{M}$ SP600125, rinsed at 12 hours, and cultured for an additional 12 hours in fresh $20 \mu \mathrm{M}$ SP600125. H. Slice cultured in $20 \mu \mathrm{M}$ SP600125, rinsed at 12 hours, and cultured for an additional 12 hours in control medium. 


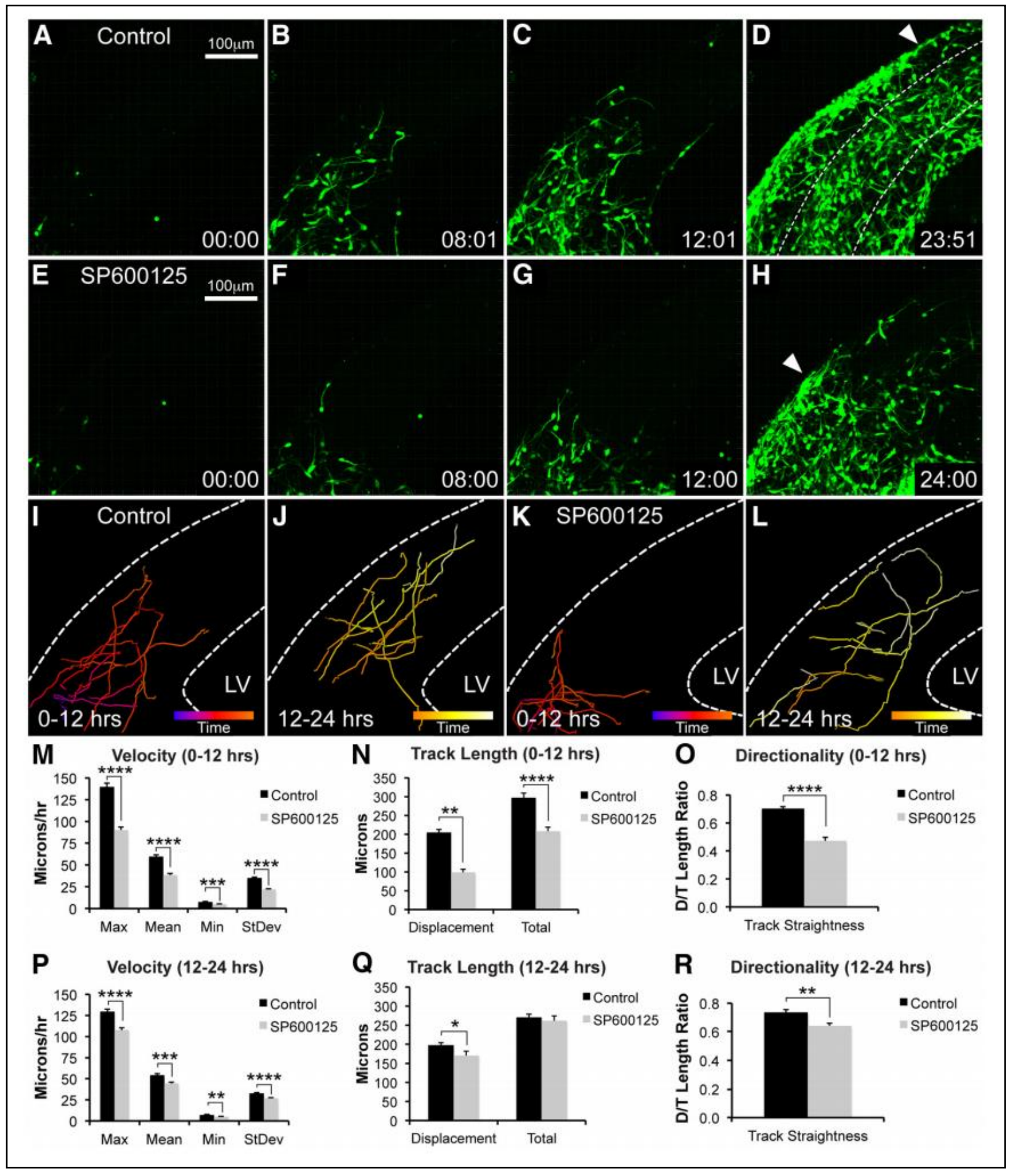

Figure 2.4: Migratory properties of cortical interneurons are perturbed following SP600125 treatment at E12.5. A-D. In control slices, DIx5/6-CIE+ interneurons robustly migrate through the cortical entry zone over a 24-hour imaging period. Cells travel in MZ region (arrowhead in D) 
and SVZ region (dashed lines in D) of the section. E-H. In slices treated with $20 \mu \mathrm{M} \mathrm{SP600125,}$ DIx5/6-CIE+ cells remain motile, but advance slowly and frequently take aberrant trajectories. Cells pile up in MZ (arrowhead in $\mathbf{H}$ ), failing to advance further. I, J. Tracks from ten individual cells in the first $12(\mathbf{I})$ and last $12(\mathrm{~J})$ hours of a control movie. Individual cell tracks are long, straight and medially directed. K, L. Tracks from 10 individual cells in the first 12 (K) and last 12 (L) hours of an inhibitor movie. Cell tracks are short, crooked, and less medially oriented in SP600125-treated slices, particularly in the first 12 hours of imaging. Tracks are pseudo-colored by time (bars in I-L). M-O. Comparison of interneuron migration in SP600125-treated and control slices during the first 12 hours of recordings. SP600125 treatment leads to statistically significant reductions in migratory velocity $(\mathbf{M})$, track length $(\mathbf{N})$, and track straightness $(\mathbf{O})$ corresponding to directionality. P-R. Comparison of interneuron migration in SP600125-treated and control slices in the last 12 hours of recordings. Interneuron migratory velocity $(\mathbf{P})$ increases in SP600125-treated slices during the second 12 hours of recording due to waning inhibitor efficacy, but remains significantly reduced from controls. Track length $(\mathbf{Q})$ of JNK-inhibited interneurons is also increased during the last 12 hours, but displacement values remain significantly altered. Directionality (R) of interneurons from SP600125-treated slices remains significantly altered in the last 12 hours. Significance levels after performing two-tailed unpaired Student's t-tests are denoted as: ${ }^{* * * *} p<0.0001,{ }^{* * *} p<0.001,{ }^{* *} p<0.01,{ }^{*} p<0.05$. 


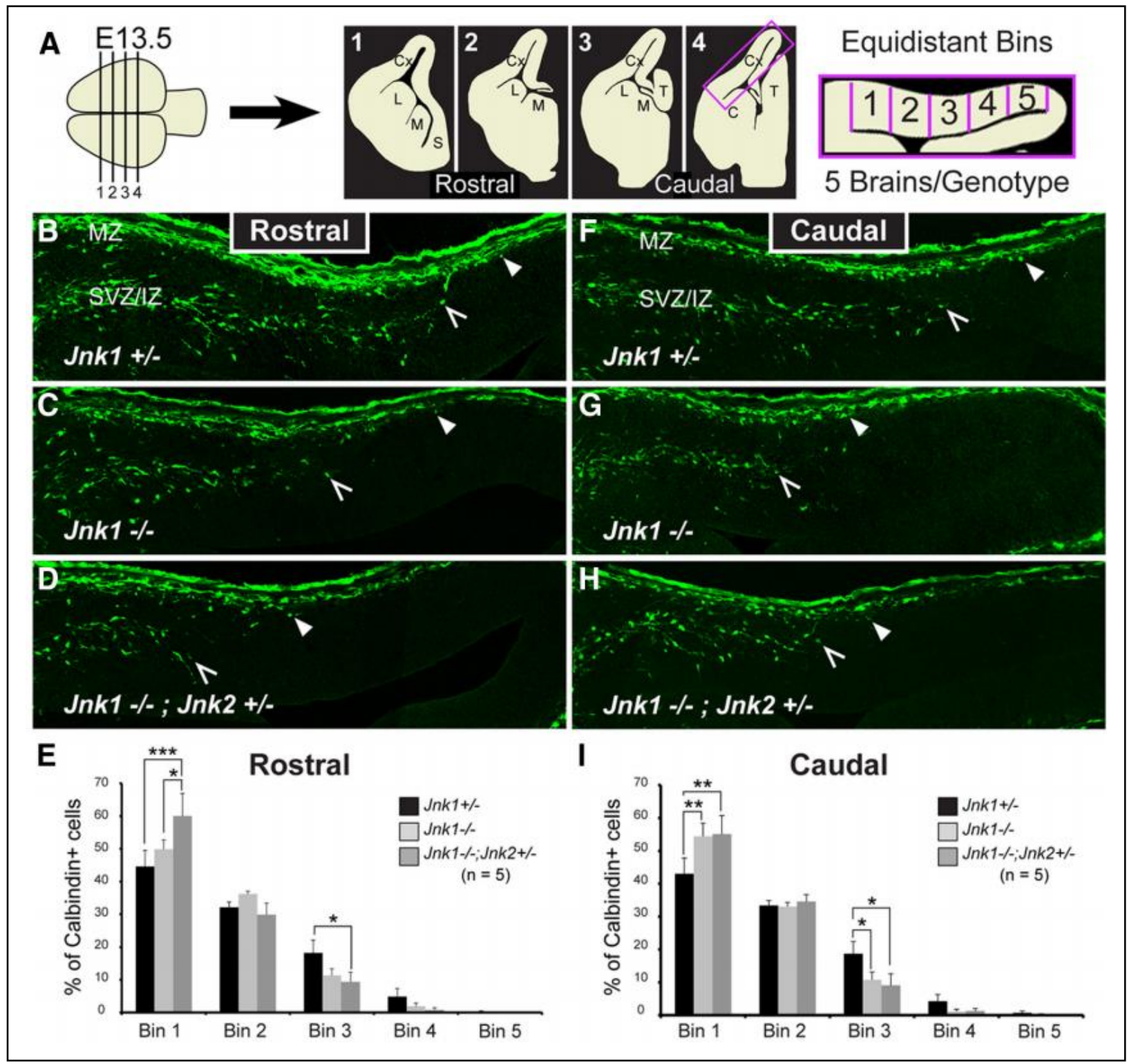

Figure 2.5: Genetic ablation of Jnk1 impairs migration of cortical interneurons in vivo. A.

Sampling strategy for mutant embryo analyses (Figures 2.5-2.7). Five E13.5 brains were serially sectioned and bilaterally sampled at four rostral-caudal locations per genotype (Cx, cortex; L, LGE; M, MGE; C, CGE; S, septum; T, thalamus). Cortices were divided into 5 equidistant bins, and the percentages of calbindin-positive cells appearing in cortical bins were determined. Data from rostral (slices 1 and 2) and caudal (slices 3 and 4) positions were analyzed separately. BE. Distribution of calbindin-positive cells in $J n k 1^{+--}(\mathbf{B}), J n k 1^{-/-}(\mathbf{C})$, and $J n k 1^{-/-} ; J n k 2^{+--}$(D) cortices 
at rostral positions. Furthest extent of interneuron progression is marked by closed (MZ) and open (SVZ/IZ) arrowheads in each image. E. Statistically significant interactions were found between genotype and bin location (two-way ANOVA: $F_{(8,60)}=2.742 ; p=0.0120$ ). Compared to $J n k 1^{+-}$controls, migration of calbindin-positive cortical interneurons was significantly impaired in Jnk1 ${ }^{-/} ;$Jnk2 ${ }^{+/}$embryos (Fisher's LSD: ${ }^{* * *} p=0.0005 ;{ }^{*} p=0.019$ in Bin 1, 0.042 in Bin 3). F-I. Distribution of calbindin-positive cells in the caudal cortex of $J n k 1^{+-}(\mathbf{F})$, Jnk $1^{-/}(\mathbf{G})$, and $J n k 1^{-/}$ ;Jnk2+/- embryonic brains (H). I. Statistically significant interactions were observed between genotype and bin location (two-way ANOVA: $F_{(8,60)}=2.463 ; p=0.0223$ ). Migration of calbindinpositive cortical interneurons was significantly impaired when Jnk ${ }^{-/}$(Fisher's LSD: ${ }^{* *} \mathrm{p}=0.005$; ${ }^{*} p<0.049$ ) and Jnk1 ${ }^{-/} ;$Jnk2 ${ }^{+/}$cortices (Fisher's LSD: ${ }^{* *} p=0.003 ;{ }^{*} p=0.017$ ) were compared to Jnk $1^{+-}$controls. 


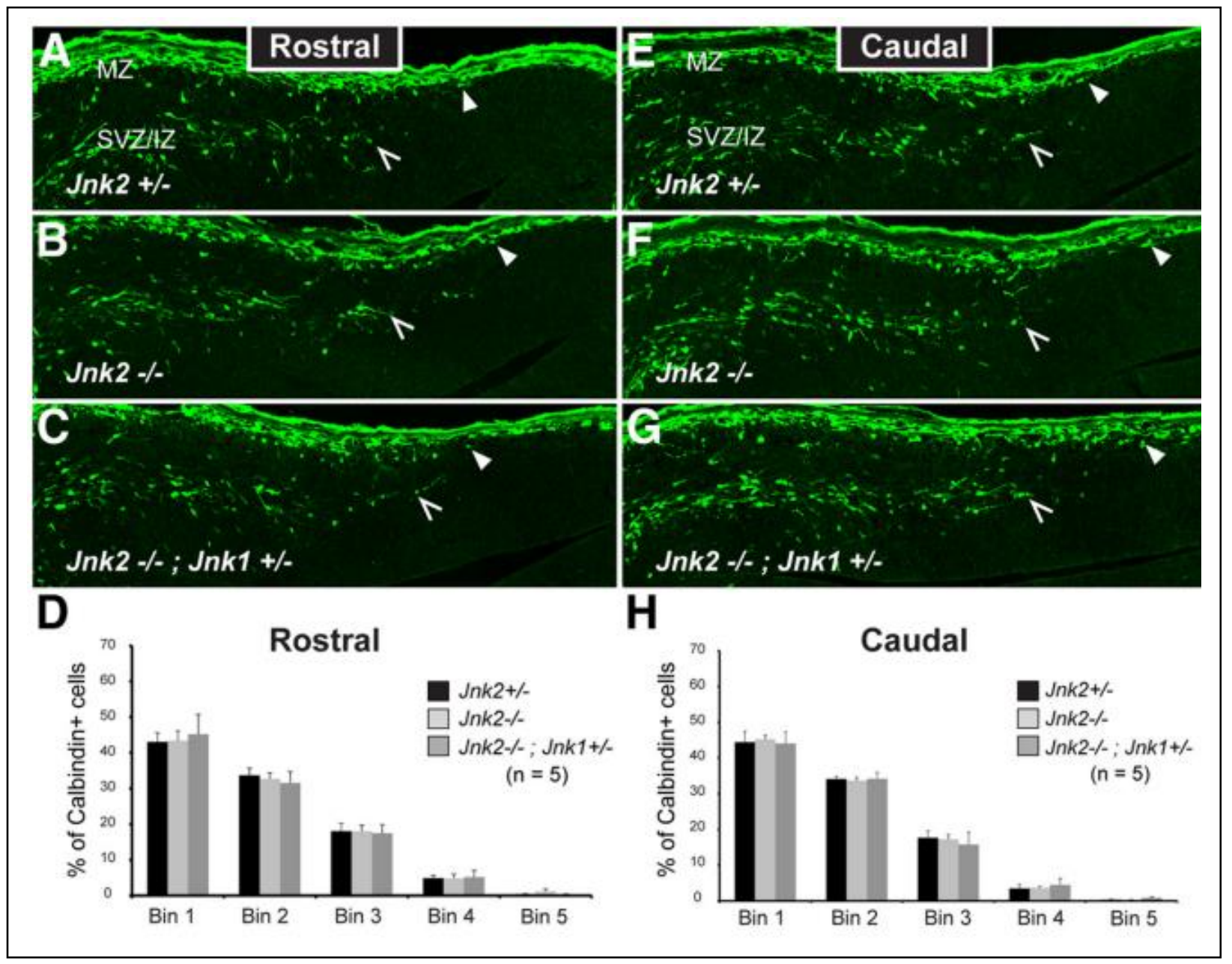

Figure 2.6: Unlike Jnk1, genetic ablation of Jnk2 does not disrupt migration of cortical interneurons in vivo. At both rostral (A-D) and caudal (E-H) levels, calbindin-positive cortical interneurons are equally distributed in $J n k 2^{+--}(\mathbf{A}, \mathbf{E}), \operatorname{Jnk} 2^{-/}(\mathbf{B}, \mathbf{F})$, and $J n k 2^{-/} ; J n k 1^{+/-}(\mathbf{C}, \mathbf{G})$ embryos. No statistically significant interactions were found between genotype and bin location at either rostral (two-way ANOVA: $F_{(8,60)}=0.131 ; p=0.998 ; D$ ) or caudal positions (two-way $\left.\operatorname{ANOVA~}_{(8,60)}=0.135 ; p=0.997 ; \mathbf{H}\right)$. 


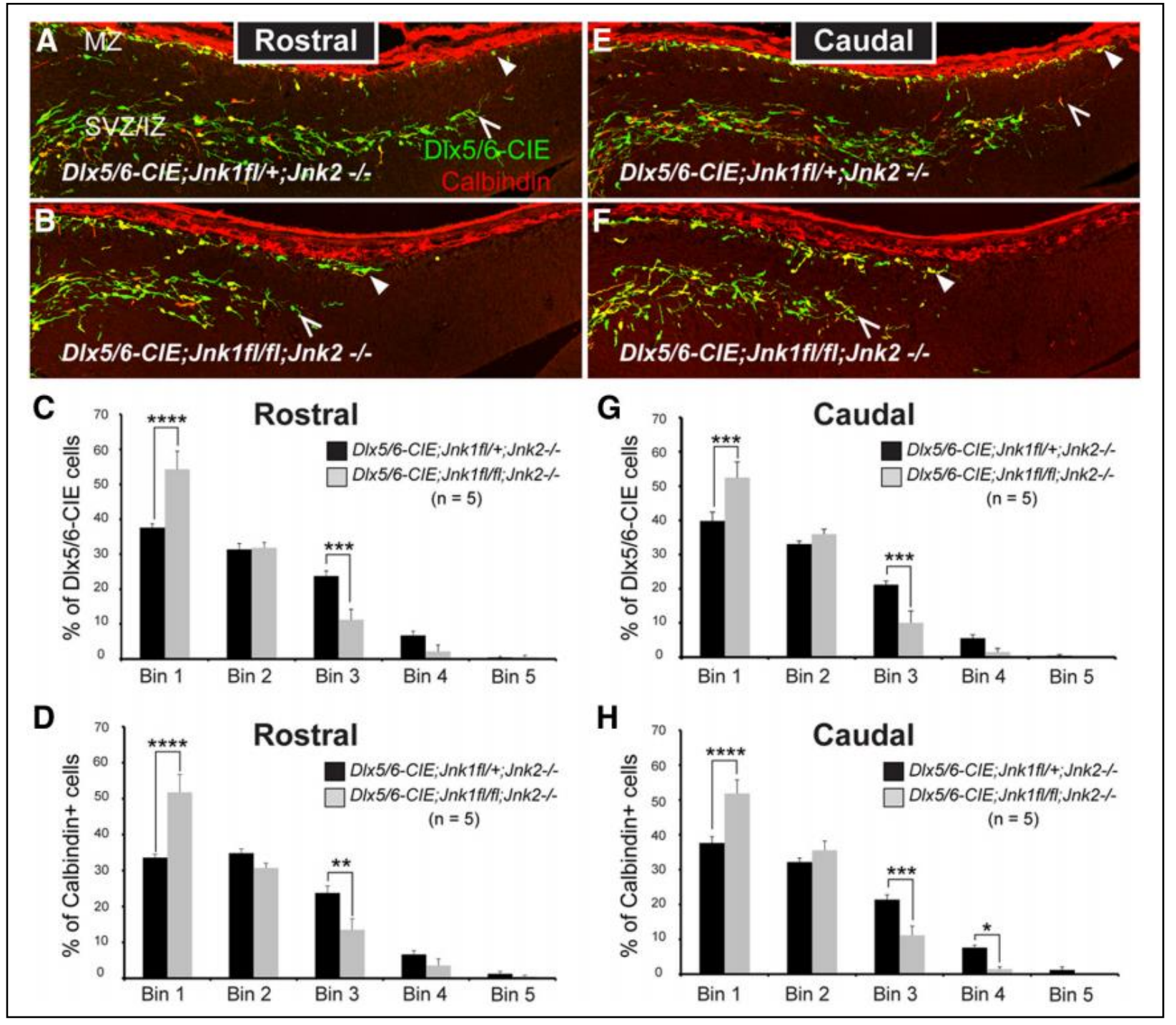

Figure 2.7: Cortical interneurons have a cell-intrinsic requirement for JNK signaling to migrate through the cortical entry zone in vivo. Jnk1 was conditionally ablated in DIx5/6-CIE+ cells of Jnk2 ${ }^{--}$embryos. A-D. Distribution of Dlx5/6-CIE+ (green) and calbindin-positive (red/yellow) cortical interneurons in the rostral cortex of DIx5/6-CIE;Jnk $1^{f / /+} ; \mathrm{Jnk} 2^{-/-}(\mathbf{A})$ and DIx5/6CIE;Jnk $1^{f / f f l} ;$ Jnk2 $2^{--}$embryonic brains (B). C, D. Statistically significant interactions were found between genotype and bin location for DIx5/6-CIE+ (two-way $\operatorname{ANOVA~F}_{(4,40)}=11.55 ; \mathrm{p}<$ $0.0001 ; \mathrm{C}$ ) and calbindin-positive cortical interneurons (two-way $\operatorname{ANOVA~}_{(4,40)}=12.42 ; \mathrm{p}<$ 0.0001 ; D). Migratory advancement of interneurons was significantly impaired in D/x5/6- 


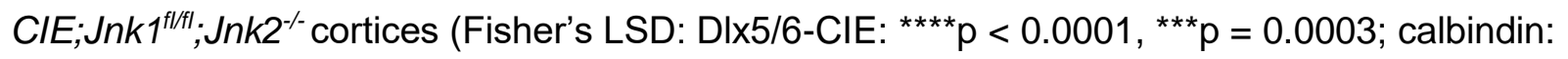
$\left.{ }^{\star * * *} p<0.0001,{ }^{* \star} p=0.002\right)$. E-H. Distribution of DIx5/6-CIE+ and calbindin-positive cortical

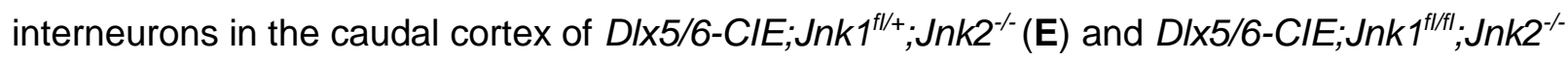
embryonic brains $(\mathbf{F})$. G, H. Statistically significant interactions were found between genotype and bin location for both DIx5/6-CIE+ (two-way ANOVA: $\left.F_{(4,40)}=8.336 ; p<0.0001\right)(\mathbf{G})$ and calbindin-positive $(H)$ cortical interneurons (two-way ANOVA: $F_{(4,40)}=11.98 ; p<0.0001$ ). Similar to rostral levels, migratory advancement of interneurons was significantly impaired at caudal levels in DIx5/6-CIE;Jnk $1^{t / / f t} ;$ Jnk2 ${ }^{-/}$cortices (Fisher's LSD: DIx5/6-CIE: ${ }^{* * *} \mathrm{p}=0.0002$ in bin1, 0.0007 in bin 3; calbindin: ${ }^{* \star *} p<0.0001,{ }^{* * *} p=0.0007,{ }^{*} p=0.029$ ). 


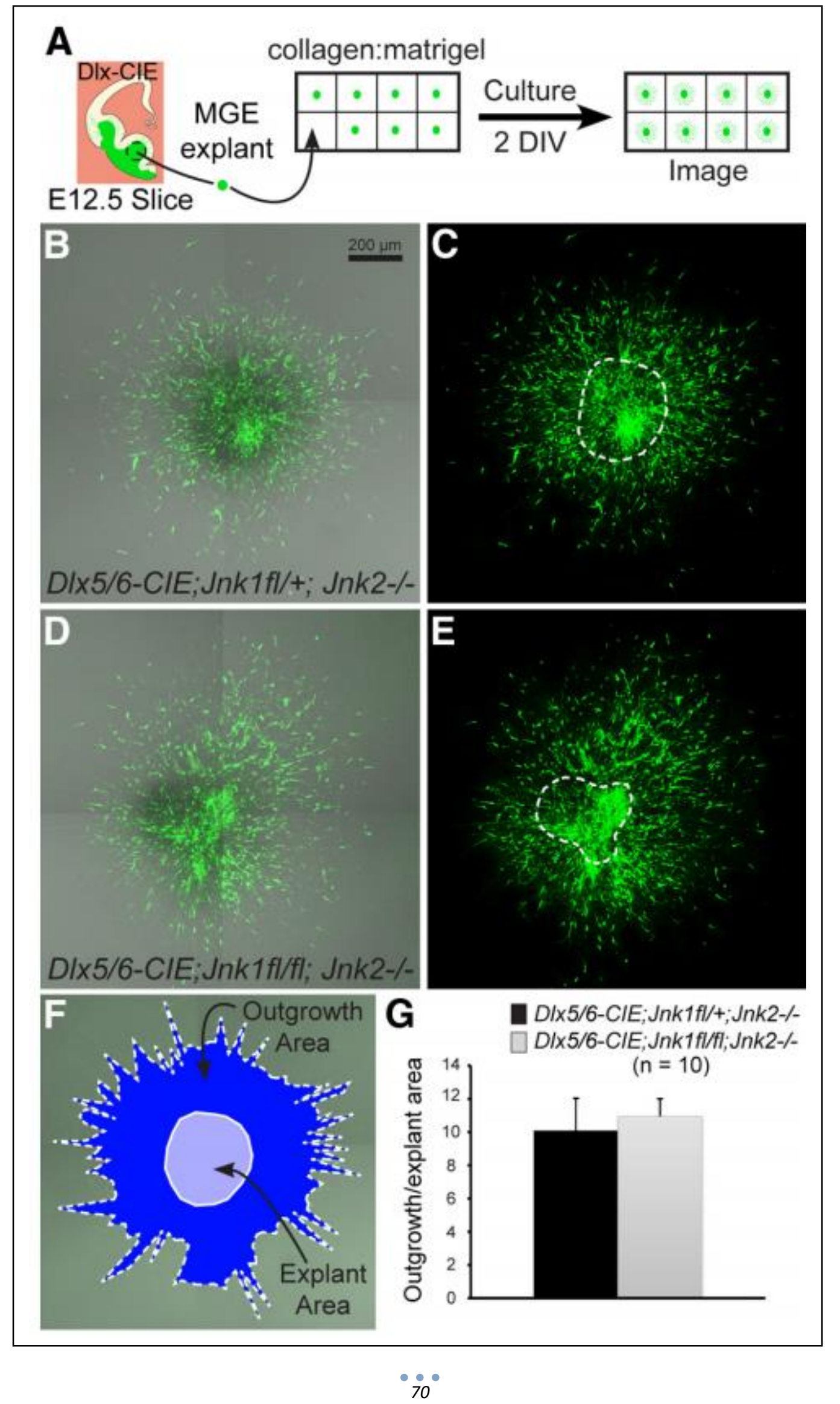


Figure 2.8: In vitro migration of cortical interneurons from explants of MGE tissue does not rely on JNK signaling. A. Illustration of experimental design (see Materials and Methods). B-C. Explant of MGE tissue isolated from an E12.5 DIx5/6-CIE;Jnk $1^{f / /+} ; \mathrm{Jnk} 2^{-/-}$embryonic brain section and cultured for 2 days in a collagen:Matrigel matrix. Interneurons have migrated from explant

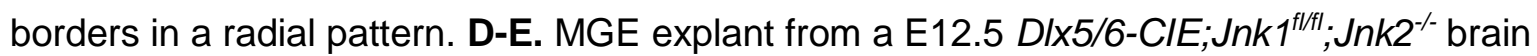
grown under identical conditions, and exhibiting a similar pattern of interneuron outgrowth. F. Areas measured from explant shown in $\mathbf{B}$ and $\mathbf{C}$, illustrating that outgrowth area (dark blue) was determined by subtracting the explant area (light blue) from the total area occupied by both explant and migrating cells. G. Plot representing mean values of normalized outgrowth area (outgrowth area/explant area) for 10 explants from each genotype. DIC images (B, D) were used to identify explant boundaries, while perimeters of migrating cells were traced from images containing the EGFP channel alone (C, E). 


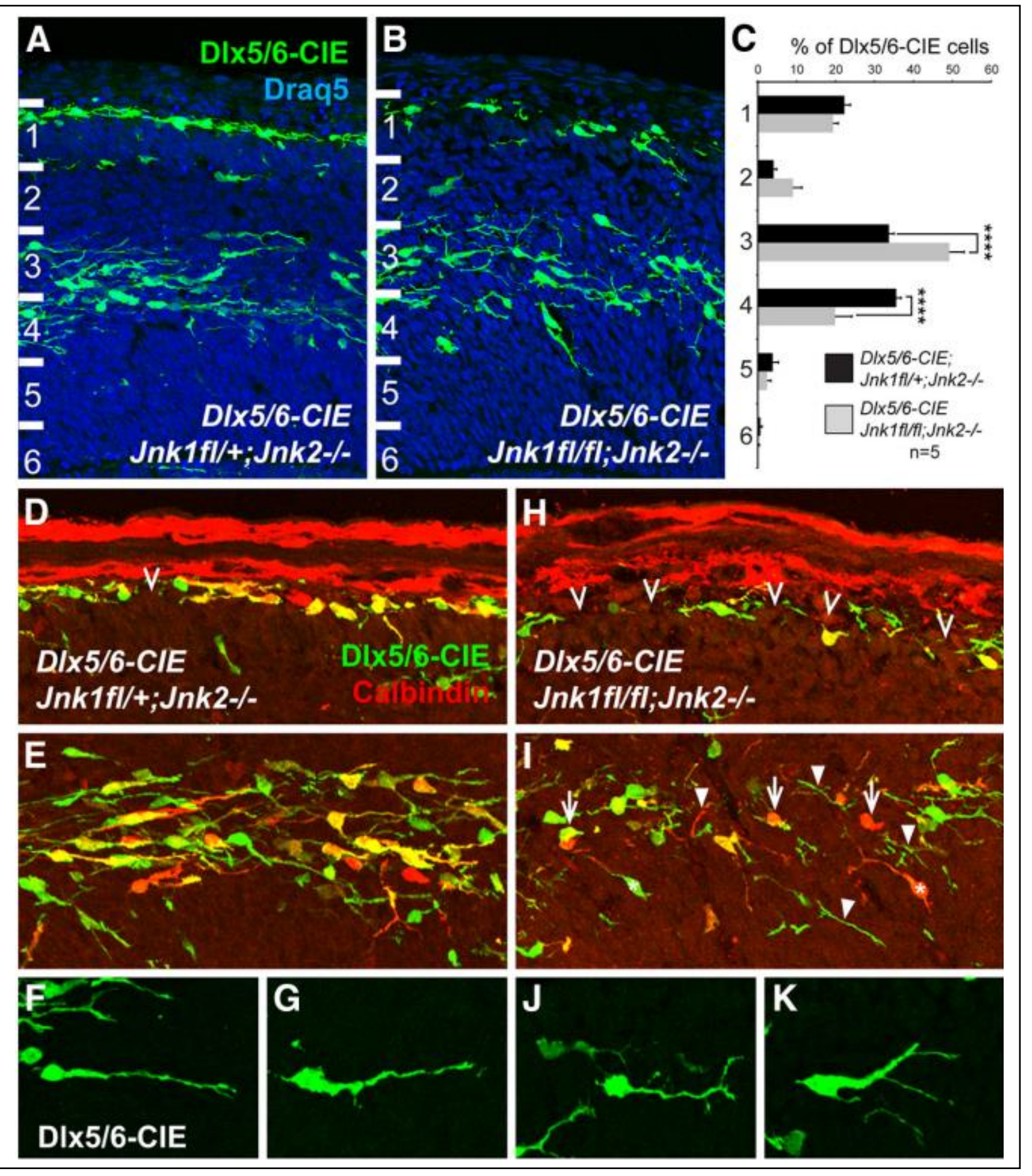

Figure 2.9: Cortical interneurons lacking Jnk1 and Jnk2 disperse from migratory streams and adopt branched morphologies in vivo. A-C. Radial distribution of Dlx5/6-ClE+ cells (green) in the lateral cortex of E13.5 DIX5/6-CIE;Jnk ${ }^{f l /+} ; J n k 2^{-/-}(\mathbf{A})$ and DIx5/6-CIE;Jnk $1^{f l / f l} ; J n k 2^{-/-}$embryos 
(B). Equivalently sized cortical probes were equidistantly binned along the radial axis (Bin 1= $\mathrm{MZ}$ - Bin $6=\mathrm{VZ}$ ) and the percentage of DIx5/6-CIE+ cells appearing in each bin was determined ( $\mathrm{n}=5$ embryos/genotype). C. Radial distribution of DIx5/6-CIE+ cells is altered in D/x5/6CIE;Jnk $1^{t / f t} ;$ J Ink2 $2^{-}$cortices (two-way ANOVA: $F_{(5,48)}=13.26 ; p<0.0001$ ). Proportionately fewer DIx5/6-CIE+ cells are located in bin 4 (SVZ region; Fisher's LSD $\left.={ }^{* * * *} p<0.0001\right)$, while more are located in bin 3 (IZ region; Fisher's LSD $\left.={ }^{* * * *} p<0.0001\right)$. D-G. Dlx5/6-CIE+ (green) and calbindin-positive cells (red/yellow) from E13.5 D/x5/6-CIE;Jnk $1^{t /+} ;$ Jnk $2^{-/}$cortices. D. Cortical interneurons within the MZ travel in a coherent stream with few gaps (open arrowhead). E. Interneurons within the cortical SVZ/IZ stream bare medially directed (image right) leading processes. F-G. Examples of isolated Dlx5/6-ClE+ cells with simple, unbranched leading processes. H-K. DIx5/6-CIE+ (green) and calbindin-positive cells (red/yellow) from E13.5 DIx5/6-CIE;Jnk $1^{f / f /} ; J n k 2^{-/}$cortices. H. Mutant MZ streams are patchy, containing many gaps (open arrowheads). I. Cortical interneurons are loosely organized within the mutant SVZ. Many interneuronal cell bodies (arrows) have no apparent leading processes, and many interneuronal processes (arrowheads) have no apparent cell bodies, suggesting many interneurons are orthogonally positioned to the plane of section. Interneurons with attached processes (asterisks) are often non-medially directed. J-K. DIx5/6-CIE+ cells from mutant cortices often have branched morphologies. Also note aberrant branching of interneurons in $\mathbf{B}$ and I compared to $\mathbf{A}$ and $\mathbf{E}$. 


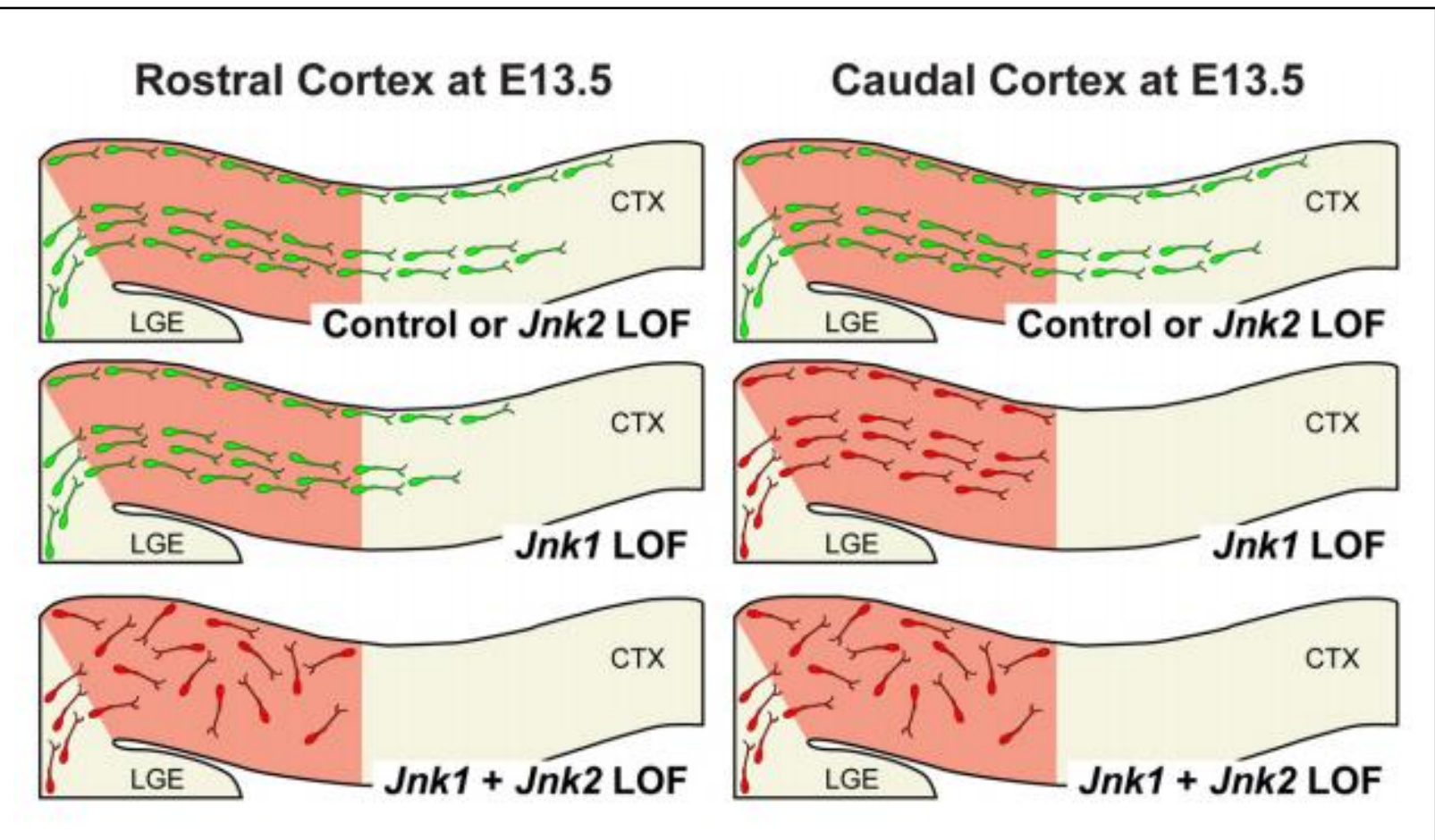

Figure 2.10: Migration of cortical interneurons through the cortical entry zone in vivo requires intact JNK signaling, largely mediated by interneuron expressed Jnk1. Schematic drawings of the cortical rudiment at E13.5; ventro-lateral is left, dorso-medial is right. Red shading represents JNK-dependent cortical "entry zone". LOF, loss of function. Control Jnk $1^{+/-}, \mathrm{Jnk} 2^{+/}$,

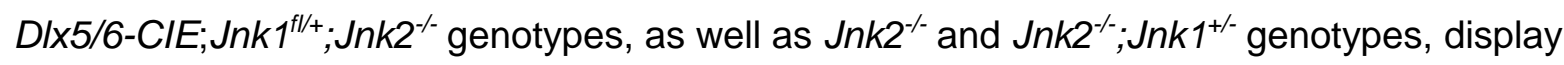
normal tangential migration at rostral (top left) and caudal (top right) cortical locations at E13.5. Cortical interneuron migration within $J n k 1^{-1-}$ embryos is diminished to statistically significant levels (red interneurons) at caudal (middle right), but not rostral locations (middle left). Jnk $1^{-1}$

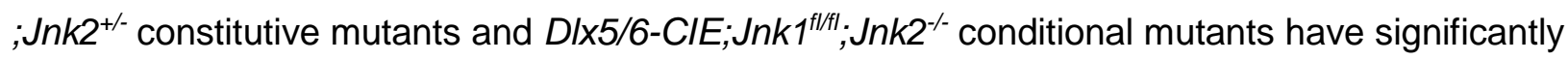
diminished migration (red interneurons) at both rostral (bottom left) and caudal locations (bottom right). Additionally, interneurons completely devoid of both Jnk1 and Jnk2 are abnormal positioned in the radial axis of the cortex, disrupting the integrity of MZ and SVZ/IZ streams, and exhibit branching anomalies. 


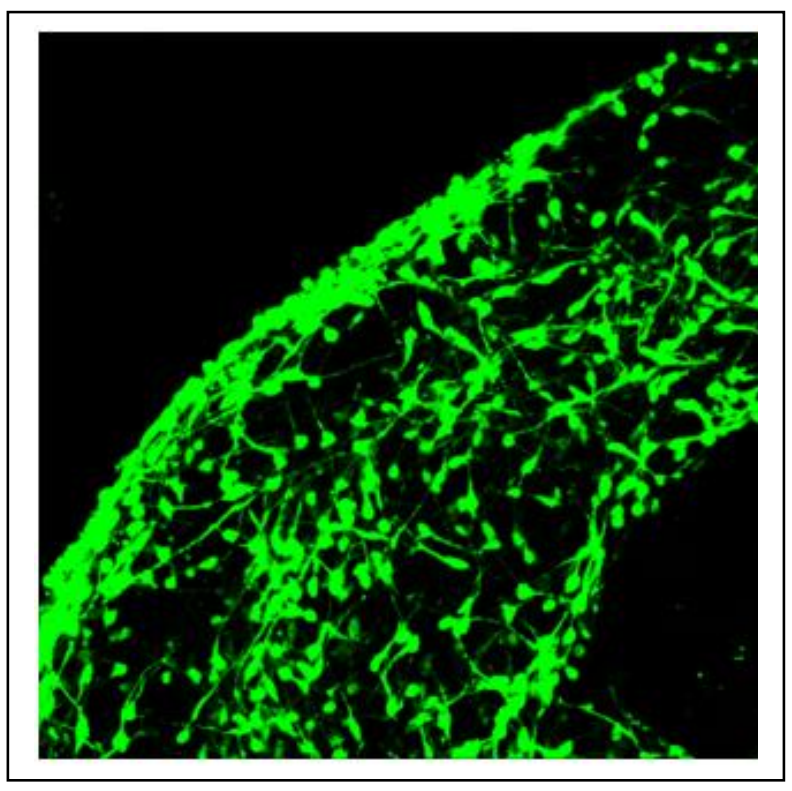

Movie 2.1. Live imaging of cortical interneuron migration through the cortical entry zone at E12.5 in a control slice (see Figure 2.4). Video clip 1. Ventro-lateral, bottom left; Dorso-medial, upper right. DIx5/6-CIE+ cells organize into migratory streams and rapidly invade the nascent cortical rudiment over the 24-hour imaging period. Video clip 2. Cell track 1 (purple spot and tail) represents a DIx5/6-CIE + cell tracked during the first 12-hour imaging period. Cell 1 maintains a medially directed trajectory, but shifts from SVZ to VZ regions of the slice during the tracking period. Video clip 3. Cell track 2 (red spot and tail) represents a Dlx5/6-CIE+ cell tracked during the last 12-hour imaging period. Cell 2 maintains a long, medially directed trajectory, but shifts positions within the SVZ/IZ stream during the tracking period. Spots mark tracked cells, and tails represent where the cell traveled over the last hour of time. 


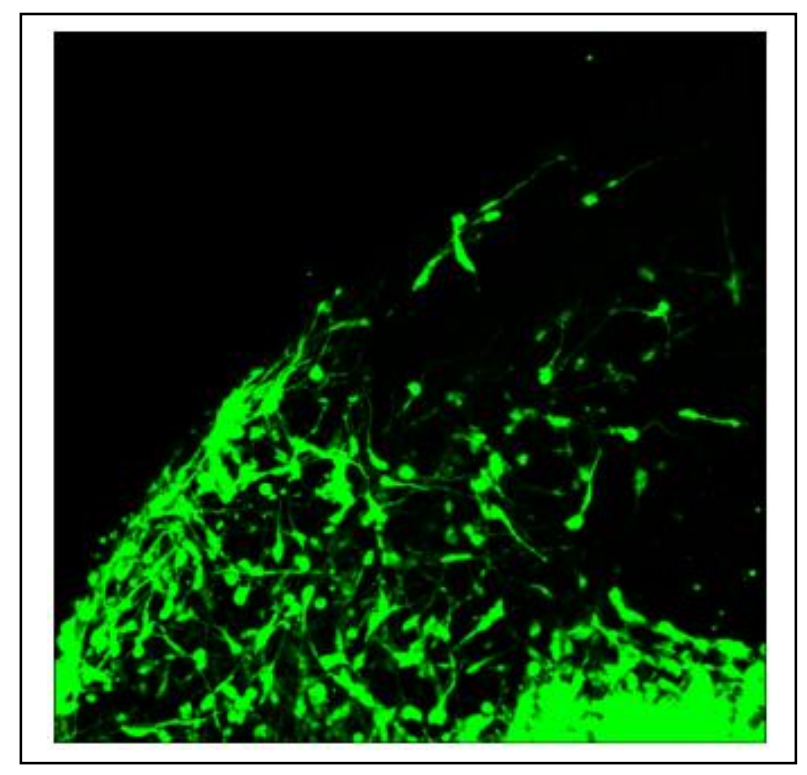

Movie 2.2. Live imaging of cortical interneuron migration through the cortical entry zone at E12.5 in an SP600125-treated slice (see Figure 2.4). Video clip 1. Ventro-lateral, bottom left; dorso-medial, upper right. DIx5/6-CIE+ cells tentatively migrate into the cortical entry zone during the first 12 hours of recording, often migrating slowly into the cortex only to reverse directions and leave. Migration improves during the last 12 hours of recording, but many cells pile up in the $M Z$ stream or spread out in the SVZ/IZ region without advancing past the cortical entry zone. Video clip 2. Cell track 1 (purple spot and tail) represents a Dlx5/6-CIE+ cell tracked during the first 12-hour imaging period. Cell 1 migrates a short distance medially, traveling from the SVZ/IZ to the MZ regions of the slice, only to stall, turn, and migrate back towards the cortico-striatal border. Video clip 3. Cell track 2 (red spot and tail) represents a Dlx5/6-CIE + cell tracked during the last 12-hour imaging period. Cell 2 migrates in a radial trajectory, traveling from the $\mathrm{VZ}$ to the $\mathrm{MZ}$ region of the slice, only to turn and migrate laterally, like Cell 1. Spots mark tracked cells, and tails represent where the cell traveled over the last hour of time. 


\title{
Chapter 3: JNK Signaling Preserves Cortical Interneuron Stream Integrity and Radial Organization of the Cortical Plate
}

\author{
Abigail K Myers ${ }^{1,2,4}$, Jessica G Clemente ${ }^{1,2,4}$, Skye E Hickling ${ }^{1,3,4}$, Katie Padgett ${ }^{4}$, John Snow ${ }^{4}$, \\ and Eric S Tucker ${ }^{1,4}$ \\ ${ }^{1}$ Department of Neurobiology and Anatomy, ${ }^{2}$ Neuroscience Graduate Program, ${ }^{3}$ Biochemistry \\ Graduate Program, ${ }^{4}$ Blanchette Rockefeller Neurosciences Institute, West Virginia Univ. Sch. of \\ Med., Morgantown, WV 26506
}




\section{Abstract}

The precise migration of cortical interneurons from the ganglionic eminences to the cerebral cortex is essential for cortical circuit formation and prevention of severe neurodevelopmental disorders like autism, schizophrenia, and epilepsy. Cortical interneurons navigate the developing cerebral cortex utilizing well-known molecular guidance cues, however the intracellular signaling pathways that coordinate their directed migration are not well understood. Recently, we identified the c-Jun N-terminal kinase (JNK) pathway as an important regulator of cortical interneuron migration, where it mediates their advancement into the cortical entry zone and proper assembly of migratory streams during early stages of corticogenesis. In the current study, we demonstrate a vital role for JNK signaling as cortical interneurons transition from tangential to radial migration. Pharmacological inhibition of JNK signaling in ex-vivo slice cultures caused cortical interneurons to evacuate from migratory streams and precociously enter the cortical plate. Cortical interneurons partially reassemble into migratory streams after removal of the pharmacological blockade, suggesting that migratory stream integrity depends on intact JNK signaling. Live imaging of JNK-inhibited ex-vivo slices demonstrated increased diagonal and radial trajectories of interneurons evacuating from the subventricular zone stream and increased time spent in the cortical plate upon arrival. Similarly, complete genetic loss of JNK signaling from cortical interneurons in ex-vivo slice cultures leads to dispersion of cortical interneurons from the SVZ stream and subsequent filling in of the cortical plate. Evaluation of $J n k 1^{t / f l t} ; J n k 2^{-\sim} ; J n k 3^{-\sim} ; D / x-C I E$ cortices at embryonic day (E) 15.5 revealed interneuron clusters within the entry zone of the cortex and less unified subplate and SVZ streams. Time-lapse

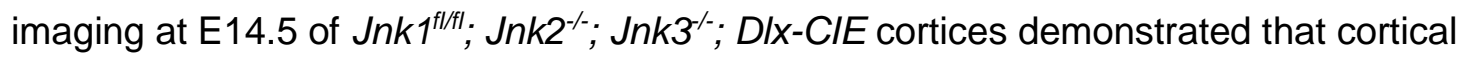
interneurons escaping the MZ stream trigger the formation of interneuron clusters in the cortical plate. Further, evaluation of underlying radial glial scaffolding suggest that the clusters of cortical interneurons may form in conjunction with disruption and detachment of radial glial cell 
processes from the pial surface. These data suggest that JNK is important for mediating the tangential to radial transition of migrating cortical interneurons and could be vital for the development of the underlying cortical architecture. 


\section{Introduction}

Proper formation of inhibitory interneuron circuitry within the developing cortex is imperative for normal cortical function, including the prevention of over-excitation. Even minor disruptions to interneuron distribution and wiring in the cerebral cortex are suspected to cause common neurological disorders like autism, schizophrenia, and epilepsy (Meencke and Veith, 1992; Meechan et al., 2012; Volk et al., 2015). Cortical circuit malfunction can result from a variety of causes; however, early migratory deficits can have a profound impact on cortical circuitry by impairing the final placement and function of cortical interneurons.

Post-mitotic cortical interneurons travel tangentially from the medial and caudal ganglionic eminences in the ventral forebrain by deciphering molecular cues to form and migrate in streams (Nery et al., 2002; Xu et al., 2004; Miyoshi et al., 2010). As development proceeds, cortical interneurons transition from tangential to radial migration to enter the cortical plate where they will layer with excitatory neurons to form the layers of the mature cerebral cortex (Miyoshi and Fishell, 2011). The mechanism by which cortical interneurons mediate this tangential to radial switch appears to be multi-factoral (Chédotal and Rijli, 2009; Elias et al., 2010; Baudoin et al., 2012). Recent studies have attributed the tangential to radial switch to the expression of connexin43 (Elias et al., 2010), the chemokine SDF-1/Cxcl12 and its receptors Cxcr4/Cxcr7 (Sánchez-Alcañiz et al., 2011; Wang et al., 2011), the morphogen SHH (Baudoin et al., 2012)), and regulation of cellular machinery through the primary cilium (Baudoin et al., 2012; Higginbotham et al., 2012). To date, the intracellular signaling pathways regulating the tangential progression of cortical interneurons in migratory streams and the timing of migratory stream exit are largely unknown.

The c-Jun N-terminal kinase pathway includes upstream activators (MKK4 and MKK7) and downstream targets (DCX, Map1B) (Gdalyahu et al., 2004; Kawauchi et al., 2005; Jin et al., 2010; Coffey, 2014). The terminal kinase is made up of 3 Jnk genes: Jnk1, Jnk2, and Jnk3 and 
known to regulate migration through the dynamic remodeling of cell cyto-architecture (Gupta et al., 1996; Gdalyahu et al., 2004; Westerlund et al., 2011; Björkblom et al., 2012). JNK signaling activates regulatory proteins such as doublecortin (Gdalyahu et al., 2004), Map1b (Chang et al., 2003), connexin43 (Barker and Gourdie, 2002) to influence cellular dynamics. Additionally, it mediates guided migration through chemokine receptors such as CXCR4 (Serrati et al., 2008; Zhe et al., 2012)) within different cell types to affect migration (Friocourt et al., 2007; Elias et al., 2010; Sánchez-Alcañiz et al., 2011; Wang et al., 2011). Therefore, deficits in JNK signaling could have implications in perturbing cortical interneuron orientation in migratory streams and their transitions from tangential to radial modes of migration (López-Bendito et al., 2008; Elias et al., 2010; Lysko et al., 2011, 2014; Sánchez-Alcañiz et al., 2011). Since our previous research showed that cortical interneurons require JNK signaling for their initial entry into the cerebral cortex and their organization within migratory streams between E12.5 and E13.5 of mouse development (Myers et al., 2014), we next considered whether JNK signaling was also required at later stages of cortical interneuron migration.

In the current study, we use pharmacological inhibition of JNK signaling in ex vivo slice cultures and in vivo deletion of the Jnk1, Jnk2, and Jnk3 genes from interneurons to investigate the role of JNK signaling in the tangential progression of cortical interneurons in migratory streams and the timing of migratory stream departure. Our findings suggest that JNK signaling is required for the maintenance of migratory streams and that inhibition of JNK signaling may facilitate the switch from tangential to radial modes of migration in order to promote the infiltration of cortical interneurons into the nascent cortical plate. 


\section{Materials and Methods}

\section{$\underline{\text { Mice }}$}

All mouse procedures were completed in accordance to the West Virginia Animal Care and Use Committee. CF-1 dams were mated to DIx5/6-Cre-IRES-EGFP (DIx5/6-CIE) males to achieve time pregnancies at embryonic day 14.5 (E14.5) for our slice culture experiments. C57 Jnk ${ }^{f / f t}$;

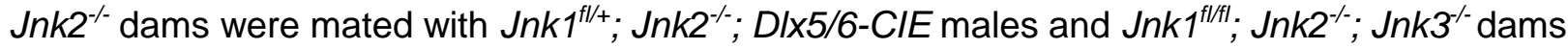
were mated $J n k 1^{f l+} ; J n k 2^{-\alpha} ; J n k 3^{+/-}$; DIX5/6-CIE males with to achieve time pregnancies at E15.5. The day of vaginal plug was embryonic day 0.5 .

\section{Slice Culture for Dosage Experiment and Washout Experiments}

The day prior to collecting the embryos, a six well plate of transwell membranes were coated with a solution of poly-I-lysine and laminin. On the experimental day, the transwell membranes were rinsed with sterile water and left to dry. DIx5/6-CIE positive embryos were collected at embryonic day 14.5 (E14.5). The embryonic brain was removed from the embryo. All brains were embedded in $3 \%$ agarose and sectioned into $300 \mu \mathrm{M}$ slices on a Leica vibratome. Slice culture media containing either Dimethyl Sulfoxide (DMSO), or a $20 \mu \mathrm{M}$ or $40 \mu \mathrm{M}$ concentration of the SP600125 inhibitor was added beneath the transwell membranes. Slices were placed on a transwell membrane and cultured in an incubator maintained at $37^{\circ} \mathrm{C}$ with $5 \% \mathrm{CO}_{2}$. All slices were fixed with $4 \%$ paraformaldehyde.

\section{Frozen Embedding of Cultured Sections}

Post fixation with 4\% paraformaldehyde, slices were transferred through a sucrose gradient $(10 \%, 20 \%$, and $30 \%)$ over three days by switching to the next consecutive percentage after a day's time. Slices were embedded in agar sucrose to help free them from the transwell membrane. The agar sucrose embedded slices were transferred to a silicon mold and embedded in Tissue Freezing Medium. The slice embedded in the silicon mold was frozen by 
plunging it into 2-Methylbutane chilled by liquid nitrogen. The frozen slices were then stored at 80 degrees.

\section{Cryosectioning and Immunocytochemistry}

Frozen slices were sectioned at $12 \mu \mathrm{m}$ on a Leica cryostat. Sections were laid down on to Superfrost Plus slides. Slides were painted with a hydrophobic barrier pen. 1x phosphate buffer saline (1xPBS) was added to each slide to rehydrate the processed sections. Permeabilization solution with $5 \%$ normal goat serum was added to the slides to block the sections. Rabbit anti Tbr1 (1:250) and Chicken anti GFP (1:1500; Abcam) primary antibodies labeled the developing cortical plate and cortical interneurons respectively. Secondary antibodies, goat anti-rabbit 546 and goat anti-chicken 488, were used to fluorescently label the primary antibodies. A nuclear counterstain was added to help identify cortical interneurons and assess cellular health.

\section{Live Imaging}

Live embryonic brain slices were processed the same as for the fixed preparations above. Slices were cultured on a transwell membrane in a glass bottom dish in either control (DMSO) or $20 \mu \mathrm{M}$ concentration of the SP600125 inhibitor. Slices were incubated at $37^{\circ} \mathrm{C}$ at $5 \% \mathrm{CO}_{2}$ and imaged on a Zeiss 710 confocal microscope with a 20x neofluar objective for 12 hours.

\section{Quantification for Dosage and Washout Experiments}

Images were cropped to $600 \times 1500$. The cropped images were binned into 10 equidistant bins. Cortical interneurons were counted using the count tool in Adobe Photoshop. The angles of the leading processes were determined by adding a protractor layer onto the image in Adobe Photoshop. Angle 0 degrees was aligned to the closest bin line. 


\section{Quantification for Live Imaging Experiments}

Movies were analyzed using the Imaris software (Bitplane). Cortical interneurons were tracked from a lateral, middle, and medial region of the subventricular zone (SVZ) stream. One box was created in the cortical plate region to assess the movement of cells in this region overtime. Cortical interneurons were tracked for at least 2 hours using the Spots feature. Cell velocity was exported from the software. The leading process of the cortical interneurons was used to assess direction. Cell direction was noted as either tangential, diagonal, or radial. A numerical score was given to each direction $(0,0.5$, or 1 respectively) and the number of transitions for each of the cells was noted. An average of 10 cells were tracked per movie 5 movies were tracked from each condition.

\section{Results}

\section{JNK signaling maintains the integrity of cortical interneuron migratory streams}

Cortical interneurons initially travel from lateral to medial locations within the developing cerebral cortex in tangentially oriented migratory streams located in the subventricular/intermediate zone (SVZ/IZ), subplate, and marginal zone (MZ), but as development proceeds, interneurons switch from tangential to diagonal or radial modes of migration in order to enter the nascent cortical plate (Wichterle et al., 2001). Although extracellular cues underlying migratory stream maintenance (Wang et al., 2011; Baudoin et al., 2012; Li et al., 2012) and cell-cell adhesive cues mediating the tangential to radial switch (Elias et al., 2010) have been identified, intracellular signaling pathways operating downstream of guidance receptors to orchestrate this complex change in migratory behavior are poorly understood. Since we previously identified a critical role for c-Jun N-terminal kinase (JNK) signaling in promoting the initial entry of cortical interneurons into the cortical rudiment and preserving their tangential orientation within migratory streams (Myers et al., 2014), we 
hypothesized that JNK may facilitate later stages of cortical interneuron migration as well. In order to determine if JNK signaling is required to maintain the integrity of pre-formed streams of migratory cortical interneurons, we cultured live-vibratome sections from embryonic day 14.5 (E14.5) DIx5/6-Cre-Ires-EGFP positive (DIx-CIE+) brains in either control or JNK inhibited conditions for 12 hours. JNK inhibition was attained by using SP600125 (Bennett et al., 2001), a pan-JNK inhibitor, at $20 \mu \mathrm{M}$ or $40 \mu \mathrm{M}$; concentrations that delayed or prevented the entry of interneurons into the cerebral cortex between E12.5 and E13.5 of mouse development (Myers et al., 2014). Cortical interneuron distribution was analyzed in two regions of the cerebral cortex: the cortical entry zone and the leading edge of interneuron migratory streams (Figure 1B). Stream dispersion was measured by dividing each region into 10 equidistant bins from the pial to ventricular surfaces and assessing the distribution of cortical interneurons across the radial axis of the cortical wall.

At E14.5, interneurons in the cortical rudiment are predominantly found traveling in robust, organized streams within the marginal zone (MZ) and subventricular zone (SVZ). As expected, $\mathrm{MZ}$ and SVZ streams were preserved in slices that were cultured for 12 hours in control conditions at both the entry zone and the leading edge of cortical interneuron migration (Figures 3.1A-C). JNK-inhibited slices, however, showed disbanding of cortical interneurons from migratory streams and precocious filling in of the cortical wall (arrows) after treatment with 20 $\mu \mathrm{M}$ (Figures 3.1D-F) and $40 \mu \mathrm{M}$ SP600125 (Figures 3.1G-I). Moreover, interneuron stream disbanding was most prominent in medial cortical regions (Figures 3.1C, F, I). Quantification of interneuron distribution at the laterally positioned entry zone showed a trend for interneuron dispersion from the SVZ and MZ streams (Figure 3.1J, upper graph), but this trend did not reach statistical significance. When interneuron distribution was quantified at the medially positioned leading edge, however, robust differences were found between control and SP600125-treated slices. Disruption to the marginal zone and subventricular zone streams in JNK-inhibited slices 
resulted in significant alterations in the radial distribution of cortical interneurons in the cortical wall (Two-way ANOVA $\left.F_{(18,120)}=3.582 ; p<0.0001\right)$. Post-hoc analyses showed statistically significant decreases of cortical interneurons traveling in the marginal zone (Fisher's LSD test: $20 \mu \mathrm{M}$, Bin $1 \mathrm{p}=0.0344 ; 40 \mu \mathrm{M}$, Bin $1 \mathrm{p}=0.0002$ ), intermediate zone (Fisher's LSD test: $20 \mu \mathrm{M}$, Bin $4 \mathrm{p}=0.0005$ ), and subventricular zone (Fisher's LSD test: $20 \mu \mathrm{M}$, Bin $5 \mathrm{p}=0.0039 ; 40 \mu \mathrm{M}$, Bin $5 p=0.0445$ ), and a significant increase of cortical interneurons in the ventricular zone (Fisher's LSD test: $20 \mu \mathrm{M}$, Bin $8 \mathrm{p}=0.0217 ; 40 \mu \mathrm{M}$, Bin $9 \mathrm{p}=0.0312$; Figure 3.1J, lower graph). More interneurons were also found in the nascent cortical plate of JNK inhibited slices, further distinguishing them from controls.

In addition to radial displacement, we quantified the orientations of interneuronal leading processes to assess the trajectory of cortical interneurons after 12 hours of JNK inhibition. Angles of leading processes were measured using a digital protractor that was aligned tangentially to the cortical wall with zero degrees always pointing towards the dorsal-medially positioned cortical arch. Interneurons with clearly distinguishable leading processes were sampled throughout the entire medial-lateral extent of the cortical rudiment. Cortical interneurons in control slices maintained a tangential orientation with leading processes angled between 0-30 degrees (Figure 3.1K, green traces) in the cortical plate (99/200 cells), subventricular zone (121/254 cells), and ventricular zone (7/16 cells). Analysis of leading processes of cortical interneurons in the SP600125-treated slices revealed a dose dependent decrease in tangentially oriented cortical interneurons in the cortical plate (20 $\mu \mathrm{M}: 57 / 178$ cells; 40 $\mu \mathrm{M}: 82 / 256$ cells) subventricular zone ( $20 \mu \mathrm{M} 80 / 250$ cells; $40 \mu \mathrm{M}$ 57/237 cells), and ventricular zone (20 $\mu \mathrm{M}$ : 11/41 cells; $40 \mu \mathrm{M}$ : 5/55 cells; Figure $3.1 \mathrm{~K}$, blue traces-20 $\mu \mathrm{M}$ and red traces $-40 \mu \mathrm{M})$. The decreased frequencies of tangentially oriented (0-30 degrees) cortical interneurons in SP600125-treated slices were statistically significant in both the subventricular zone (Chi Square Analysis: $20 \mu \mathrm{M}$ : $\mathrm{p}=0.0004 ; 40 \mu \mathrm{M}$ : $\mathrm{p}<0.0001$ ) and cortical plate (Chi Square 
Analysis: $20 \mu \mathrm{M}$ : $\mathrm{p}=0.0008 ; 40 \mu \mathrm{M}: \mathrm{p}=0.0002$ ). Many cortical interneurons in SP600125-treated conditions displayed a wider array of angles, with numerous cortical interneurons angled opposite of their correct trajectory, and others angled toward the cortical plate and ventricular zone regions. Again, increased frequencies of cortical interneurons with aberrant orientations in SP600125-treated slices were statistically significant in the cortical plate regions (Figure 3.1K) Collectively, our data suggest that JNK inhibition leads to the departure of cortical interneurons from migratory streams, and that migratory stream integrity might differ in its requirement for JNK signaling along the medial-lateral axis. Interneuron streams in the medial cortex appear more sensitive to pharmacological inhibition of JNK signaling than in the lateral cortex.

\section{Stream integrity can be partially restored after disruption of JNK signaling}

To determine if the stream integrity that was lost in the SP600125 pharmacologically treated tissue could be recovered through restoration of JNK signaling, we performed a washout experiment on Dlx-ClE+ slices. Slices were cultured in-vitro for 12 hours in control media, or media treated with $20 \mu \mathrm{M}$ or $40 \mu \mathrm{M}$ SP600125. After rinsing with control media at 12 hours, half of the cultures initially treated with SP600125 were replaced with media containing the same concentration of SP600125, while the other half of SP600125-treated cultures were replaced with control media. All slice cultures were grown for an additional 12 hours.

Slices treated with $20 \mu \mathrm{M}$ or $40 \mu \mathrm{M}$ SP600125 for 24 hours contained cortical interneurons that dispersed from the subventricular zone stream at both the entry zone and leading edge. Exaggerated loss of interneurons from migratory streams and dispersion throughout the cortical wall, including in the laterally positioned entry zone, were likely due to the extended duration of SP600125 treatment (24 hours of treatment in Figure 3.2 and 3.3 versus 12 hours of treatment in Figure 3.1). Following 24 hours of JNK inhibition, cortical interneurons accumulated in the cortical plate and the ventricular zone regions resulting in statistically significant changes in their 
radial distribution at the entry zone (two-way ANOVA: $F_{(18,120)}=2.579 ; 20 \mu \mathrm{M} \mathrm{p}=0.0012$; $F_{(18,120)}=3.292 ; 40 \mu \mathrm{M} p<0.0001$ ) and leading edge (two-way ANOVA: $F_{(18,120)}=2.785 ; 20 \mu \mathrm{M}$

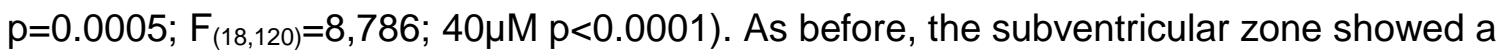
significant decrease in the abundance of cortical interneurons indicating that JNK inhibition led to evacuation of the SVZ/IZ stream (Figure 3.2J and 3.3J). Additionally, stream spreading and inconsistencies/gaps were often observed in the MZ, and rounded cortical interneurons without migratory processes were found in the cortical plate, especially at the leading edge (Figure 3.2F and 3.3F).

Washout slices showed evidence for recovery in some regions of the radial axis (Figure 3.2J, 3.3J). In slices that were washed out from treatment with $20 \mu \mathrm{M}$ SP600125, recovery of interneuron distribution was documented in the cortical plate and ventricular zone at the entry zone (Figure 3.2). At the leading edge of the same slices, recovery of cortical interneuron distribution was found in the lower subventricular zone stream and lower portion of the cortical plate, where the subplate is located (Figure 3.2). A similar result was seen in slices that were washed out from treatment with $40 \mu \mathrm{M}$ SP600125 (Figure 3.3B). These slices showed recovery in the cortical plate with trends toward recovery in the ventricular zone (Figure 3.3D), however, recovery following washout of $40 \mu \mathrm{M}$ SP600125 was less complete than recovery following washout of $20 \mu \mathrm{M}$ SP600125.

Our results indicate that restoration of JNK signaling can partially recover radial distribution of interneurons in the cortical wall and lead to the reinvestment of migratory streams. This suggests that acute JNK inhibition misroutes cortical interneurons, but their ability to respond to environmental cues located in the radial axis of the cortical wall remains intact, since interneurons are apparently able to reorient and reengage in a tangential migratory program when JNK signaling is restored. This ability to recover may be dose-dependent, however, as 
extreme displacement from migratory streams brought about by severe loss of JNK signaling appears largely irreversible.

Pharmacological loss of JNK signaling causes cortical interneurons to leave the subventricular zone stream and spend more time in the cortical plate

To determine the dynamic behavior of cortical interneurons in the absence of JNK signaling, we live-imaged E14.5 slice cultures from DIx-CIE+ embryos in control and SP600125 treated conditions. Cortical interneurons were tracked in the subventricular zone to assess the orientation of their migratory trajectory, distance traveled, and velocity.

Cortical interneurons in slices treated with $20 \mu \mathrm{M}$ SP600125 showed dramatic evacuation from the subventricular zone stream when compared to controls that were imaged over the same 12hour period (Figures 3.4A, B). Indeed, in SP600125 treated slices, only 30.56\% (44/144 interneurons) of the cortical interneurons remained in the SVZ stream, whereas $69.44 \%$ (100/144 interneurons) of the interneurons departed. When compared to control slices, where $70.14 \%$ (101/144 interneurons) of interneurons remained in the SVZ and only $29.86 \%(43 / 144$ interneurons) departed, highly statistically significant differences were found (T-test $p<0.0001$; Figure $3.4 \mathrm{C}$ top). When the migratory orientations of interneurons traveling in or departing from the SVZ stream were quantified (144 interneurons and 12 movies for control and inhibitor), we found that JNK inhibited cortical interneurons spent more time in diagonal (35.36\%; 2282/6468 frames) and radial (22\%; 1460/6486 frames) orientations and less time in tangential orientations (42.64\%; 2744/6486 frames) compared to controls, which spent 23.07\% (929/3985 frames) of their time traveling diagonally, 7.36\% (310/3985 frames) of the time traveling radially, and $69.56 \%$ (2746/3985 frames) of the time traveling tangentially. When compared, highly significant differences were found (T-test: Diagonal: $p=0.002$; Radial: $p<0.0001$; Tangential: $p<0.0001$; Figure 3.4C middle). Cortical interneuron velocity (T-test: Max, Mean Min, St. Dev: $p<0.0001$; Figure 3.4C bottom), displacement (T-test: $p<0.0001$, Figure 3.5A) and track straightness (T- 
test: $p<0.0001$, Figure 3.5B) were significantly decreased in the pharmacologically treated slices.

Cortical interneuron behavior in the cortical plate was also measured by tracking interneurons that entered a standardized box fit to the margins of the cortical plate. In SP600125-treated conditions, cortical interneurons that entered the cortical plate were less likely to have a tangential orientation, and more likely to have a diagonal orientation (Figure 3.4D, left). The length of time that interneurons spent in the cortical plate was statistically longer in SP600125treated slices compared to controls (T-test: $p<0.0001$; Figure 3.4D, middle), and accordingly, interneurons in SP600125-treated slices displayed decreased migratory velocity in the cortical plate region (Figure 3.4D, right).

Together, our results indicate that JNK signaling is required to maintain the tangential orientation and migratory velocity of cortical interneurons traveling in the SVZ stream. The robust infiltration of the cortical plate upon JNK inhibition suggests that downregulation of JNK signaling could mediate the timing of cortical interneuron departure from migratory streams and subsequent entry into the cortical plate.

Genetic loss of JNK signaling causes cortical interneuron stream dispersion and cortical plate accumulation

In order to determine if genetic loss of JNK function disrupts the integrity of migratory streams, ex-vivo slices from an allelic series of JNK mutants were analyzed. Since Jnk1;Jnk2 double mutants are early embryonic lethal (Kuan et al., 1999), and combinatorial loss of Jnk1 and Jnk2 has a greater effect on interneuron migration than loss of Jnk1 alone (Myers et al., 2014), we conditionally deleted Jnk1 from interneurons of Jnk2 null embryos, and then further examined the contribution of Jnk3 by conditionally deleting Jnk1 from interneurons of Jnk2 null;Jnk3 heterozygotes or Jnk2;Jnk3 double knockouts to completely eliminate JNK signaling in cortical interneurons. Organotypic slices from the allelic series of JNK mutant brains were prepared at 
E14.5 and imaged live for 18 hours. Cortical interneurons in both conditional triple mutant mice showed a shift in radial distribution from the subventricular zone to the cortical plate region (Figure 3.5). At first glance, the SVZ stream in the conditional triple mutant mice disperses and cortical interneurons accumulate within the cortical plate compared to the Jnk1; Jnk2 mutant controls.

In order to evaluate whether genetic removal of JNK function altered the distribution of interneurons in the cortical wall, still movie frames at $0,6,12$, and 18 hours from Jnk $1^{t / f / 1}$; Jnk $2^{-1}$;

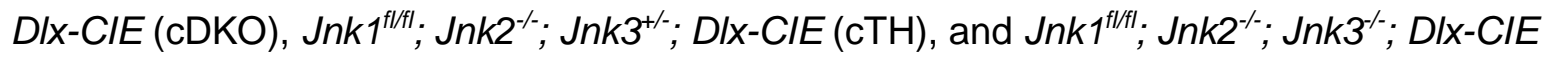
(cTKO) slices were quantified. At 0 hour, cortices from all four genotypes showed equivalent radial distribution of cortical interneurons (Figure 3.6, two-way ANOVA: $F_{(18,120)}=1.035$; $\mathrm{p}=0.4274$ ), suggesting that cortical interneurons are not significantly displaced in the $\mathrm{E} 14.5$ brain. By 6 hours, the distribution of cortical interneurons in the cortical wall begins to shift (Figure 3.6, two-way ANOVA: $\left.\mathrm{F}_{(18,120)}=2.383 \mathrm{p}=0.0028\right)$. Cortical interneurons in $\mathrm{cTH}$ and cTKO cortices show a statistically significant loss of cortical interneurons from the SVZ (Figure 3.6, Fisher's LSD test: Bin 5: $c D K O$ vs $c T H: ~ p=0.0069$; $c D K O$ vs $c T K O: p=0.019$; $B$ in 6: $c D K O$ vs cTH: $p=0.0131$; $c D K O$ vs $c T K O: p=0.0355$ ), statistically significant increases in the MZ (Figure 3.6, Fisher's LSD test: $B$ in $1: c D K O$ vs $c T H: p=0.0069$; $c$ DKO vs $c T K O: p=0.0029$ ), and trends toward significant increases in the upper cortical plate (Figure 3.6, Fisher's LSD test: Bin 2: cDKO vs cTH: $p=0.0627$; $c D K O$ vs $c T K O: p=0.0613$ ). At 12 hours, the $M Z$ stream and upper cortical plate region in the $\mathrm{CTH}$ and cTKO cortices display statistically significant increases cortical interneuron distribution while the SVZ stream is further compromised (Figure 3.6, twoway ANOVA: $F_{(18,120)}=4.037 ; p<0.0001$; Fisher's LSD test: Bin 6: $c D K O$ vs $c T H: p=0.0014$; cDKO vs cTKO: $p=0.0395$; $B$ in 7 : $c D K O$ vs $c$ TH: $p=0.0146$; $c D K O$ vs $c T K O: p=0.0004$; $B$ in 8 : cDKO vs cTKO: $p=0.0174)$. By 18 hours, the distribution of cortical interneurons has drastically changed throughout the radial axis. The SVZ stream counts reveal dramatic loss of cortical 
interneurons (Figure 3.6, two-way ANOVA: $F_{(18,120)}=4.069 ; p<0.0001$; Fisher's LSD test: Bin 6: cDKO vs cTH: $p=0.0356$; $B$ in 7 : $c$ DKO vs $c T H: ~ p=0.0156$; $c$ DKO vs $c T K O: p=0.003$; $B$ in 8 : cDKO vs $c T H: p=0.0055$; $c D K O$ vs $c T K O: p=0.014$ ), while cortical interneurons subsequently fill the MZ stream (Figure 3.5, Fisher's LSD test: Bin 1: cDKO vs cTH: $p=0.0054$ ), IZ (Figure 6, Fisher's LSD test: Bin 4: cDKO vs cTH: $p=0.0054$ ), and cortical plate region (Fisher's LSD test: Bin 2: $c D K O$ vs $c T H: p=0.0102$; $c D K O$ vs $c T K O: ~ p=0.0011$; $B i n$ 3: $c D K O$ vs $c T K O: p=0.0063$ ).

These results indicate that genetic loss of JNK signaling leads to dispersion of cortical interneurons from the SVZ stream and premature entry into the cortical plate. The incomplete evacuation of interneurons from the SVZ stream of conditional triple knockouts compared to the pharmacologically treated slices may suggest that complete loss of all JNK signaling increases interneuron dispersion through non-autonomous mechanisms.

Genetic loss of JNK signaling causes interneuron clustering in the cortical plate and intermediate zone regions

In order to assess cortical interneuron stream integrity in-vivo, we evaluated cortices from conditional double and triple knockout embryos at E15.5. Compared to cDKO cortices, the subplate stream was sparsely populated at more dorsal-medial regions of the cortex and almost unrecognizable laterally in cTH, and cTKO (Figure 3.7A-C). Moreover, clusters of cortical interneurons were found in the cortical plate and intermediate zone at the entry region of the cortex in $\mathrm{CTH}$, and cTKO (Figure 3.7B2, 3.7C1-3), which varied in size, number, and location within the cortical plate to intermediate zone region. The severity of interneuron clustering increased with genetic deletion of Jnk3: absent in cDKO cortices, sparse in cTH cortices, and prevalent in cTKO cortices. Cortical interneurons residing in clustered areas appeared nonmigratory as leading processes were small or absent in coronal sections (Figure 3.7B2, 3.7C13). Despite having rounded cell bodies and little to no migratory processes, interneurons within clusters did not label for cleaved caspase 3 , a marker of apoptotic cell death, or prematurely 
express markers of differentiation, including parvalbumin or calretinin (data not shown).

Disruptions to the MZ (open arrows, Figure 3.7), subplate (solid arrows, Figure 3.7), and SVZ stream (asterisks, Figure 3.7) were often present near clustered regions, indicating that interneurons likely evacuate streams adjacent to where clusters are forming (Figure 3.7B, C).

These data suggest that cortical interneuron clusters are associated with the loss of Jnk3 from Jnk1; Jnk2 conditional double knockout mice. This could be due to the evacuation of the MZ, subplate, and SVZ streams, which appear broken and devoid of interneurons in regions where clusters exist. The ablation of all JNK signaling from cortical interneurons may affect the polarity of the cortical interneurons after they disengage from migratory streams and enter the cortical plate, since they appear rounded in shape with no prominent leading processes, but show no sign of apoptotic cell death or post-migratory differentiation.

Cortical interneuron clusters form from the marginal zone stream in JNK deficient cortices

To determine the origins of cortical interneuron clusters, we further assessed our live imaging movies from E14.5 JNK deficient mutants to analyze the behavior of cortical interneurons that accumulated in the cortical plate. Cortical interneurons in cTKO cortices developed clusters formed by cells escaping the $M Z$ stream and invading the cortical plate during the first 6 hours of the movies (Figure 3.8). Interneurons from the subplate and SVZ region of the cTKO slices infiltrated the cortical plate, as well. These aberrant migratory behaviors were not observed in cDKO cortices (Figure 3.8). However, similar but less prominent defects were seen in movies obtained from the cTH cortices (data not shown), matching our in vivo findings. The accumulation of cortical interneurons within the cortical plate was quantified by counting the number of cortical interneurons (10 movies from 5 slices/genotype) within a 100X50 $\mu \mathrm{m}$ box. The cTKO cortices accumulated the most interneurons in the cortical plate at $0,3,6,9$, and 12 hours (Figure 3.9: two-way ANOVA: $\left.F_{(8,60)}=3.562 ; p=0.0019\right)$. 
Our data suggest that the loss of Jnk3 from Jnk1 and Jnk2 deficient interneurons promotes departure from migratory streams and entry into the cortical plate. The newly invading cortical interneurons then promptly recruit nearby interneurons from the subplate and SVZ migratory streams. These alterations in interneuron migration could be explained by an autonomous change, such as in the expression of cell surface guidance receptors or downstream signaling, or due to non-autonomous changes in environmental guidance cues that promote entry into the cortical plate and intermingling with other stream exiting interneurons.

\section{Cortical interneuron clusters originate in regions of radial glial disruption}

In order to determine if the cytoarchitecture of the developing cortical plate was disrupted at sites of interneuron clusters, we investigated the integrity of radial glial scaffolding in cDKO and cTKO embryos using Nestin labeling. Radial glial cells in the cortex of cTKO were found to be disrupted in areas of interneuron clusters compared to cDKO cortices (Figure 3.10). Radial glial scaffolding was not well organized in cTKO cortices (Figure 3.10). Endfoot detachment from the pial surface was seen in some, but not all embryos (Figure 3.10). Prevalence of radial glial scaffolding seemed sparse and the structure was disrupted with missing or spindly projections (Figure 3.10). In addition, Hoechst staining revealed potential disruptions to migrating excitatory neurons (Figure 3.10). Together, our data suggest that cortical interneuron clustering occurs where there are disruptions in radial glial scaffolding and/or potential disturbances in the cellular architecture of the cortical plate.

\section{Discussion}

In present study, we found that the tangential progression of cortical interneurons in migratory streams depends on the JNK signaling pathway. Pharmacological inhibition of JNK signaling using ex-vivo slice cultures revealed a dose-dependent dispersion of cortical interneurons from migratory streams. Furthermore, displaced interneurons were able to repopulate migratory 
streams upon removal of the pharmacological blockade, indicating that migratory stream dispersion is partially recoverable. Live imaging of ex-vivo slice cultures showed that acute JNK inhibition led to rapid stream dispersion, as JNK-inhibited cortical interneurons switched from tangential to radial modes of migration, exited the SVZ stream, and infiltrated the cortical plate region. Similar to acute pharmacological inhibition, deletion of all three Jnk genes from cortical interneurons resulted in dispersion from migratory streams in ex vivo slices. In vivo, we found that complete loss of JNK signaling from interneurons led to the accumulation of cortical interneurons in clusters within the cortical plate and intermediate zone region of the developing cortex. Live imaging revealed that clusters of cortical interneurons were formed from pockets of MZ-derived interneurons that dipped into the cortical plate and intermingled with other interneurons that evacuated from the SVZ stream. Additionally, the normal columnar architecture created by radial glial scaffolds was perturbed in regions of the cortical plate that were populated by clusters of cortical interneurons. Together, these results suggest that JNK signaling is required to maintain the integrity of cortical interneuron migratory streams and that JNK signaling levels may gate the entry of interneurons into the cortical plate.

\section{JNK signaling and cortical interneuron migration}

JNK signaling is known to direct numerous aspects of cortical development including the migration of radially and tangentially migrating neurons (Westerlund et al., 2011; Myers et al., 2014), and leading process branching (Gdalyahu et al., 2004), and axonogenesis (Oliva Jr et al., 2006). Additionally, mutations in JNK genes, specifically JNK3, are implicated in neurodevelopmental disorders including intellectual disability and epilepsy (Shoichet et al., 2006; Kunde et al., 2013), which might arise from the dysfunction of cortical interneurons. Our previously published work demonstrated that JNK signaling, specifically Jnk1, plays an important role during early cortical interneuron migration. Pharmacological blockade of JNK signaling delayed the initial entry of cortical interneurons into the cerebral cortex (Myers et al., 
2014). Furthermore, genetic ablation of Jnk1, or combinatorial deletion of Jnk1 and Jnk2 within cortical interneurons, also delayed their entry into the cortex (Myers et al., 2014). Additionally, we found that the orientation of cortical interneurons in migratory streams and their radial positioning in the cortical wall was disrupted, suggesting that JNK signaling might also be important for the initial assembly of cortical interneurons into organized streams (Myers et al., 2014). The current study is the first to suggest that JNK signaling plays a role in migratory stream maintenance and mediating the tangential to radial transition that interneurons make as they leave migratory streams in order to populate the nascent cortical plate. Indeed, both pharmacological inhibition and genetic ablation of JNK signaling causes interneurons to switch from tangential to radial migration, depart from streams, and accumulate in the cortical plate. Although it is currently unclear how JNK signaling regulates the tangential progression of cortical interneurons in migratory streams or how the withdraw of JNK activity facilitates the tangential to radial switch, the JNK signaling pathway may intersect with other molecular pathways known to facilitate this key transition of migratory behavior

Stromal derived factor-1 (SDF-1)/Cxcl12 is a chemokine that serves as a chemoattractant to cortical interneurons that express the Cxcr4 and Cxcr7 chemokine receptors (Liapi et al., 2008; López-Bendito et al., 2008; Wang et al., 2011). In the absence of SDF-1 or the Cxcr4 or 7 receptors, cortical interneurons disperse from migratory streams, leading to premature accumulation in the cortical plate during pharmacological inhibition and in-vivo genetic ablation (Sánchez-Alcañiz et al., 2011; Wang et al., 2011; Abe et al., 2014). Specifically, cortical interneurons in Cxcr4, Cxcr7, and SDF-1 null mice transition to a radial orientation and migrate from the MZ and SVZ streams to deposit in the cortical plate (López-Bendito et al., 2008; Sánchez-Alcañiz et al., 2011; Wang et al., 2011; Abe et al., 2014), a phenotype that is very reminiscent of JNK deficient cortical interneurons. Early deposition of interneurons in the cortical plate is also seen in a mouse model of 22q11.2 deletion syndrome-a developmental disorder 
linked to schizophrenia and autism—and these migratory anomalies are at least partially caused by diminished expression of Cxcr4 in cortical interneurons (Meechan et al., 2012). Although JNK and chemokine signaling have not yet been linked in cortical interneurons, Cxcr4 is known to activate JNK signaling in breast cancer cells to promote invasion (Serrati et al., 2008). It is tempting to speculate, therefore, that JNK signaling acts downstream of Cxcr4 activation to facilitate tangential progression of cortical interneurons in migratory streams.

Intracellular signaling events that initiate dynamic changes in the cytoskeleton of leading processes are not well understood, but their precise spatial-temporal control is critical for orienting cortical interneurons during their migration. Interestingly, doublecortin (Dcx), a microtubule binding protein known to regulate leading process branching and migration of cortical interneurons (Friocourt et al., 2007), is a downstream target of JNK signaling (Gdalyahu et al., 2004; Kawauchi et al., 2005; Jin et al., 2010). Recent studies suggest that SDF-1 can modulate the branching of the leading process through DCX (Lysko et al., 2014). Therefore, a potential link exists between SDF-1 and JNK signaling in migrating cortical interneurons. Previous research by Lysko and colleagues indicate a role for protein kinase $A$ (PKA) phosphorylation of DCX in the migration of cortical interneurons. They established that in the presence of high levels of SDF-1, PKA phosphorylation of DCX is low allowing DCX to bind and stabilize microtubules and decrease branching compared to controls which could subsequently maintain the tangential trajectory seen during their early migration in streams.

Downregulation/low expression of SDF-1 or CXCR4 increased PKA phosphorylation of DCX initiating branching (Lysko et al., 2014), which could potentially mediate the tangential to radial transition during cortical interneuron migration. Consequently, it is possible that during low PKA phosphorylation of DCX, JNK-phosphorylation of DCX could mediate the binding of DCX to microtubules when SDF-1 is high to maintain cortical interneuron migratory streams, and when 
SDF-1 and JNK activity is low, PKA phosphorylation of DCX initiates new branch formation to mediate the tangential to radial transition. However, this still remains to be determined.

The primary cilium, a structure within the leading process of cortical interneurons, further assists in guiding their trajectory (Baudoin et al., 2012; Higginbotham et al., 2012). The primary cilium, comprised of microtubules, is assembled by intraflagellar transport (IFT) and extended to determine the direction of travel by sensing guidance cues from the surrounding environment. It contains receptors for many different guidance pathways including Neuregulin-1, Slit, and Cxcr4, a known regulator of JNK signaling (Serrati et al., 2008; Baudoin et al., 2012; Higginbotham et al., 2012; Métin and Pedraza, 2014). Mutations in the ciliary protein, Arl13b or disruptions to sonic hedgehog $(\mathrm{SHH})$ pathway are known to disrupt the assembly of the primary cilium and the localization of the aforementioned receptors which in turn could affect their migration by influencing the downstream activation of JNK signaling (Baudoin et al., 2012; Higginbotham et al., 2012). As previously discussed, expression of Cxcr4 in the presence of SDF-1 distinctly promotes tangential travel of cortical interneurons and could do so through activation of JNK signaling and further DCX (Gdalyahu et al., 2004; Lysko et al., 2014). However, upregulation of other signaling pathways could initiate other roles for JNK in migration. Baudoin and colleagues (2012) found that expression of SHH promotes the tangential to radial transition in cortical interneurons through the assembly of the primary cilium. In the presence of $\mathrm{SHH}$, patched releases smoothened to move into the cilium along with anterograde transport of IFT proteins to further elongate the cilium (Baudoin et al., 2012). Kif motor proteins are involved in binding cargo for anterograde transport. JNK interacting proteins (JIP) bind cargo to Kifs indicating that JNK signaling may be involved in the anterograde movement of proteins to promote the growth of the primary cilium (Bowman et al., 2000; Verhey et al., 2001). Further, Baudoin and colleagues (2012) suggest that functional IFT could mediate interactions between cortical interneurons and cortical interneurons and other cells within the 
cortex. Therefore, having functional IFT could promote the expression of the adhesion molecules required for cortical interneurons to interact with other cell types, such as radial glia which might also require JNK signaling to help promote the tangential to radial transition (Petrich et al., 2002; Elias et al., 2010).

Another important regulator of the tangential to radial transition in cortical interneurons is the gap junction protein connexin43 (Petrich et al., 2002; Elias et al., 2010). Elias and colleagues (2010) found that overexpression of connexin43 promoted (and knockdown inhibited) the switch from tangential migration to radial migration in cortical interneurons by promoting adhesion between cortical interneurons and radial glial cells. Interestingly, studies in cardiomyocytes demonstrated that activation of JNK signaling can influence the expression of connexin43 (Petrich et al., 2002). Specifically, JNK activation was associated with downregulation of connexin43 (Petrich et al., 2002). Therefore, it is possible that low levels of JNK signaling in interneurons could regulate the timing of migratory stream exit by upregulating connexin43 expression, which would promote the tangential to radial transition.

\section{JNK and radial migration}

Our results suggest that in addition to migratory stream disruption, total genetic ablation of JNK from cortical interneurons causes disruptions to the columnar structure of radial glial cells and the disorganization of cells in the nascent cortical plate. In our triple knockout model, Jnk1 was conditionally deleted from the interneurons of Jnk2/Jnk3 double knockout mice. Therefore, all cells were deficient for Jnk2 and Jnk3, while only cortical interneurons (and other cells of the Dlx5/6 lineage) were deficient for all three Jnk genes. Previous studies have demonstrated unique roles for individual Jnk genes during radial migration into the developing cortical plate. Westerlund and colleagues (2011) showed that genetic ablation of Jnk1 enhances the migration of excitatory neurons into the cortical plate at E15.5, and disruptions to the subplate and layer VI at E17.5. More recently, Zhang and colleagues (2016) found that knockdown of Jnk2 decreased 
migration into the cortical plate, but knockdown of Jnk3 did not perturb radially migrating neurons. This suggests that loss of Jnk2 could disrupt the laminar position of radially migrating excitatory neurons, however this remains to be determined in-vivo. The consequences for combinatorial loss of two or more Jnk genes on cortical development have not been evaluated prior to our studies, but it is possible that deletion of Jnk2 and Jnk3 could alter the migration or differentiation of excitatory cortical neurons, even if Jnk1 remains intact. Therefore, disruptions in radial glial architecture and the disorganization of the nascent cortical plate seen in our triple knockout mice may be due to constitutive loss of Jnk2 and Jnk3. It is also possible that complete loss of Jnk in cortical interneurons non-autonomously disrupts radial glia and neurons of the cortical plate in our triple mutants. Conversely, signals from disrupted radial glia could cause interneurons to cluster in regions where radial glial endfeet have detached from the pial surface. Cortical interneurons interact with radial glial cells when migrating in the developing cortex, and when probed by radial glial endfeet in the marginal zone, some interneurons respond by turning towards the cortical plate (Yokota et al., 2007). Moreover, disruption to radial glia perturbs interneuron migration in ferrets (Poluch and Juliano, 2007), again suggesting that cell-cell interactions between interneurons and radial glia are essential for normal aspects of cortical development. Molecular mechanisms underlying communication between cortical interneurons, radial glia, and potentially, radially migrating projection neurons, remain unknown, but it is possible that JNK signaling coordinates important features of this intercellular communication. Considerable work remains in order to untangle autonomous from nonautonomous functions of JNK during cortical development, as well as uncovering the unique roles for each of the Jnk genes in the process.

JNK3

Expression of the Jnk3 gene is localized to namely the central nervous system and therefore, may play a large role in neuronal migration and development (Kuan et al., 1999). Mutations in 
the JNK3 gene in humans have resulted in neurological impairment and disease (Shoichet et al., 2006; Kunde et al., 2013), however knockdown of Jnk3 in radially migrating neurons did not reveal a migration phenotype (Zhang et al., 2016). Therefore, investigating the role Jnk3 plays during cortical interneuron migration and development is pertinent. Our studies indicate a genetic dosage requirement for JNK signaling in cortical interneurons. Loss of Jnk3 in combination with loss of Jnk1 and 2 leads to departure of interneurons from migratory streams and clustering of interneurons in the cortical plate, as well as defects in the columnar structure of the radial glial cells and radially migrating projection neurons.

\section{Conclusions}

It remains clear that JNK signaling is intricately involved in the early migration of both cortical interneurons and radially migrating projection neurons. However, the precise temporal and spatial activity of JNK, as well as the autonomous versus non-autonomous functions of JNK, remains unknown. Therefore, elucidating upstream activators and downstream effectors of JNK signaling in cortical interneurons will be essential to determine intrinsic functions of JNK in cortical interneuron development. Indeed, deciphering mechanisms of JNK function is imperative for better understanding and ultimately treating neurodevelopmental and psychiatric disorders that impinge upon cortical circuit function. 
Figures
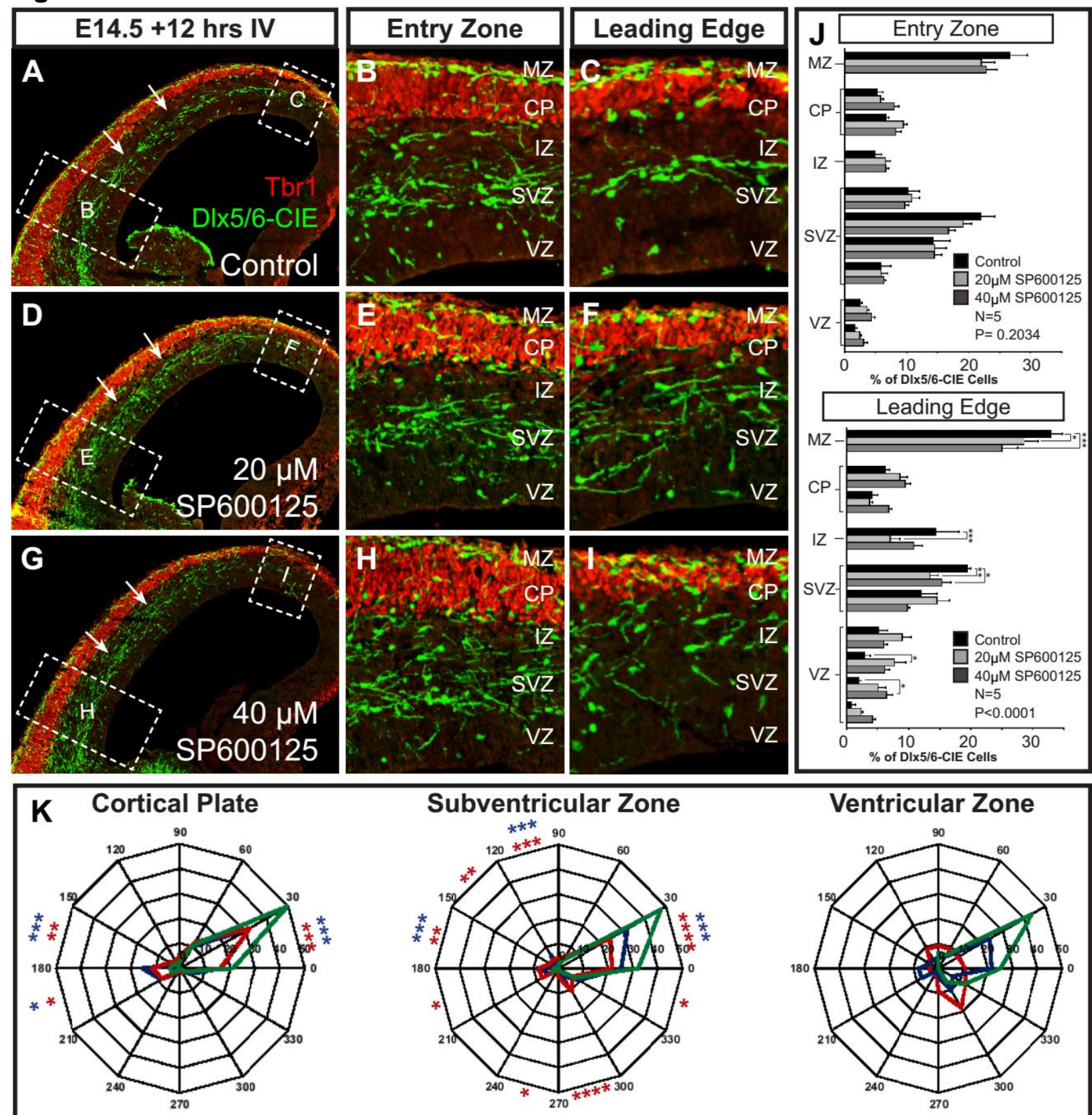

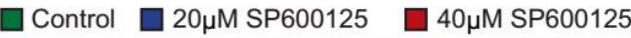

Figure 3.1: JNK-inhibition disrupts migratory stream integrity. DIx5/6-CIE+ cortical interneurons travel tangentially in migratory streams under control (A-C) conditions. SP600125-treatment leads to dose-dependent dispersion from both SVZ and MZ streams at $20 \mu \mathrm{M}(\mathrm{D}-\mathrm{F})$ and $40 \mu \mathrm{M}$ (G-I). Intermediate zone (arrows) fills with Dlx5/6-ClE+ interneurons (compare $\mathbf{D}, \mathbf{G}$ to $\mathbf{A} ; \mathbf{J}$ ) and interneuron orientation shifts from tangential $(B, C)$ to diagonal or radial $(E-F, H-I)$ after JNK 
inhibition. Differences between bins were determined post hoc by Fisher's LSD tests $\left({ }^{* * *} p<0.0001,{ }^{* * *} p<0.001,{ }^{* *} p<0.01,{ }^{*} p<0.05\right)$. JNK inhibition leads to a shift in migratory trajectories as measured by angular divergence from the tangential plane in the cortical plate/intermediate zone region, but not in the subventricular zone. Differences were determined by Chi squared analysis: cortical plate (150-180 degrees, $20 \mu \mathrm{M}: \mathrm{p}=0.0003 ; 40 \mu \mathrm{M}$ : $\mathrm{p}=0.0052$; 180-210 degrees, $20 \mu \mathrm{M}: \mathrm{p}=0.031 ; 40 \mu \mathrm{M}$ : $\mathrm{p}=0.0483)$; subventricular zone (90-120 degrees; 20 $\mu \mathrm{M}: \mathrm{p}=0.0001 ; 40 \mu \mathrm{M}: \mathrm{p}=0.0045,120-150$ degrees, $40 \mu \mathrm{M}: \mathrm{p}=0.0088 ; 150-180$ degrees, $20 \mu \mathrm{M}$ : $\mathrm{p}=0.0006 ; 40 \mu \mathrm{M}: \mathrm{p}=0.0026 ; 180-210$ degrees, $40 \mu \mathrm{M}: \mathrm{p}=0.0419$ and 240-270 degrees, $20 \mu \mathrm{M}$ : $\mathrm{p}=0.0598 ; 40 \mu \mathrm{M}: \mathrm{p}=0.0028 ; 270-300$ degrees; $20 \mu \mathrm{M}: \mathrm{p}=0.0863 ; 40 \mu \mathrm{M}: \mathrm{p}<0.0001$; and 330360 degrees, $40 \mu \mathrm{M}: \mathrm{p}=0.0109)(\mathrm{K})$ 

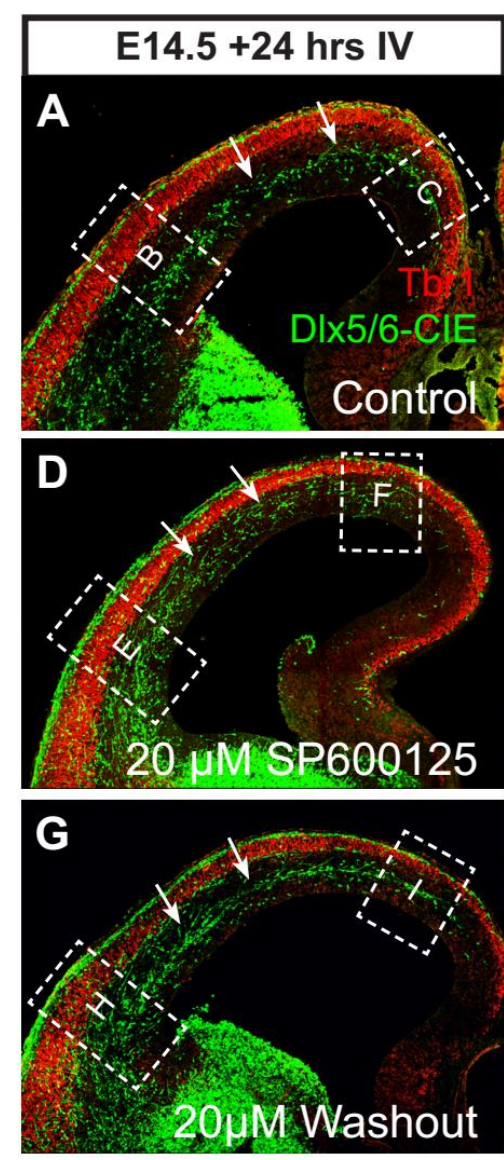
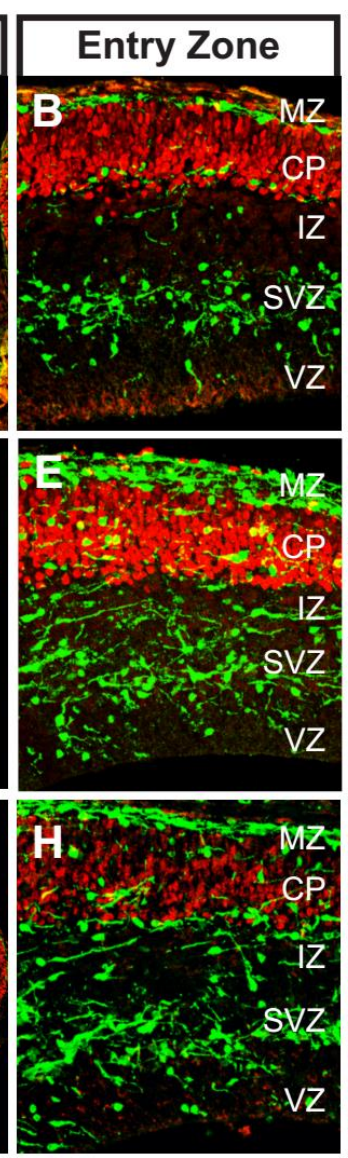
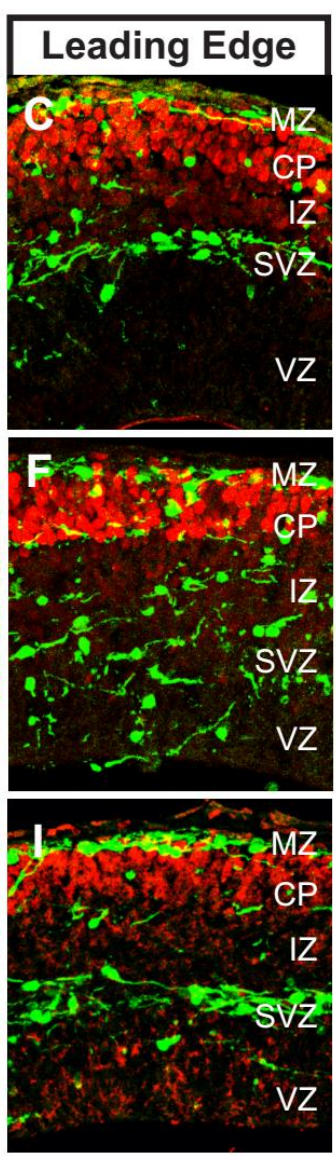

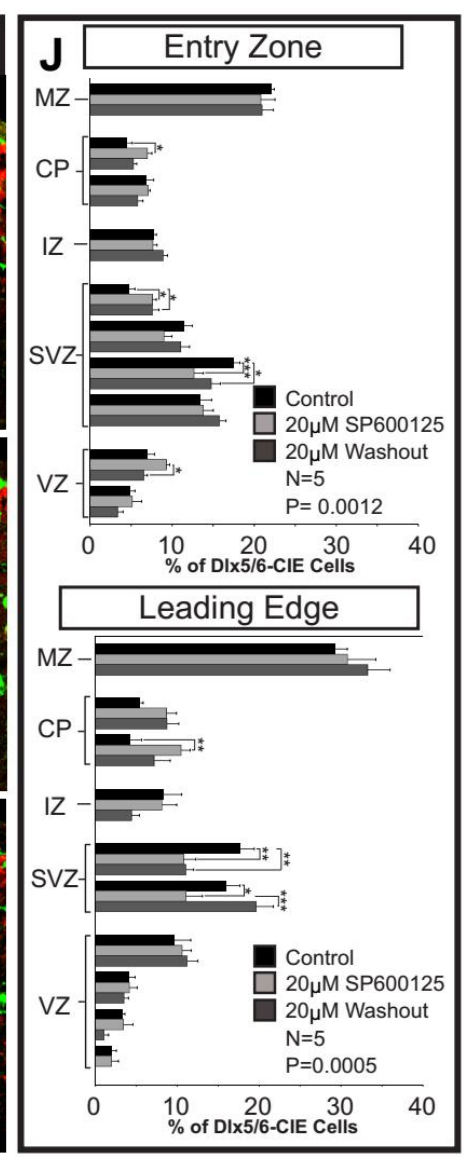

Figure 3.2: Removal of the JNK inhibitor improves migratory streams integrity. Cortical interneurons disperse from migratory streams during 24 hours of $20 \mu \mathrm{M}$ SP600125 (D-F) compared to controls (A-C). Washout of the SP600125 at 12 hours promotes recovery in the subventricular zone stream (G-I, J) after initial disruption. In addition, the cortical plate and intermediate zone regions show decreased accumulation (J) Differences between bins were determined post hoc by Fisher's LSD tests $\left({ }^{* * *} p<0.0001,{ }^{* * *} p<0.001,{ }^{* *} p<0.01,{ }^{*} p<0.05\right)$. 

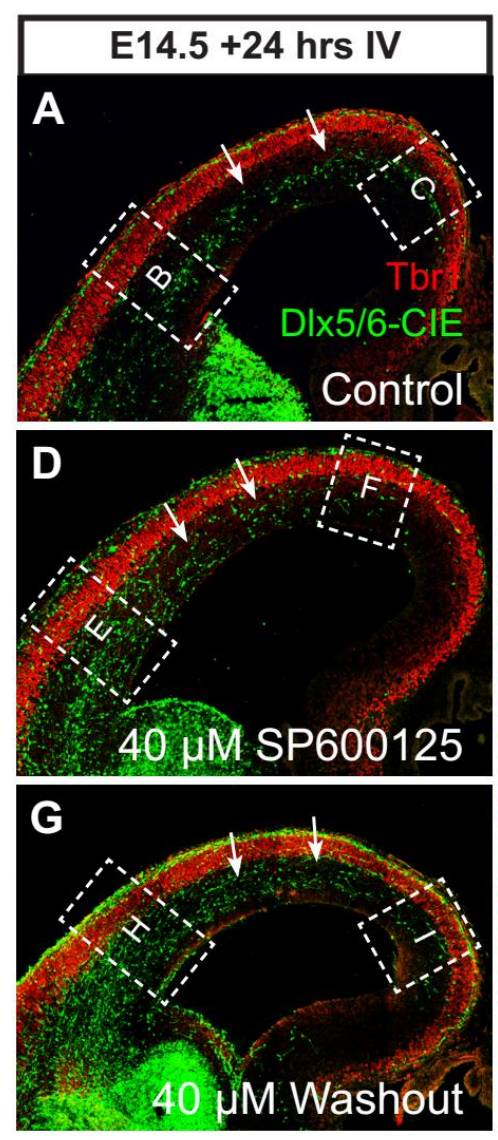
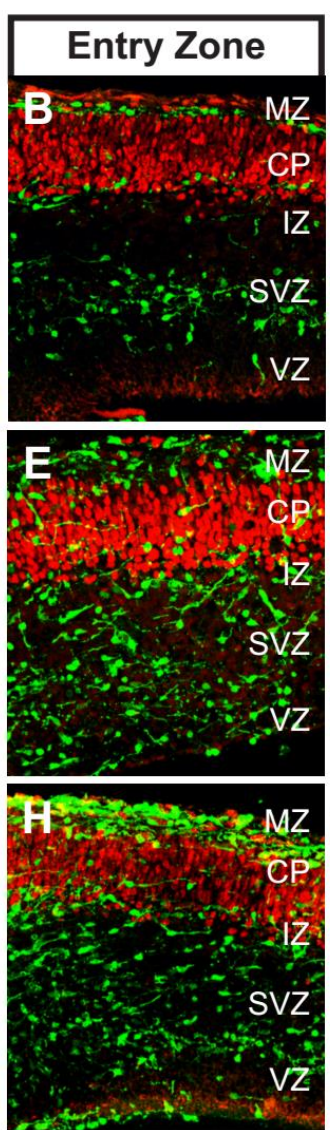
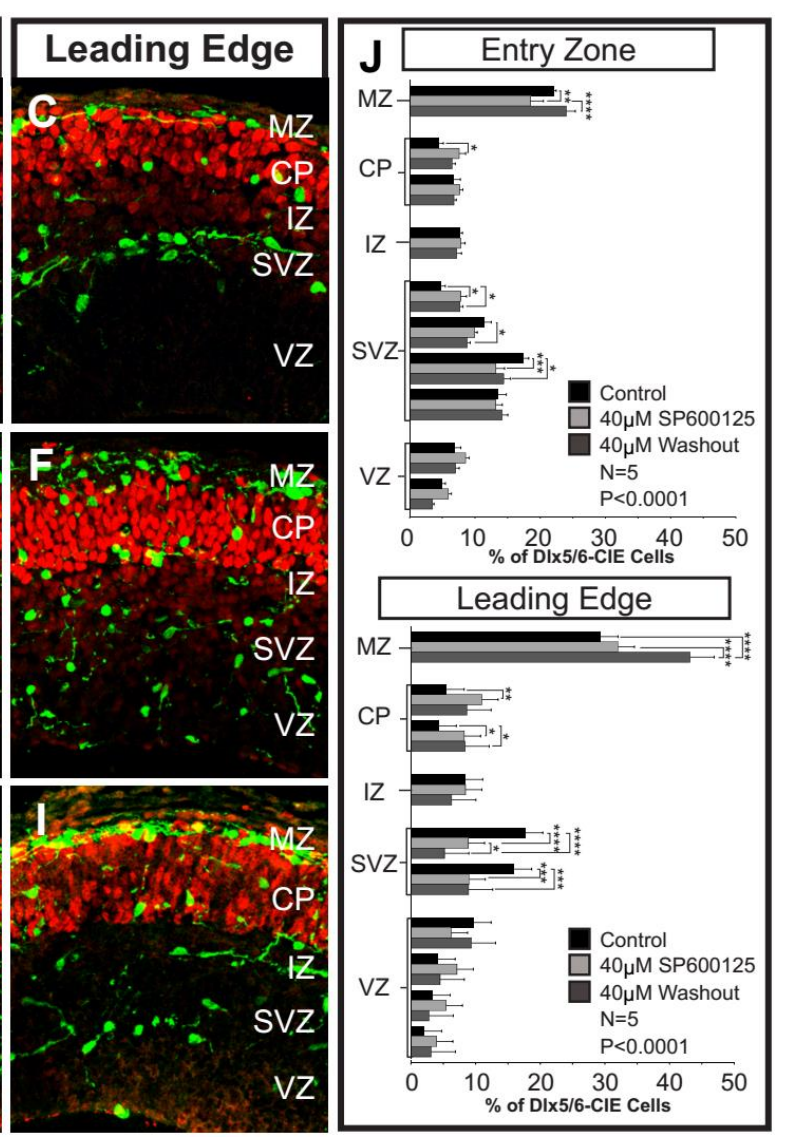

Figure 3.3: Cortical interneuron streams show trends of recovery in the ventricular zone (VZ) after JNK inhibition at $40 \mu \mathrm{M}$ concentration. Cortical interneurons disperse from migratory streams during 24 hours of $40 \mu \mathrm{M}$ SP600125 (D-F) compared to controls (A-C). Washout of the SP600125 at 12 hours promotes recovery in the VZ region (G-I, J) after initial disruption.

Differences between bins were determined post hoc by Fisher's LSD tests $\left({ }^{* * * *} p<0.0001\right.$, $\left.{ }^{* * *} p<0.001,{ }^{* *} p<0.01,{ }^{*} p<0.05\right)$. 

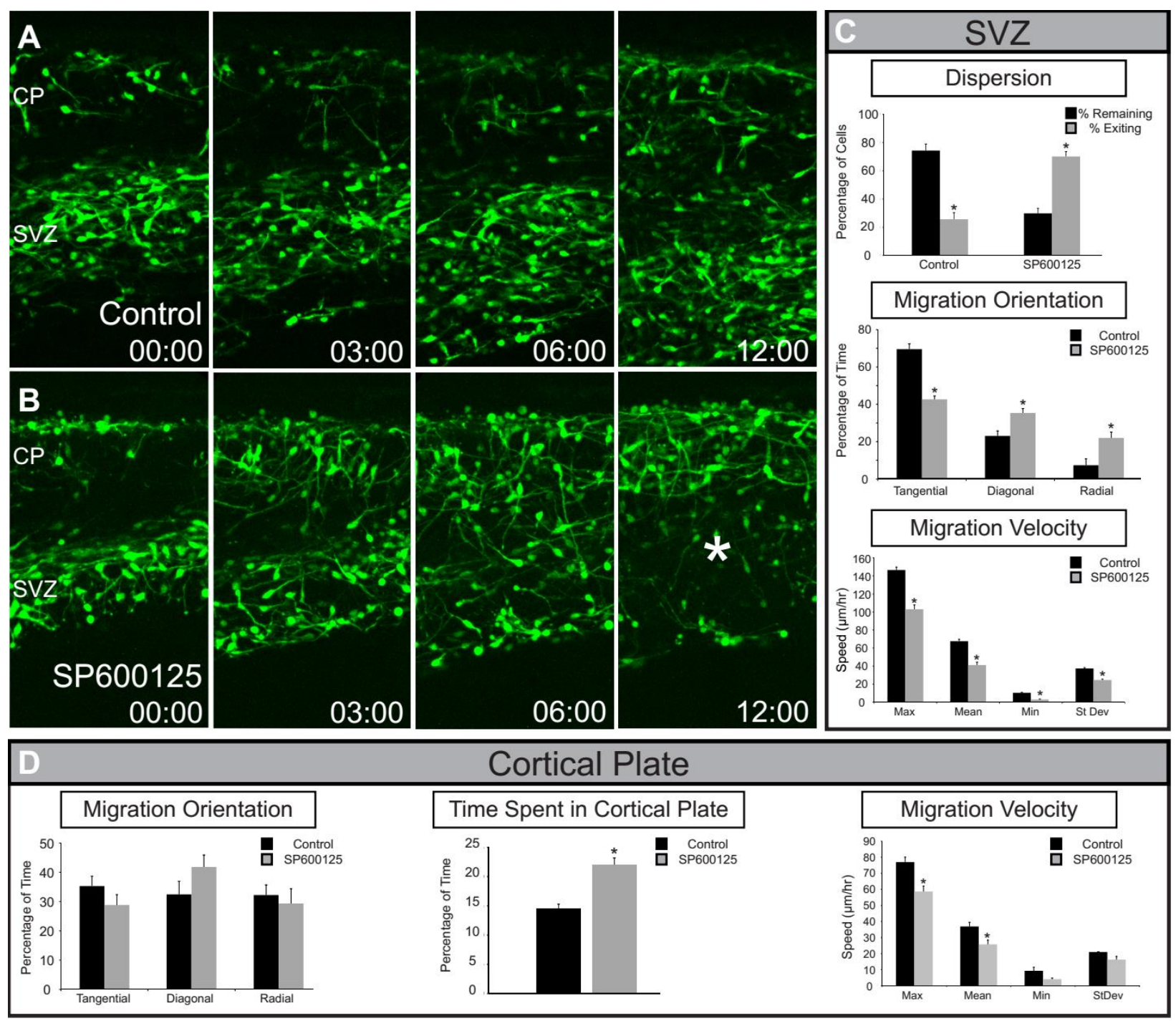

Figure 3.4: Tangential progression in migratory streams requires JNK activity. A. Dlx5/6-ClE+ interneurons travel in tangentially oriented migratory streams in control slices. B. Interneurons disperse from streams and vacate the SVZ within 12 hours of $20 \mu \mathrm{M}$ SP600125 exposure. C. SVZ-positioned interneurons in SP600125-treated slices travel slower and in a diagonal or radial trajectory compared to controls. D. Cortical interneurons spend more time and exhibit slower migration speeds in the cortical plate of JNK inhibited slices. 

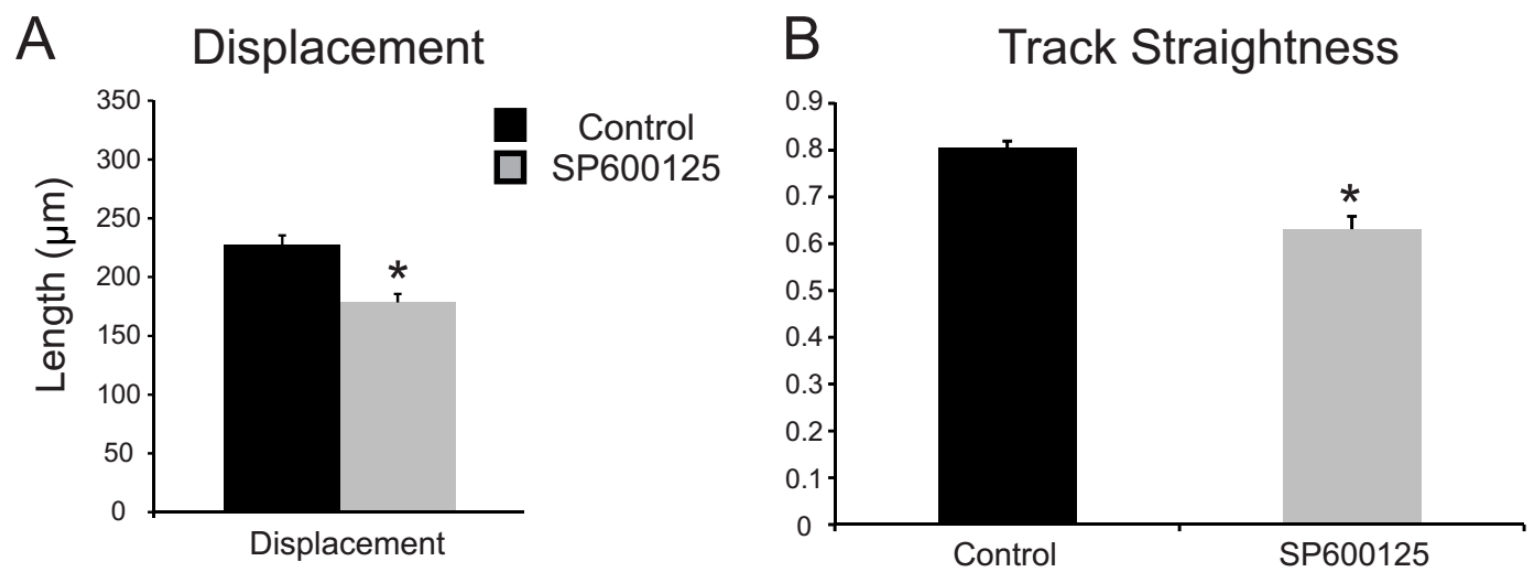

Figure 3.5: Cortical interneurons migrating within the SVZ of pharmacologically treated slices have decreased displacement and less straight tracks when compared to controls. 

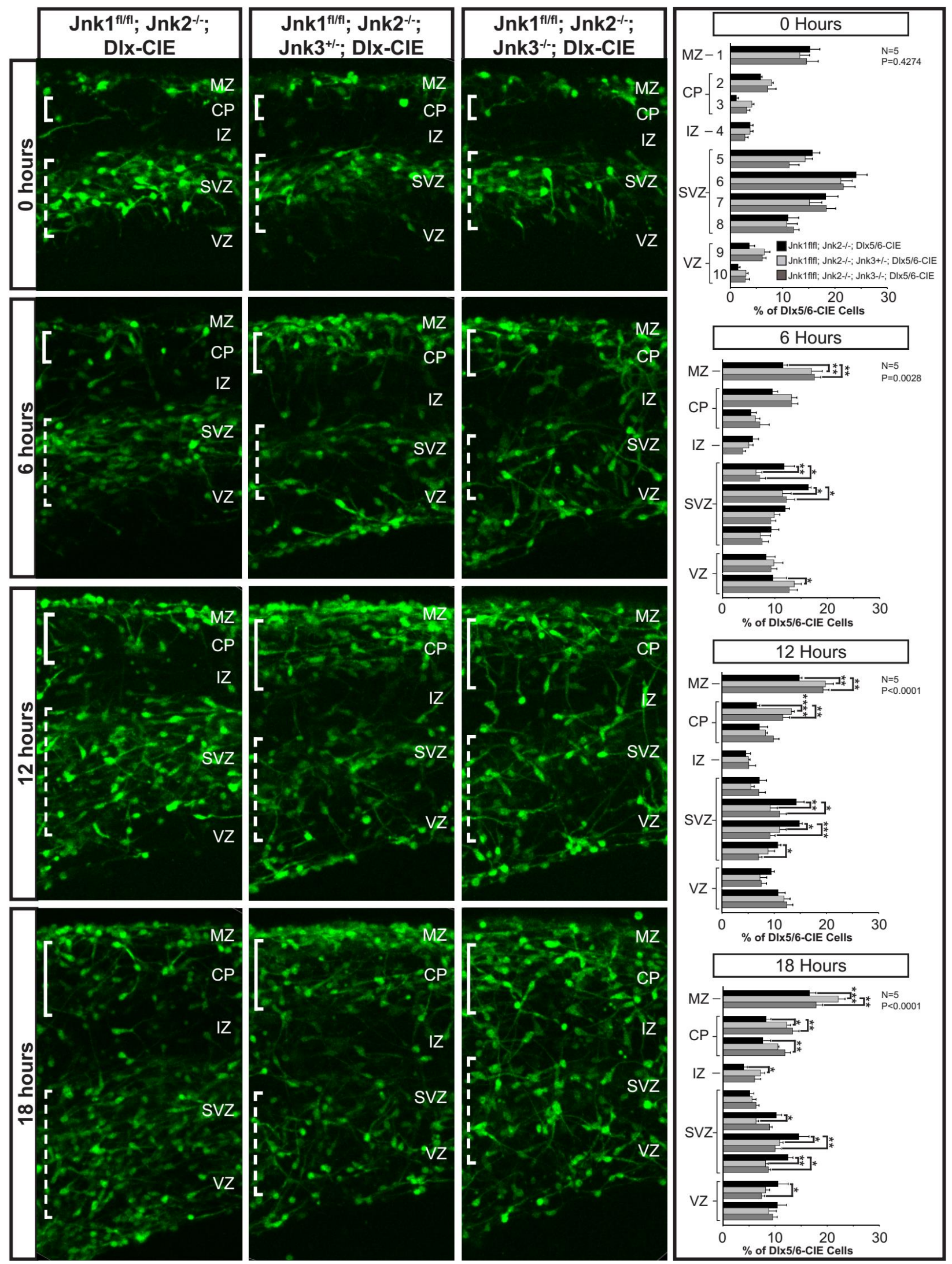
Figure 3.6: Genetic loss of JNK signaling from cortical interneurons disrupts migratory stream integrity. Cortical interneurons leave migratory streams during a period of 18 hours of ex vivo slice culture from embryonic day 14.5 in conditional triple knockouts compared to conditional double knockouts. Complete genetic loss of Jnk from interneurons leads to dispersion of cortical interneurons from the subventricular zone stream and accumulation in the cortical plate.

Differences between bins were determined post hoc by Fisher's LSD tests $\left(^{* * \star *} p<0.0001\right.$, $\left.{ }^{* * *} p<0.001,{ }^{* *} p<0.01,{ }^{*} p<0.05\right)$. 

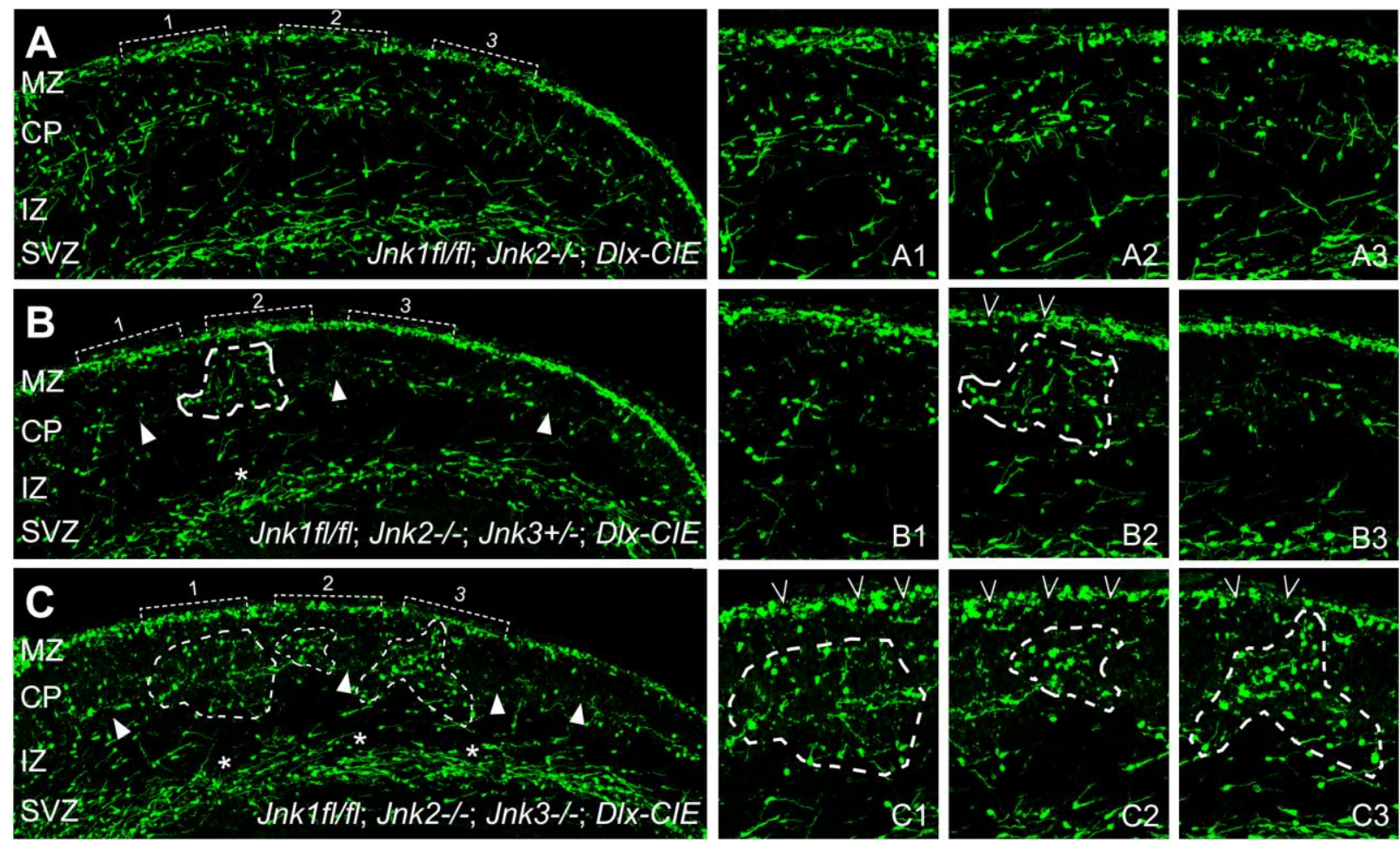

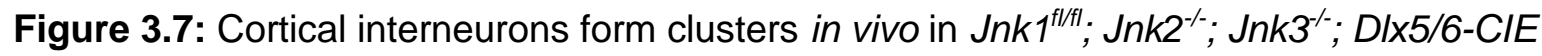
cortices at E15.5. Increasing allelic loss of Jnk3 in addition to loss of Jnk1 and Jnk2 causes cortical interneurons to cluster in the cortical plate and intermediate zone streams (A, B, C,

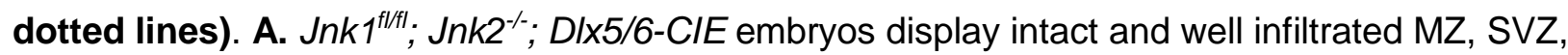
and subplate streams. B, C. Clustered patches of cortical interneurons disrupt the MZ (B2, C13, open arrows) subplate stream (B, C, solid arrows) and the SVZ (B, C; Astericks) in the

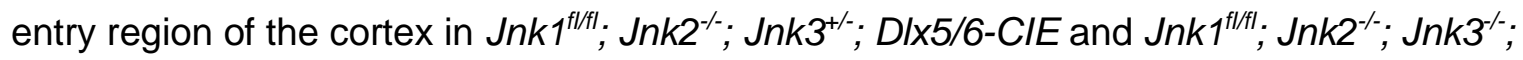
DIx5/6-CIE. Patches include rounded interneurons that appear non-migratory (B2, C1-3). 

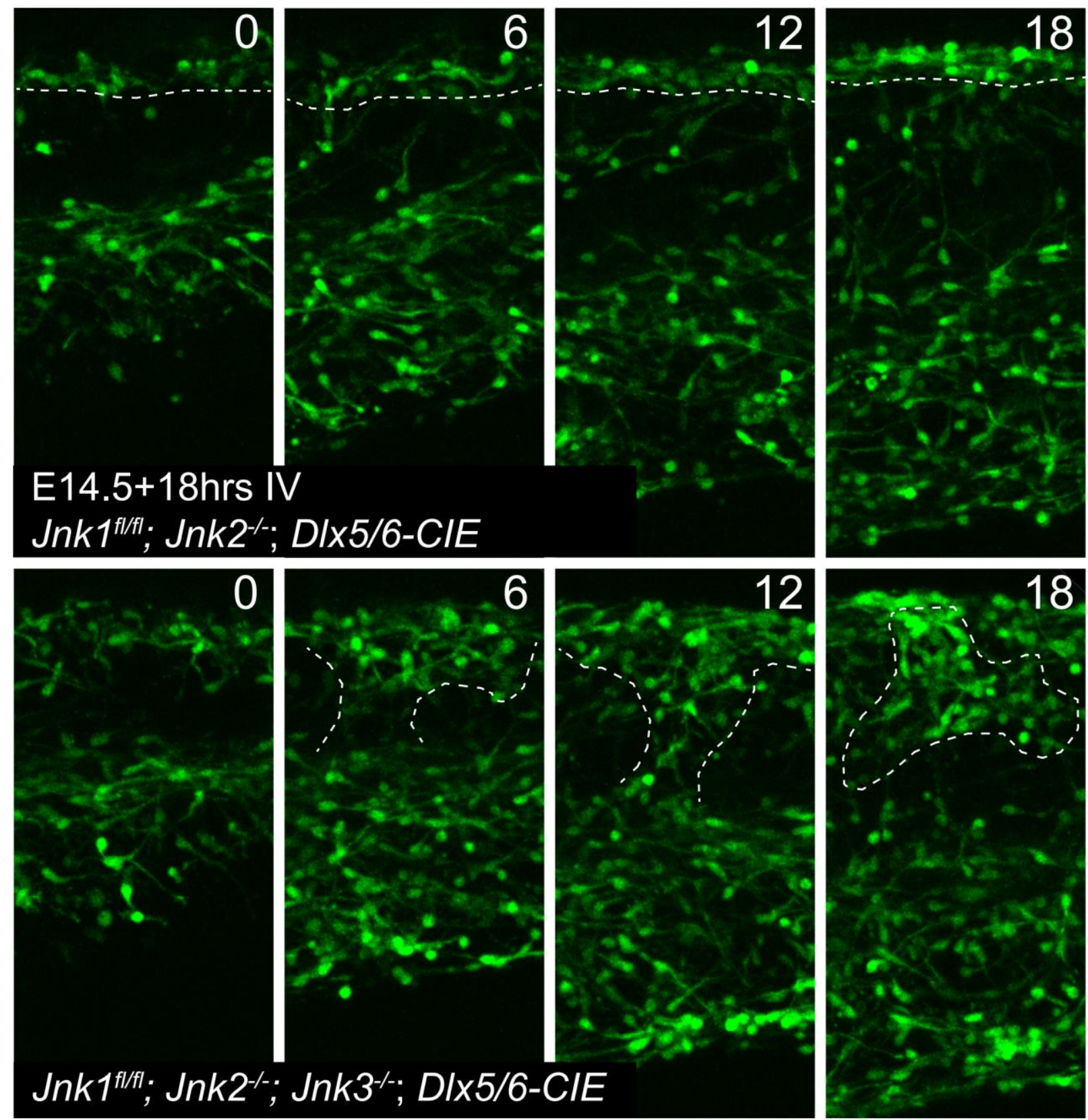

Figure 3.8: Cortical interneurons form clusters originating from the marginal zone stream. Top

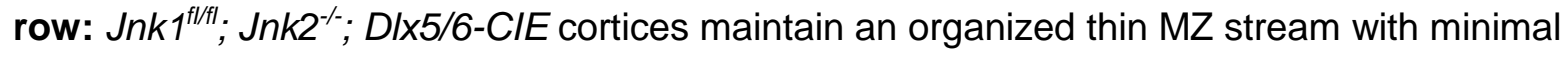
accumulation of cortical interneurons within the cortical plate. Bottom row: Jnk $1^{f l / f l}$; Jnk $2^{---}$; Jnk3 ^; DIX5/6-CIE cortices accumulate patches of cortical interneurons in the MZ stream that continue to protrude down into the cortical plate as time elapses and recruit cortical interneurons migrating within the SVZ stream 


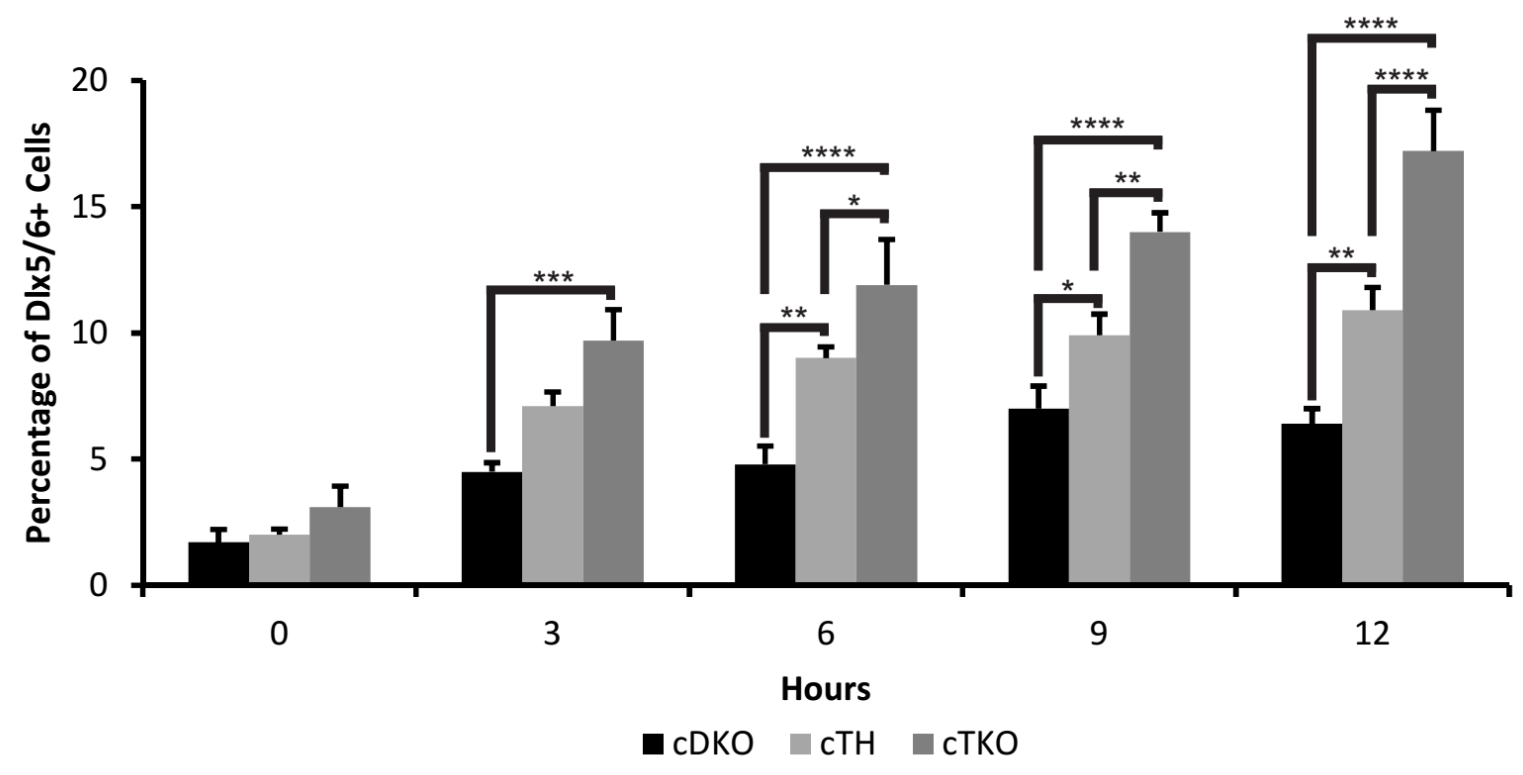

Figure 3.9: Cortical interneurons in cTKO cortices accumulated faster and more abundantly than cortical interneurons in cTH and cDKO cortices over 12 hours. Significance levels after performing two-way ANOVA are denoted as follows: ${ }^{* \star * *} p<0.0001,{ }^{* * *} p<0.001,{ }^{* *} p<0.01$, ${ }^{*} \mathrm{p}<0.05$ 

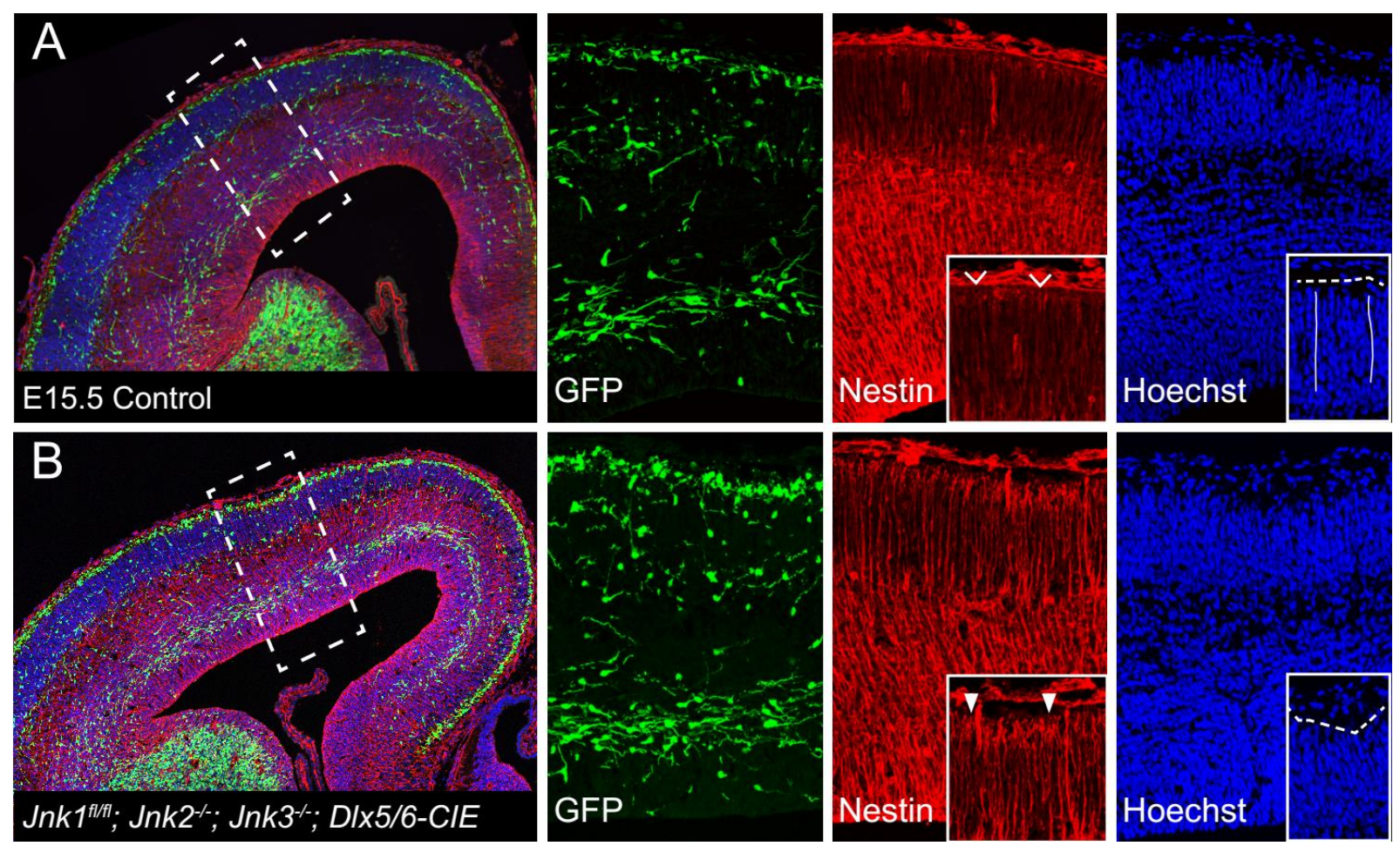

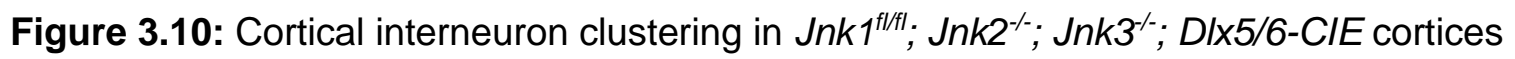

coincides with disruption to radial glia. Nestin labeling (red) reveals pial surface detachment of radial glial endfeet and incomplete columnar structure (B, inset, solid arrows) in Jnk $1^{f / f f l}$; Jnk2

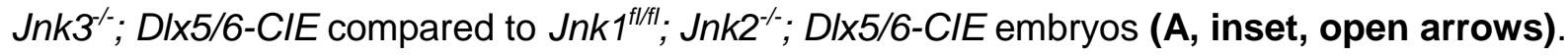
Neurons labeled with Hoechst in Jnk $1^{f / f t} ; J n k 2^{--} ;$DIx5/6-CIE are arranged in even columns (A, inset, white solid lines) unlike Jnk $1^{f l / f l} ;$ Jnk $2^{-/} ; J n k 3^{-/} ; D I \times 5 / 6-C I E$ which have lost their organization (B, inset). 
Chapter 4: Summary and Future Directions 


\section{Summary}

This work revealed a new and vital role for JNK signaling during early cortical interneuron migration. In chapter 2, we tested whether cortical interneurons migrating into the cortical rudiment required JNK signaling to enter and navigate the developing cerebral cortex. We used ex vivo slice pharmacology and in vivo genetic deletion to demonstrate that the loss of JNK signaling delayed the migration of cortical interneurons into the nascent cortical rudiment between E12.5 and E13.5. We established that this early delay is primarily due to elimination of Jnk1. Constitutive knockouts of Jnk1 showed delayed migration of cortical interneurons in caudal sections of the embryonic cortex, and the loss of one allele of Jnk2 in combination with Jnk1 delayed interneuron migration in both rostral and caudal sections. Elimination of only Jnk2 did not disrupt cortical interneuron migration, and loss of one allele of Jnk1 in combination with deletion of Jnk2 did not delay migration. Since dual constitutive deletion of Jnk1 and Jnk2 is embryonic lethal, we conditionally deleted Jnk1 in combination with constitutive deletion of Jnk2 using a DIx5/6-CRE-IRES-EGFP (DIx5/6-CIE) transgene to delete both Jnk1 and Jnk2 from cortical interneurons. Conditional double knockout (Jnk $\left.1^{f / f l} ; J n k 2^{-/} ; D / x 5 / 6-C I E\right)$ cortices had delayed migration compared to controls (Jnk $\left.1^{f / /+} ; J n k 2^{-/} ; D I \times 5 / 6-C I E\right)$. However, this delay was not determined to be solely autonomous, since in the absence of cortical guidance cues, JNKdeficient cortical interneurons migrated comparably to controls. Additionally, we found cortical interneuron streams to be disrupted. Cortical interneurons were not oriented tangentially within the SVZ stream and both the MZ and SVZ stream lacked integrity.

In chapter 3, we tested if JNK signaling was involved in the maintenance of cortical interneuron migratory streams. This study was completed between E14.5 and E15.5 when cortical interneuron streams are well-formed. Pharmacological blockade of JNK signaling in ex-vivo slice cultures at E14.5 showed evacuation of the SVZ stream with successive filling of the cortical plate, which was partially reversible when JNK inhibition was removed. Dynamic 
evaluation of cortical interneurons through time-lapse imaging showed that JNK-inhibited cortical interneurons transitioned from tangential migration to diagonal or radial migration and exited the SVZ stream at a higher frequency than controls. Further, when JNK-inhibited cortical interneurons reached the cortical plate they spent more time there than control interneurons. Next we sought to investigate the consequences of in-vivo loss of JNK signaling on cortical interneuron stream spreading and found no differences between cDKO's and $J n k 1^{t / /+} ; J n k 2^{--}$; DIx5/6-CIE control cortices in E14.5 + 18 hours of growth slices. However, deletion of Jnk3 in combination with Jnk1 and Jnk2 from cortical interneurons showed SVZ disruption and filling of the cortical plate that was similar to the SVZ dispersion and cortical plate filling seen in ex vivo slices during pharmacological inhibition using a pan-JNK inhibitor. In fact, the radial distribution of cortical interneurons lacking all three Jnk genes was almost identical to the distribution seen during pan-JNK inhibition. In-vivo analysis of E15.5 cortices lacking Jnk3 in combination with Jnk1 and Jnk2 identified clusters of cortical interneurons within the cortical plate in the entry region of the cortex with subsequent disruption to the subplate stream. Further, exploration of cortical plate filling in cTKO cortices revealed disruption and aggregation of cortical interneurons within the MZ stream. Live imaging of ex vivo slices revealed that cortical interneurons from the $M Z$ stream invaded the cortical plate and aggregated into clusters with cortical interneurons from the SVZ stream. This prompted us to look at the underlying architecture of the radial glia and the laminar allocation of projection neurons in the early cortical plate. Radial glial cells were disrupted along their radial axis. Decreased nestin labeling indicated loss of radial glial processes and disruptions to endfeet at the pial surface. The columnar organization of cells within the nascent cortical plate was also disrupted when compared to cDKO cortices.

This body of work suggests that JNK signaling plays an important role during the migration of cortical interneurons as they first enter the cerebral cortex and progress medially in streams of migratory cells. JNK mediates the integrity of migratory streams within the developing cortex, 
potentially by orienting cortical interneurons. However, it still remains unknown which JNK genes are responsible for this to occur and how these genes work in conjunction with each other to produce progressive movement in streams.

We know from this work that Jnk1 plays a dominant role in the progress of streams early on in migration, but this dominant role may change as the advancement of cortical interneuron streams in cDKO cortices catch up to $J n k 1^{f / /} ;$ Jnk2 $2^{-/}$; DIX5/6-CIE controls by E15.5 (Appendix Figure A1). At E13.5, cortical interneurons in cDKO cortices showed delays in migration and disruption to their radial distribution whereas $J n k 1^{f l /+}$; Jnk2 $2^{-/}$; DIX5/6-CIE cortices did not show this delay or disruption. Therefore, at early time points, one copy of Jnk1 is sufficient for normal migration. Two days later, at E15.5, the distribution and advancement of cortical interneurons is similar between $\mathrm{cDKO}$ and $J n k 1^{f / /+} ; J n k 2^{-/-}$; DIX5/6-CIE, which may indicate that the role of Jnk1 in cortical interneuron migration might change over time. It is unclear if Jnk1 could still play a role in the radial distribution of cortical interneurons within the developing cerebral cortex and if this influences the overall distribution of cortical interneurons in the mature cortex. The distribution of cortical interneurons in cDKOs and $J n k 1^{f /+}$; Jnk2 $2^{-/-}$DIx5/6-CIE cortices remain similar into adulthood at postnatal day 21 (Appendix Figure A2). However, it is still not known if the laminar position of cortical interneurons in either the adult cDKOs and Jnk $1^{f / /+}$; Jnk2 ${ }^{-/}$; DIx5/6-CIE cortex differs from a wildtype control brain. If the layer distribution of cortical interneurons is disrupted, Jnk1 and/or Jnk2 may become increasingly important during migration. Loss of just one allele of Jnk1 could be detrimental to interneuron migration and further laminar position within the cerebral cortex. If cortical interneurons are distributed normally in the adult cDKO, this could mean a switch in roles for these Jnk genes. Further, it is still unclear how and if Jnk3 is involved in cortical interneuron migration and if it could be compensating for Jnk1 or Jnk2 at these timepoints. 
To elucidate the role of Jnk3 in interneuron migration, and to untangle the differential contributions of each of the Jnk genes, we began a series of studies on mice with Jnk3 deletions. Preliminary results from constitutive genetic deletion of Jnk3 at E13.5 show no differences between the advancement of cortical interneuron migratory streams in $\mathrm{Jnk} 3^{+/}$and $J n k 3^{--}$. Therefore, Jnk3 may not play a significant role in the early entry of cortical interneurons into the cerebral cortex when deleted independently of other Jnk genes (Appendix Figure A3). However, by E15.5 loss of Jnk3 in combination with loss of Jnk1 and Jnk2 creates clustering of cortical interneurons and has detrimental effects on radial glia and the structure of the developing cortical plate. Additionally, we do not know if Jnk3 compensates for the loss of Jnk1 or the combinatorial loss of Jnk1 and Jnk2 at E13.5 or E15.5. Pharmacological blockade of JNK signaling at E12.5 prevented a large majority of cortical interneurons from entering the cerebral cortex. Therefore, the delayed entry and loose organization of cortical interneuron streams that is found in Jnk1 and Jnk2 deficient cortices could be due to compensation by Jnk3.

\section{Future directions}

While the current work breaks down the involvement of Jnk1 and Jnk2 during early migration and the additive effect of loss of Jnk3 to Jnk1 and Jnk2 mutants in migratory stream integrity, further work is needed to elucidate the involvement of each individual Jnk gene throughout cortical development. It is clear from our work that the role of Jnk1 and Jnk2 changes throughout cortical interneuron migration, specifically between E13.5, when interneurons first enter the cerebral cortex, and E15.5, when the leading edge of cortical interneuron migration reaches the medial most aspect of the cortical rudiment (Chapter 2, Appendix Figure A1). Therefore, future experiments should focus on examining the cell type and developmental expression of each Jnk gene. This will help us further understand the role of each gene during the course of both tangential migration of cortical interneurons and radial migration of excitatory neurons during cortical development. 


\section{Experiments:}

- In order to evaluate the expression of Jnk transcripts in cortical interneurons at different age points, flow sorting and/ or in-situ hybridization could be utilized.

- A time course using cell sorted cortical interneurons and respective cortices to investigate the expression levels of Jnk genes within cortical interneurons and the surround cortex to further understand the temporal changes of each Jnk gene during the cortical development using qRt-PCR.

- In-situ hybridization could be utilized to visualize the expression of each Jnk gene in single, double, and triple Jnk mutants. This method would help us to understand where each Jnk is expressed during each time point and which Jnk genes could be compensating for others in Jnk mutants.

Further, it still remains unclear how JNK signaling affects the temporal distribution of neurons within the cortex. Previous research has focused on the importance of JNK signaling in radial migration and development. Westerlund and colleagues (2011) demonstrated that constitutive loss of Jnk1 enhances the migration of excitatory neurons into the cortical plate. Contrary to the function of Jnk1, Zhang and colleagues (2016) found that knockdown of Jnk2 in migrating excitatory neurons slows migration, however found no effect was found when Jnk3 was depleted. This effect may be due to where the Jnks are localized and function within the excitatory neurons. Previous research by Westerlund and colleagues showed that the localization of Jnk, cytosolic or nuclear, could have an effect on their migration as well. They found that knockdown of cytoplasmic JNK enhanced and knockdown of nuclear migration slowed the migration of excitatory neurons (Westerlund et al., 2011). Therefore, Jnk1 may function to decrease migration and Jnk2 might enhance migration during normal cortical development and there by mediate temporal aspects of cortical plate development. Cortical interneurons use cues from their environment, which includes other migratory neuronal 
populations, in order to successfully navigate the cortical rudiment. Genetic ablation of Jnk1 or Jnk2 could not only disrupt radial migration, but also alter temporal aspects of cortical interneuron migration. Additionally, our work suggests that combinatorial loss of Jnk2 and Jnk3 from either radially migrating neurons or from interneurons could affect either population (Chapter 3). Future work should focus on not only the loss of Jnk from interneurons, but also combinatorial loss of Jnk from excitatory neurons. It is unclear whether the loss of Jnk2 and Jnk3 in our mutant cortices at E15.5 contributes to the disruption in columnar architecture and also the radial glial structure or if the loss of Jnk1 from interneurons in combination with the constitutive loss of Jnk2 and Jnk3 causes the disruption to migrating excitatory neurons and their scaffolding.

Reelin is a glycoprotein secreted by Cajal-Retzius cells that prevents the entrance of migrating neurons into the $\mathrm{MZ}$ region and promotes the inside-out organization of excitatory neurons in the cerebral cortex (D'Arcangelo et al., 1995, 1997). Defects in the reelin pathway are known to cause epilepsy and lissencephaly among other severe neurological disorders (D'Arcangelo et al., 1995; Trommsdorff et al., 1999). The reelin pathway is activated by several extracellular receptors including ApoER2 and carried out by intracellular proteins such as Dab1 (Trommsdorff et al., 1999). ApoER2 recruits JNK interacting proteins (JIPs) to form a complex with JNK and with the intracellular proteins, Dab1 (Stockinger et al., 2000). Therefore, reelin could potentially activate JNK within migrating excitatory neurons in the developing cortex. Further, it is unknown whether the JNK-deficient mice express normal levels of reelin, but even if they do, the loss or disruption to JNK signaling in the JNK-deficient mutant mice could impair reelin signaling in the cTKO cortices, which could explain why we see disruption to the architecture of the cortical plate.

Forthcoming experiments should examine whether JNK signaling is required autonomously or non-autonomously for both interneurons and excitatory neuron migration. Further investigation 
into the role of JNK signaling within migrating excitatory neurons might hint at an autonomous role for JNK within migrating excitatory neurons. If JNK signaling is required for excitatory neuron migration and is disrupted, this could potentially disrupt cortical interneuron migration in a non-autonomous manner.

\section{Experiments:}

Conditional triple knockout mice

- To determine the autonomous requirements for JNK for either cortical interneurons or excitatory neurons and the impact that eliminating JNK might subsequently have on the migration of the other cell population, we can create a conditional single, double, and triple mutant mice for Jnk1, Jnk2 and Jnk3 and breed in different cre lines to eliminate JNK in cortical inhibitory neurons (DIx5/6-CIE, Somatostatin-cre, 5HT3aR-cre) and excitatory neurons (Emx1-cre). This would be important for understanding their role within cortical interneurons and allow us to resolve how the of loss of JNK signaling within a certain type of neuron impacts the surrounding environment.

Slice culture transplant assays

- In order to test whether JNK-deficient interneurons can modify their environment, we can place JNK-deficient interneurons on to wildtype cortices to examine whether JNK signaling is required cell autonomously for cortical interneuron migration and whether JNK-deficient interneurons can alter the radial scaffolding in the host tissue, nonautonomously altering radial migration.

- Further, the reverse transplant experiment can be performed to determine if there are non-autonomous requirements for JNK signaling that influence cortical interneuron migration. DIx5/6-CIE wildtype cortical interneurons will be added to JNK-deficient 
cortices to determine if a JNK-deficient environment influences cortical interneuron stream formation and orientation.

- $\quad$ To determine the non-autonomous threshold for JNK signaling in both JNK-deficient interneurons and excitatory neurons, we will introduce JNK-deficient interneurons to JNK-deficient cortices. We can investigate the Jnk genes that are required by both interneurons and excitatory neurons non-autonomously to migrate properly within the developing cortex. If normal migration cannot be attained, we can document the phenotypes that are associated with loss of different Jnk genes in interneurons and excitatory neurons.

Electroporation to label radial glia

- $\quad$ In order to visualize and characterize radial glial morphology in our JNK-deficient cortices, we can electroporate a construct to label brain lipid binding protein (BLBP) in single, double, and triple mutants (Fen et al., 1994; Guo and Anton, 2014). Based on the morphology of radial glial cells labeled with an antibody for Nestin in our cTKO cortices, it is possible that we will see detached radial glial cell endfeet from the basement membrane or radial glial endfeet that do not reach the pial surface, and abnormal radial glial architecture and branching. Further, we could use the BLBP construct to label radial glia in the slice culture transplant assay (mentioned above) to visualize the behavior of the radial glia interacting with cTKO cortical interneurons in wild-type tissue.

Immunocytochemistry

- Antibody labeling for other cortical interneuron markers at E15.5 such as somatostatin

- Somatostatin neurons are primarily known to migrate within the $\mathrm{MZ}$ stream during development and are part of the first cohort to enter the cerebral cortex. 
Therefore, it would be beneficial to see if somatostatin positive neurons are present in cortical interneuron clusters.

- Immunolabeling for markers of reelin in JNK-deficient tissue to look for ectopic or absent expression at E15.5

- Examine markers for radial glial endfeet and basal lamina at the pial surface such as BLBP, laminin, or cadherin at E15.5

- Expand time series to later time points (postnatal) to look at the consequences of the disruptions to radial glia and cortical plate

- Look for ectopic interneurons using antibodies for parvalbumin, somatostatin, VIP

- Immuno-label for layer markers to see if layers include the correct cell types

Breed new cre driver lines into triple knockout line

- In order to test if different cohorts of cortical interneurons require Jnk1 in the same manner and behave the same when deleted in combination with other Jnk genes, we will use cre driver lines to knockout Jnk1 and visualize different subsets of cortical interneurons. We can continue to examine E15.5 using different cre driver lines that will knockout Jnk1 in different subsets of cortical interneurons such as somatostatin or 5HT3R to understand if the same phenotype as the DIx5/6-CIE emerges in the triple knockouts. Using different cre driver lines will help us characterize the behavior of the ectopic cortical interneurons that aggregate in the cortical plate because the labeled cohorts will label fewer cortical interneurons.

Western blot for expression of reelin pathway proteins

- In order to determine if JNK-deficient mice express normal amounts of reelin and if the downstream intracellular proteins within the reelin pathway like Dab1 are expressed at normal levels we could run a western blot using single, double, and triple DIx5/6-CIE 
positive Jnk mutant cortical tissue. We could use cell sorting to separate Dlx5/6-CIE expressing cells (interneurons) and DIx5/6-CIE non-expressing cells (all other cortical cells) and examine the level of the protein components of the reelin pathway in each cohort.

Previous studies suggest an autonomous requirement for JNK signaling through the CXCR4 pathway. The CXCR4/SDF-1 pathway is known to guide cortical interneurons within their migratory streams (Liapi et al., 2008; López-Bendito et al., 2008; Wang et al., 2011; Meechan et al., 2012; Abe et al., 2014). In the absence of this pathway cortical interneurons are known to disperse from migratory streams and layer in the cortical plate (Wang et al., 2011). CXCR4 can activate JNK signaling in breast cancer cells to promote invasion in response to SDF-1 (Serrati et al., 2008). However, there is currently no link between JNK signaling and Cxcr4 in cortical interneurons. SDF-1 has been shown to modify the cytoskeleton through changes in Dcx (Lysko et al., 2014). Increased SDF-1 stabilizes microtubules and promotes tangential migration for cortical interneurons. In the absence of SDF-1, microtubules destabilize, which leads to an increase in branching to promote the exit of cortical interneurons from their migratory streams (Lysko et al., 2014). JNK signaling is known to modify changes within the cytoskeleton through Dcx to increase or decrease branching in PC12, N2A, and primary cerebellar neuron cultures (Gdalyahu et al., 2004). Our cDKOs of Jnk1 and Jnk2 show changes in the distribution of JNKphosphorylated Dcx from the leading process to the soma, where it might destabilize microtubules and lead to increased branching (Appendix Figure A4). At E13.5, we found that cortical interneurons in the cDKOs showed increased branching and spreading of streams. Future studies should focus on Cxcr4's activation of JNK and downstream regulation of Dcx as this may underlie the stream dispersion we see during pharmacological inhibition and in-vivo genetic deletion of JNK. JNK signaling levels have not been studied in CXCR4 mutant cortices. Therefore, future studies should look at Cxcr4 expression in Jnk mutants and Jnk expression in 
Cxcr4 mutants to investigate if there is a connection between JNK and Cxcr4 that regulates stream integrity through downstream activation by JNK.

\section{Experiments:}

Evaluate JNK expression in Cxcr4 mutants

- In order to test if JNK signaling is regulated by Cxcr4, we can breed Cxcr4 mutant mice with DIX5/6-CIE and sort cortical interneurons from respective cortices to look at JNK expression in the absence of Cxcr4 signaling

- Additionally, we can look at the distribution of downstream targets within cortical interneurons especially Dcx and Jnk-phosphorylated Dcx using antibody labeling as well as examine the expression levels of Jnk-phosphorylated Dcx through cell sorting.

Evaluate expression of Cxcr4, SDF-1, and Dcx in Jnk mutants

- In order to evaluate whether the expression of Cxcr4 is altered in JNK-deficient cortical interneurons, we can antibody label for $\mathrm{Cxcr} 4$ in single, double, and triple mutant cortices to look for up or down regulation of Cxcr4 and to look for distribution changes within the cortical interneurons. Additionally, we can sort DIx5/6-CIE cortical interneurons and look at changes in expression of $\mathrm{Cxcr} 4$.

- In-situ hybridization to look at expression of SDF-1 in single, double, and triple mutant cortices

- Antibody labeling for Dcx and Jnk-phosphorylated Dcx in single, double, and triple mutant cortices and cell sorting comparing the expression of Dcx and Jnkphosphorylated Dcx between DIx5/6-CIE positive cortical interneurons and DIx5/6-CIE negative tissue.

Electroporation to label Dcx 
- Create a pCIG3-mCherry-Dcx fusion plasmid that will be electroporated into DIx5/6 or JNK-deficient slices at embryonic day 13.5. This will allow us to simultaneously label interneurons with cytoplasmic GFP and visualize the subcellular localization of redfluorescent Dcx within migrating interneurons. In our single, double, and triple Jnk mutants we will look to see if the localization of Jnk-phosphorylated Dcx has shifted from the leading process to another location within cortical interneurons such as the cell body like we have found in our cDKOs.

Last of all, calcium signaling in migrating neurons is extremely important for nucleokinesis and might modulate portions of the cytoskeleton (Moya and Valdeolmillos, 2004). Although calcium signaling has not been extensively examined in our Jnk mutants, there is a decrease in calbindin labeling in cortical interneurons during early development in cTKO cortices (Appendix Figure A5). Calbindin labeling is confined primarily to the cell body of labeled cortical interneurons, and absent from axonal fibers extending from the developing cortical plate (Appendix Figure A5). Calbindin acts within cortical interneurons as a calcium buffer by sequestering free calcium (Baimbridge et al., 1992). Lower expression of calbindin could indicate that JNK signaling could influence the availability of calcium within cortical interneurons. Intracellular calcium is important for nucleokinesis (Moya and Valdeolmillos, 2004). Increases in calcium signaling near the proximal end of the leading process was shown to co-localize with tubulin and suggested to interact with Dcx through activation of calcium/calmodulin kinases (Moya and Valdeolmillos, 2004). Previous research indicates that calcium/calmodulin kinases can activate the MAPK pathway specifically the JNK pathway suggesting that increased calcium could signal through JNK to influence cytoskeletal architecture within the leading process (Enslen et al., 1996; Kim and Sharma, 2004). Further, calcium transient modulation within cortical interneurons has been linked to the expression of the potassium-chloride transporter, $\mathrm{KCC}$, and $\mathrm{GABA}_{\mathrm{A}}$ and $\mathrm{AMPA} / \mathrm{NMDA}$ receptor mediated depolarization (Bortone and Polleux, 2009). Migrating cortical interneurons were found to exhibit frequent calcium transients during 
knockdown of KCC2 in 2D interneuron cultures (Bortone and Polleux, 2009). Moreover, these calcium transients were further mediated by the expression of $\mathrm{GABA}_{\mathrm{A}}$ and AMPA/NMDA receptors through L-type voltage sensitive calcium channels (Bortone and Polleux, 2009). $\mathrm{GABA}_{\mathrm{A}}$ and $\mathrm{AMPA} / \mathrm{NMDA}$ receptor depolarization and knockdown of KCC2 lead to increased cortical interneuron motility and migration (Bortone and Polleux, 2009). Conversely, increased expression of $\mathrm{KCC} 2$ and decreased $\mathrm{GABA}_{A}$ and AMPA/NMDA receptor depolarization lead to decreased cortical interneuron movement (Bortone and Polleux, 2009). If the cortical interneurons in cTKO cortices fail to appropriately utilize calcium, these studies could explain why we see cortical interneurons aggregating within the cortical plate. This research suggests that JNK could influence cortical interneuron migration (motility and speed) through calcium signaling and could do so by influencing the expression of KCC2, GABA $A_{A}$, and AMPA/NMDA receptors and regulating the cytoskeleton through activation by calcium/ calmodulin kinases.

\section{Experiments:}

Live imaging of MGE explants with a calcium sensor

- In order to understand calcium dynamics in the JNK-deficient cortical interneurons, MGE explants can be grown in collagen and/or Matrigel. DIx5/6-CIE wild-type cortical interneuron MGE explant cultures will be used as controls to compare the calcium dynamics in cDKO and cTKO explants. Fura-2, a calcium sensing dye, will be added to the explants to visualize movement of calcium in cortical interneurons migrating away from the explant. Explants will be imaged live on a Zeiss 710 confocal microscope and cortical interneuron calcium dynamics, motility, and branching dynamics will be assessed via Bitplane's Imaris software. 
Calcium modulation of the cytoskeleton in the absence of JNK

- Use photo-activatable JNK inhibitors to remove JNK signaling from cortical interneurons migrating from MGE explants in conjunction with the addition of Fura-2 to see if there is a change in calcium distribution within migrating cortical interneurons while imaging the explants live on a confocal. When imaging is complete immuno-label for cytoskeletal components, like Dcx and tubulin, which might have been altered with the removal of JNK.

Expression of KCC2, GABAA, and AMPA/NMDA receptors

- In order to determine if the expression levels of KCC2 transporter, GABA $A_{A}$, and AMPA/NMDA receptors could be the reason we see aggregation of cortical interneurons within the cortical plate, we will use cell sorting to assess the transporter and receptor expression levels in DIx5/6-CIE, cDKO, and cTKO in cortical interneurons by comparing the DIx5/6-CIE positive fractions.

\section{Final Thoughts}

A wide variety of neurodevelopmental disorders impacting cortical circuitry have been ascribed to deficits in GABAergic interneurons. The recognition of disorders affecting the assembly and function of inhibitory GABAergic interneuron circuitry has increased in the past decade. It is still unclear how cortical interneurons use and process guidance cues to migrate, organize into laminae, and make connections with surrounding neurons. Therefore, it is imperative that researchers continue to investigate the underlying pathologies beginning with early embryonic development. In this work, we have identified a neurodevelopmental role for the JNK signaling pathway in regulating and mediating cortical interneuron entry into the cerebral cortex, as well as maintaining stream integrity. Although downstream mechanisms are yet to be determined, future work will have major impacts on how we understand the pathology of each of these 
diseases and will build on and connect previous research together linking pathways that are already implicated in causing neurological disease. Pinpointing the exact role of JNK signaling in cortical interneuron migration will give researchers and medical professionals a better understanding of the underlying causes of cortical disorders like autism, schizophrenia, and epilepsy, as well as create avenues for development of further treatment of these disorders. 
Appendix 

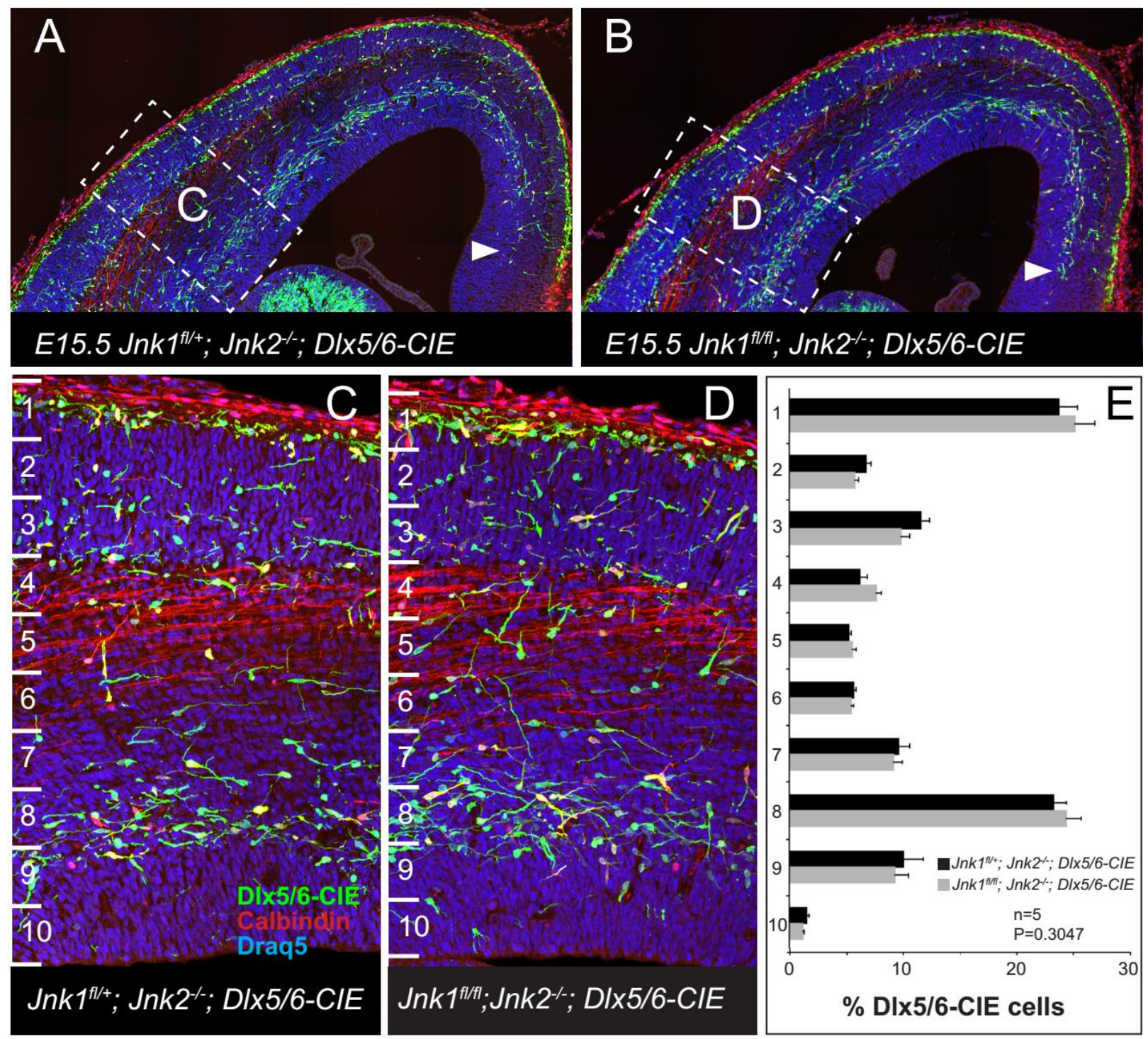

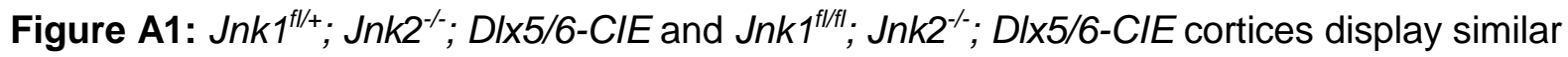
advancement and radial distribution at E15.5. Although, interneuron delays were previously

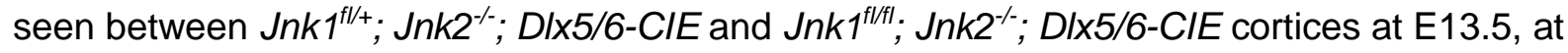
E15.5, the advancement of cortical interneurons seems to even out (A solid arrow; B, solid arrow). Further, the radial distribution of cortical interneurons in $J n k 1^{f l /+} ; \mathrm{Jnk} 2^{-\alpha} ; D / \times 5 / 6-C I E$ (C) and Jnk $1^{f / f t} ;$ Jnk2 ${ }^{-/} ;$DIx5/6-CIE (D) cortices is not different. Quantification of the radial distribution of cortical interneurons revealed no statistically significant differences (E). 

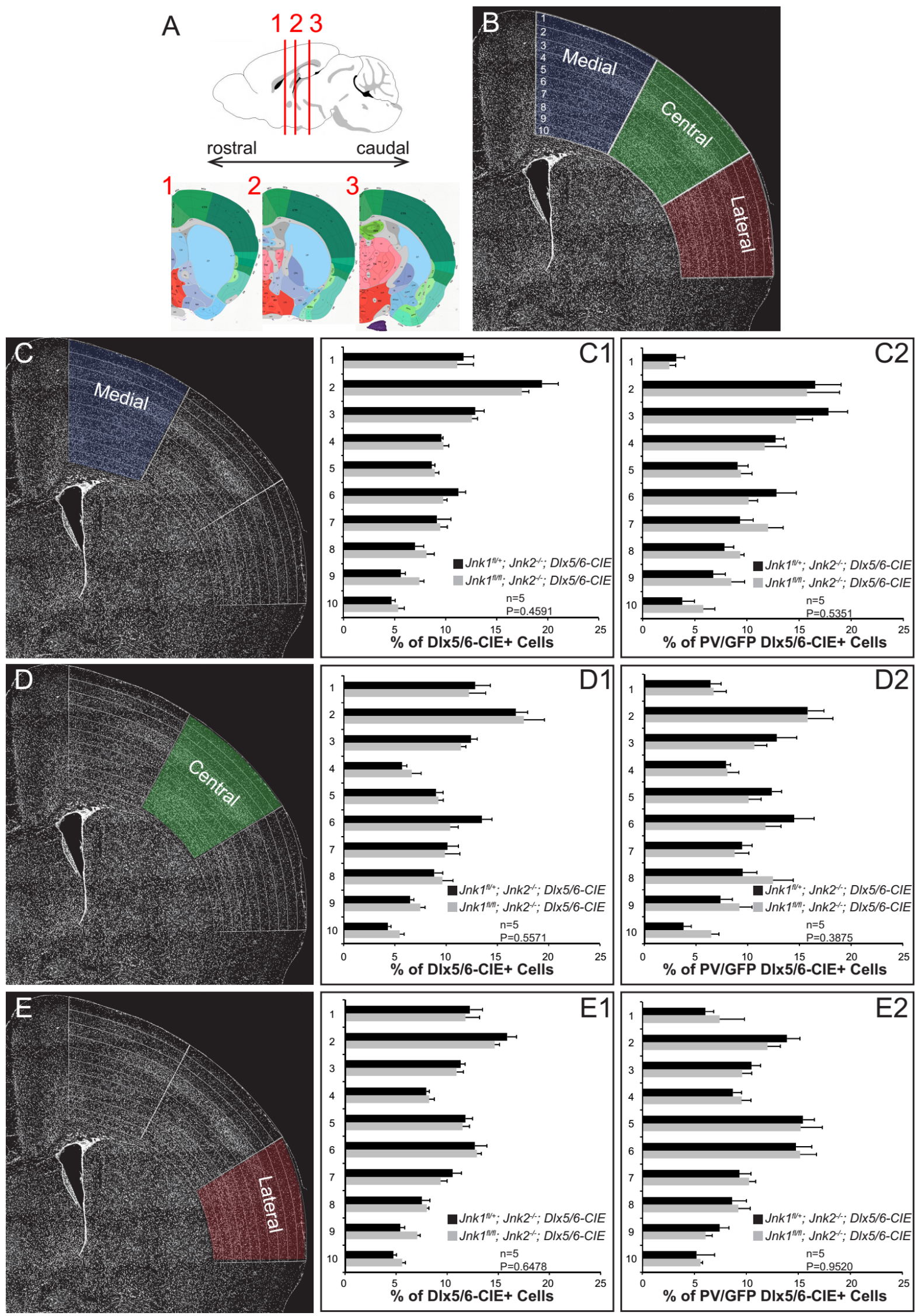
Figure A2: Young adult Jnk $1^{f / /} ; J n k 2^{--} ;$DIX5/6-CIE and $J n k 1^{f l / f l} ; J n k 2^{--} ;$DIX5/6-CIE cortices share similar distributions of cortical interneurons. Sections from three $(\mathbf{A}, \mathbf{1}-\mathbf{3})$ rostro-caudal levels were stained, imaged, and quantified from postnatal day $(P) 21$ brains. Cortices were divided into a medial, central, and lateral partition (B). Each partition was equidistantly binned from the pial surface to the corpus callosum (B). The distribution of DIx5/6-CIE positive cortical

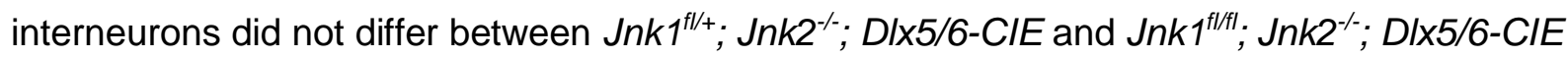
cortices in medial, central, or lateral partitions (C1, D1, E1). Parvalbumin cortical interneurons expressing DIx5/6-CIE showed a similar distribution to the DIx5/6-CIE positive population of cortical interneurons and no differences were found in the medial, central, and lateral partitions (C2, D2, E2). 

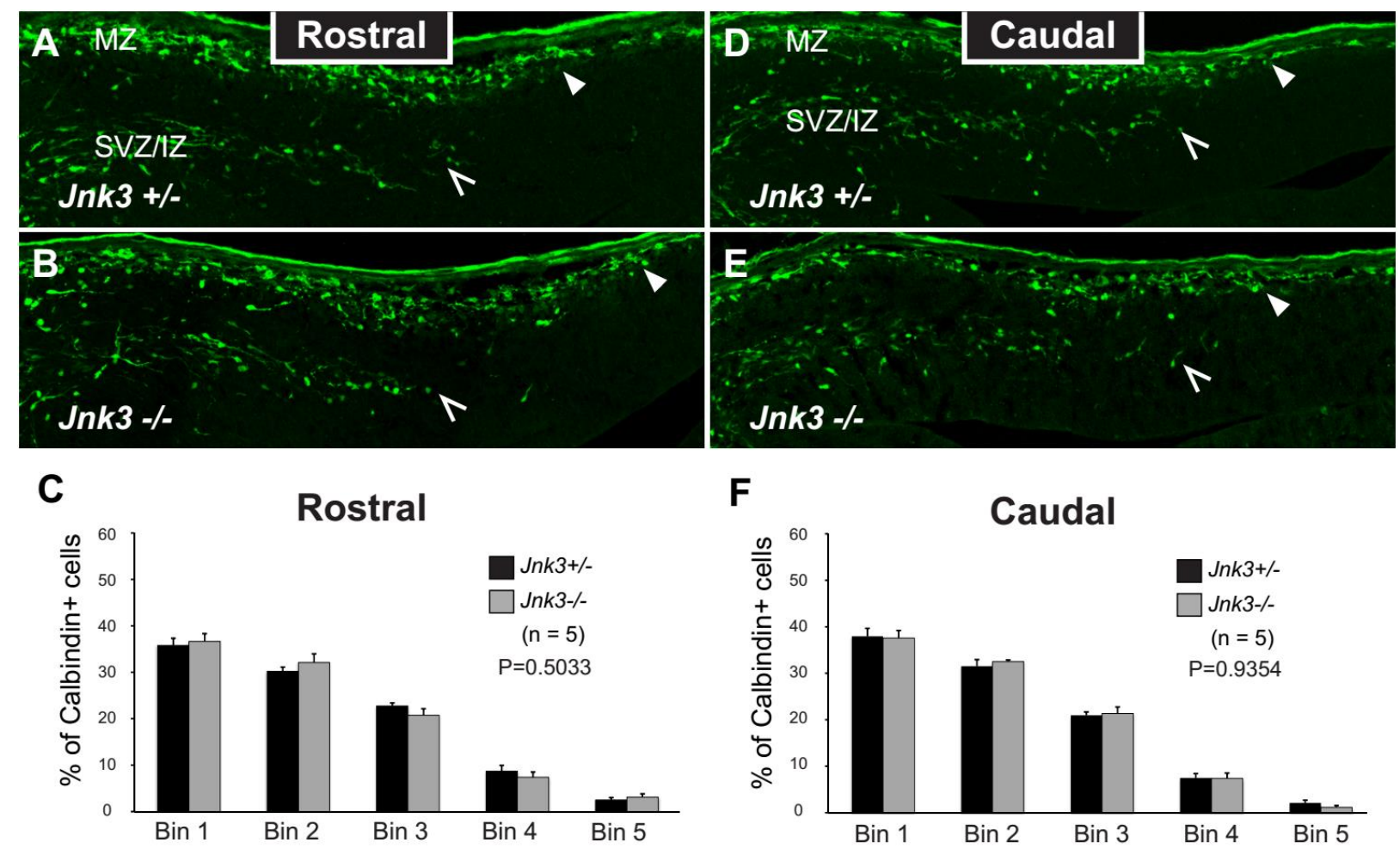

Figure A3: Similar to Jnk2, genetic ablation of Jnk3 at E13.5 does not disrupt cortical interneuron migration. The advancement of calbindin labeled cortical interneurons did not differ between the $\mathrm{Jnk3}^{+/}(\mathrm{A}, \mathrm{D})$ and the $\mathrm{Jnk3^{- \sim }}(\mathrm{B}, \mathrm{E})$ at the rostral or caudal locations. Quantification of the advancement of calbindin positive cortical interneurons showed no statistical differences between the $\mathrm{Jnk}^{+/}$and $\mathrm{Jnk}^{-/}$in the rostral (C) and caudal locations (F). 


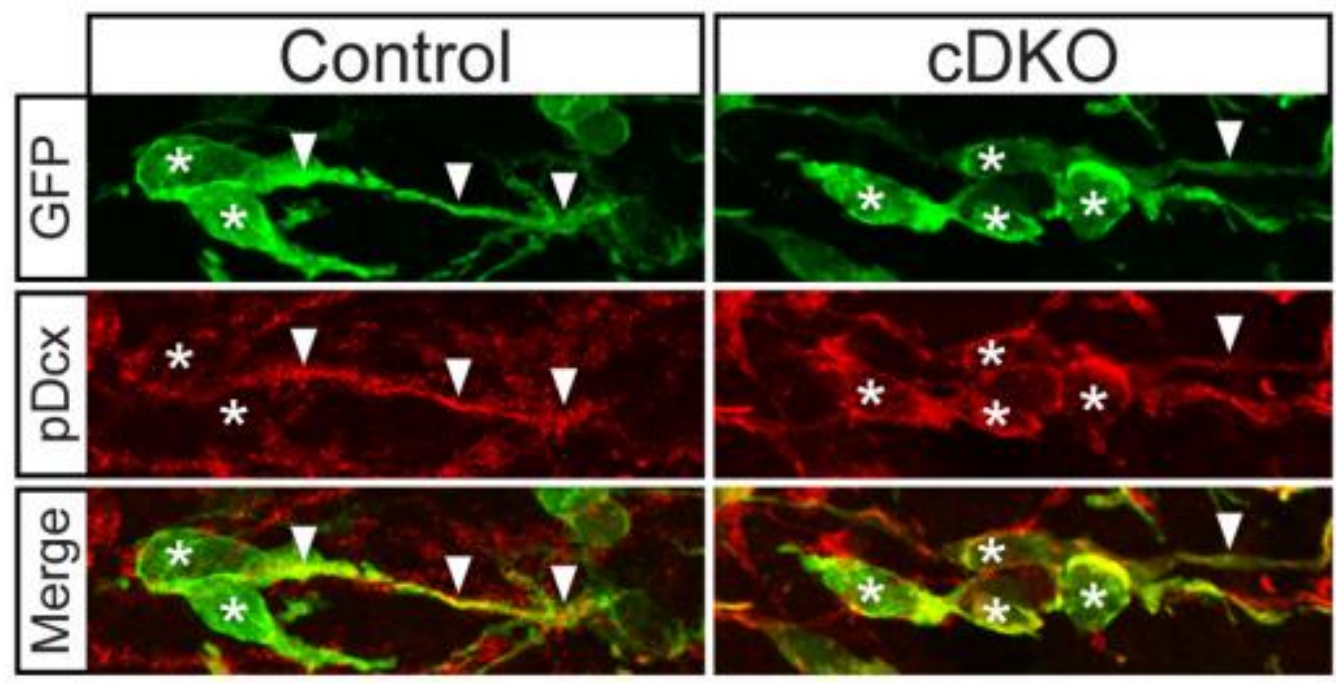

Figure A4: Jnk-phosphorylated Dcx labeling is shifted to the cell body in cortical interneurons in Jnk1 $1^{f l / f l} ;$ Jnk2-1-; DIx5/6-CIE cortices at E13.5. Cortical interneurons migrating within the SVZ stream primarily express Jnk-phosphorylated Dcx in their leading process (middle left). However, in the absence of Jnk1 and 2, Jnk-phosphorylated Dcx heavily labels the cell bodies of SVZ stream cortical interneurons (middle right). 

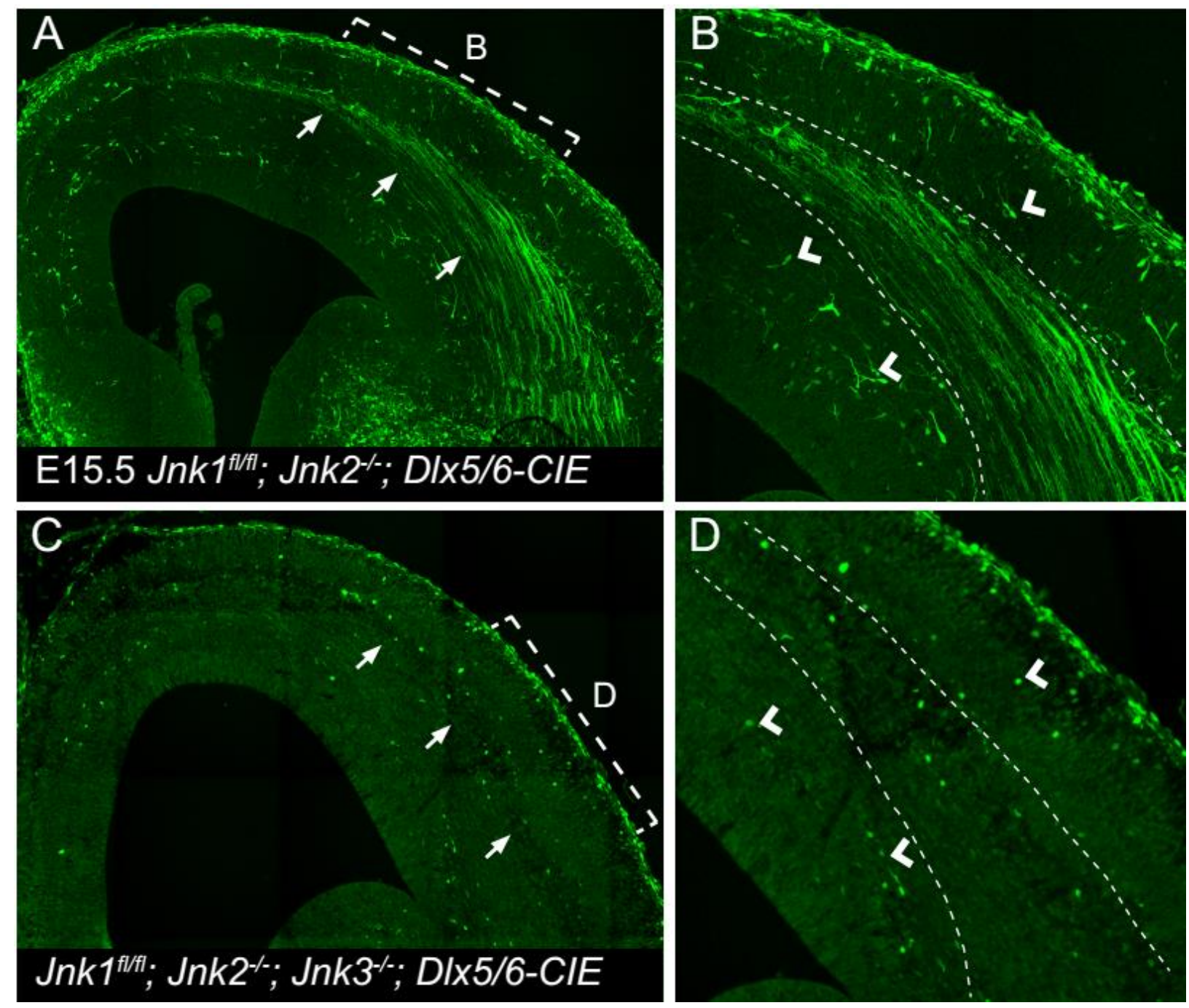

Figure A5: Calbindin labeling is reduced in cortical interneurons and absent in axon projections

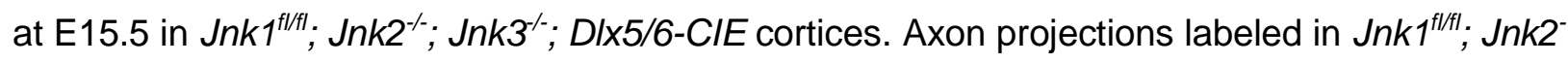

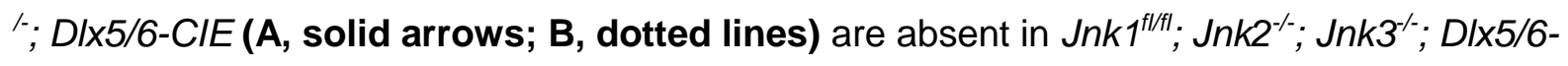

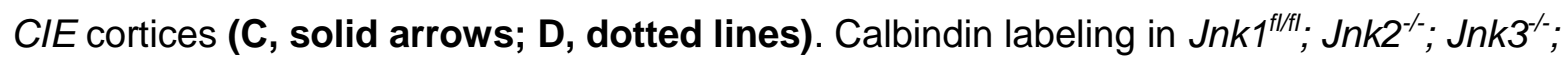
DIx5/6-CIE cortical interneurons is limited to the cell body with sparse labeling of leading processes (D, open arrows) unlike $J n k 1^{f / f l} ; J n k 2^{--} ; D I \times 5 / 6-C I E$ cortices where the majority of cortical interneuron leading processes are labeled (B, open arrows). 


\section{References}

Abe P, Mueller W, Schütz D, Mackay F, Thelen M, Zhang P, Stumm R (2014) CXCR7 prevents excessive CXCL12-mediated downregulation of CXCR4 in migrating cortical interneurons. Development 141:1857-1863.

Akakin D, Martinez-Diaz H, Chen H-X, Roper SN (2012) Reduced densities of parvalbumin- and somatostatin-expressing interneurons in experimental cortical dysplasia and heterotopia in early postnatal development. Epilepsy Res 104:226-233.

Anderson SA, Eisenstat DD, Shi L, Rubenstein JL (1997) Interneuron migration from basal forebrain to neocortex: dependence on Dlx genes. Science (80- ) 278:474-476.

Anderson SA, Marín O, Horn C, Jennings K, Rubenstein JLR (2001) Distinct cortical migrations from the medial and lateral ganglionic eminences. Development 128:353-363.

Angevine J, Sidman R (1961) Autoradiographic study of cell migration during histogenesis of cerebral cortex in the mouse. Nature 192:766-768.

Antypa M, Faux C, Eichele G, Parnavelas JG, Andrews WD (2011) Differential gene expression in migratory streams of cortical interneurons. Eur J Neurosci 34:1584-1594.

Ascoli GA et al. (2008) Petilla terminology: nomenclature of features of GABAergic interneurons of the cerebral cortex. Nat Rev Neurosci 9:557-568.

Baimbridge KG, Celio MR, Rogers JH (1992) Calcium-binding proteins in the nervous system. Trends Neurosci 15:303-308.

Barker RJ, Gourdie RG (2002) JNK Bond Regulation: Why Do Mammalian Hearts Invest in Connexin43? Circ Res 91:556-558.

Baudoin J-P, Viou L, Launay P-S, Luccardini C, Espeso Gil S, Kiyasova V, Irinopoulou T, Alvarez C, Rio J-P, Boudier T, Lechaire J-P, Kessaris N, Spassky N, Métin C (2012) Tangentially migrating neurons assemble a primary cilium that promotes their reorientation to the cortical plate. Neuron 76:1108-1122.

Bayer SA, Altman J, Russo RJ, Dai X, Simmons JA (1991) Cell migration in the rat embryonic neocortex. J Comp Neurol 307:499-516.

Bellion A, Baudoin J-P, Alvarez C, Bornens M, Métin C (2005) Nucleokinesis in tangentially migrating neurons comprises two alternating phases: forward migration of the Golgi/centrosome associated with centrosome splitting and myosin contraction at the rear. J Neurosci 25:5691-5699.

Benes FM, McSparren J, Bird ED, SanGiovanni JP, Vincent SL (1991) Deficits in small interneurons in prefrontal and cingulate cortices of schizophrenic and schizoaffective patients. Arch Gen Psychiatry 48:996-1001.

Bennett BL, Sasaki DT, Murray BW, O'Leary EC, Sakata ST, Xu W, Leisten JC, Motiwala A, Pierce S, Satoh Y, Bhagwat SS, Manning AM, Anderson DW (2001) SP600125, an anthrapyrazolone inhibitor of Jun N-terminal kinase. Proc Natl Acad Sci U S A 98:1368113686.

Björkblom B, Padzik A, Mohammad H, Westerlund N, Komulainen E, Hollos P, Parviainen L, 
Papageorgiou AC, Iljin K, Kallioniemi $\mathrm{O}$, Kallajoki M, Courtney MJ, Mågård M, James $\mathrm{P}$, Coffey ET (2012) C-Jun N-terminal kinase phosphorylation of MARCKSL1 determines actin stability and migration in neurons and in cancer cells. Mol Cell Biol 32:3513-3526.

Boldajipour B, Mahabaleshwar H, Kardash E, Reichman-Fried M, Blaser H, Minina S, Wilson D, Xu Q, Raz E (2008) Control of Chemokine-Guided Cell Migration by Ligand Sequestration. Cell 132:463-473.

Bortone D, Polleux F (2009) KCC2 Expression Promotes the Termination of Cortical Interneuron Migration in a Voltage-Sensitive Calcium-Dependent Manner. Neuron 62:53-71.

Bowman AB, Kamal A, Ritchings BW, Philp AV, McGrail M, Gindhart JG, Goldstein LSB (2000) Kinesin-dependent axonal transport is mediated by the sunday driver (SYD) protein. Cell 103:583-594.

Bozzi Y, Casarosa S, Caleo M (2012) Epilepsy as a neurodevelopmental disorder. Front Psychiatry 3:1-14.

Brecht S, Kirchhof R, Chromik A, Willesen M, Nicolaus T, Raivich G, Wessig J, Waetzig V, Goetz M, Claussen M, Pearse D, Kuan CY, Vaudano E, Behrens A, Wagner E, Flavell RA, Davis RJ, Herdegen T (2005) Specific pathophysiological functions of JNK isoforms in the brain. Eur J Neurosci 21:363-377.

Butt SJB, Fuccillo M, Nery S, Noctor S, Kriegstein A, Corbin JG, Fishell G (2005) The temporal and spatial origins of cortical interneurons predict their physiological subtype. Neuron 48:591-604.

Cai Y, Zhang Q, Wang C, Zhang Y, Ma T, Zhou X, Tian M, Rubenstein JLR, Yang Z (2013) Nuclear receptor COUP-TFII-expressing neocortical interneurons are derived from the medial and lateral/caudal ganglionic eminence and define specific subsets of mature interneurons. J Comp Neurol 521:479-497.

Calderon de Anda F, Rosario AL, Durak O, Tran T, Gräff J, Meletis K, Rei D, Soda T, Madabhushi R, Ginty DD, Kolodkin AL, Tsai L-H (2012) Autism spectrum disorder susceptibility gene TAOK2 affects basal dendrite formation in the neocortex. Nat Neurosci 15:1022-1031.

Campbell AW (1905) Histological studies on the localisation of cerebral function. Cambridge: Cambridge University Press.

Campbell K, Olsson M, Björklund A (1995) Regional incorporation and site-specific differentiation of striatal precursors transplanted to the embryonic forebrain ventricle. Neuron 15:1259-1273.

Canuet L, Ishii R, Iwase M, Ikezawa K, Kurimoto R, Takahashi H, Currais A, Azechi M, Aoki Y, Nakahachi T, Soriano S, Takeda M (2011) Psychopathology and working memory-induced activation of the prefrontal cortex in schizophrenia-like psychosis of epilepsy: Evidence from magnetoencephalography. Psychiatry Clin Neurosci 65:183-190.

Carboni S, Boschert U, Gaillard P, Gotteland J-P, Gillon J-Y, Vitte P-A (2008) AS601245, a cJun NH2-terminal kinase (JNK) inhibitor, reduces axon/dendrite damage and cognitive deficits after global cerebral ischaemia in gerbils. Br J Pharmacol 153:157-163.

Caviness VJ (1976) Patterns of cell and fiber distribution in the neocortex of the reeler mutant mouse. J Comp Neurol 170:435-447. 
Chang L, Jones Y, Ellisman MH, Goldstein LSB, Karin M (2003) JNK1 is required for maintenance of neuronal microtubules and controls phosphorylation of microtubuleassociated proteins. Dev Cell 4:521-533.

Chédotal A, Rijli FM (2009) Transcriptional regulation of tangential neuronal migration in the developing forebrain. Curr Opin Neurobiol 19:139-145.

Cho RY, Konecky RO, Carter CS (2006) Impairments in frontal cortical gamma synchrony and cognitive control in schizophrenia. Proc Natl Acad Sci U S A 103:19878-19883.

Chu J, Anderson SA (2015) Development of cortical interneurons. Neuropsychopharmacology 40:16-23.

Coffey ET (2014) Nuclear and cytosolic JNK signalling in neurons. Nat Rev Neurosci 15:285299.

Curley AA, Arion D, Volk DW, Asafu-Adjei JK, Sampson AR, Fish KN, Lewis DA (2011) Cortical deficits of glutamic acid decarboxylase 67 expression in schizophrenia: clinical, protein, and cell type-specific features. Am J Psychiatry 168:921-929.

D'Arcangelo G, Miao GG, Chen S-C, Soares HD, Morgan Jl, Curran T (1995) A protein related to extracellular matrix proteins deleted in the mouse mutant reeler. Nature 374:719-723.

D'Arcangelo GD, Nakajima K, Miyata T, Ogawa M, Mikoshiba K, Curran T (1997) Reelin is a secreted glycoprotein recognized by the CR-50 monoclonal antibody. J Neurosci 17:23-31.

Danglot L, Triller A, Marty S (2006) The development of hippocampal interneurons in rodents. Hippocampus 16:1032-1060.

Das M, Jiang F, Sluss HK, Zhang C, Shokat KM, Flavell RA, Davis RJ (2007) Suppression of p53-dependent senescence by the JNK signal transduction pathway. Proc Natl Acad Sci U S A 104:15759-15764.

Dong C, Yang DD, Wysk M, Whitmarsh AJ, Davis RJ, Flavell RA (1998) Defective T cell differentiation in the absence of Jnk1. Science (80- ) 282:2092-2095.

Dutton SB, Makinson CD, Papale LA, Shankar A, Balakrishnan B, Nakazawa K, Escayg A (2012) Preferential inactivation of Scn1a in parvalbumin interneurons increases seizure susceptibility. Neurobiol Dis 49:211-220.

Edmondson JC, Hatten ME (1987) Glial-guided granule neuron migration in vitro: a highresolution time-lapse video microscopic study. J Neurosci 7:1928-1934.

Elias LAB, Turmaine M, Parnavelas JG, Kriegstein AR (2010) Cx43 mediates the tangential to radial migratory switch in ventrally derived cortical interneurons. J Neurosci 30:7072-7077.

Elias LAB, Wang DD, Kriegstein AR (2007) Gap junction adhesion is necessary for radial migration in the neocortex. Nature 448:901-907.

Enslen H, Tokumitsu H, Stork PJS, Davis RJ, Soderling TR (1996) Regulation of mitogenactivated protein kinases by a calcium/calmodulin-dependent protein kinase cascade. Proc Natl Acad Sci U S A 93:10803-10808.

Faux C, Rakic S, Andrews W, Britto JM (2012) Neurons on the move: migration and lamination of cortical interneurons. Neurosignals 20:168-189.

Faux C, Rakic S, Andrews W, Yanagawa Y, Obata K, Parnavelas JG (2010) Differential gene 
expression in migrating cortical interneurons during mouse forebrain development. J Comp Neurol 518:1232-1248.

Favata MF, Horiuchi KY, Manos EJ, Daulerio AJ, Stradley DA, Feeser WS, Van Dyk DE, Pitts WJ, Earl RA, Hobbs F, Copeland RA, Magolda RL, Scherle PA, Trzaskos JM (1998) Identification of a novel inhibitor of mitogen-activated protein kinase kinase. J Biol Chem 273:18623-18632.

Fen L, Hatten ME, Heintz N (1994) Brain lipid-binding protein (BLBP): A novel signaling system in the developing mammalian CNS. Neuron 12:895-908.

Fine SE, Weissman A, Gerdes M, Pinto-Martin J, Zackai EH, McDonald-McGinn DM, Emanuel BS (2005) Autism spectrum disorders and symptoms in children with molecularly confirmed 22q11.2 deletion syndrome. J Autism Dev Disord 35:461-470.

Fino E, Yuste R (2011) Dense inhibitory connectivity in neocortex. Neuron 69:1188-1203.

Flames N, Long JE, Garratt AN, Fischer TM, Gassmann M, Birchmeier C, Lai C, Rubenstein JLR, Marın O (2004) Short- and long-range attraction of cortical GABAergic interneurons by neuregulin-1. Neuron 44:251-261.

Flames N, Marín O (2005) Developmental mechanisms underlying the generation of cortical interneuron diversity. Neuron 46:377-381.

Flanagan JG, Vanderhaeghen P (1998) The Ephrins and Eph Receptors in Neural Development. Annu Rev Neurosci 21:309-345.

Friocourt G, Liu JS, Antypa M, Rakic S, Walsh CA, Parnavelas JG (2007) Both doublecortin and doublecortin-like kinase play a role in cortical interneuron migration. J Neurosci 27:38753883.

Gale NW, Holland SJ, Valenzuela DM, Flenniken A, Pan L, Ryan TE, Henkemeyer M, Strebhardt K, Hirai H, Wilkinson DG, Pawson T, Davis S, Yancopoulos GD (1996) Eph receptors and ligands comprise two major specificity subclasses and are reciprocally compartmentalized during embryogenesis. Neuron 17:9-19.

Gdalyahu A, Ghosh I, Levy T, Sapir T, Sapoznik S, Fishler Y, Azoulai D, Reiner O (2004) DCX, a new mediator of the JNK pathway. EMBO J 23:823-832.

Gill DA, Ramsay SL, Tasker RA (2010) Selective reductions in subpopulations of GABAergic neurons in a developmental rat model of epilepsy. Brain Res 1331:114-123.

Gleeson JG, Allen KM, Fox JW, Lamperti ED, Berkovic S, Scheffer I, Cooper EC, Dobyns WB, Minnerath SR, Ross ME, Walsh C a. (1998) Doublecortin, a brain-specific gene mutated in human X-linked lissencephaly and double cortex syndrome, encodes a putative signaling protein. Cell 92:63-72.

Gleeson JG, Lin PT, Flanagan LA, Walsh CA (1999) Doublecortin Is a Microtubule-Associated Protein and Is Expressed Widely by Migrating Neurons. Neuron 23:257-271.

Glickfeld LL, Roberts JD, Somogyi P, Scanziani M (2009) Interneurons hyperpolarize pyramidal cells along their entire somatodendritic axis. Nat Neurosci 12:21-23.

Godin JD, Thomas N, Laguesse S, Malinouskaya L, Close P, Malaise O, Purnelle A, Raineteau O, Campbell K, Fero M, Moonen G, Malgrange B, Chariot A, Metin C, Besson A, Nguyen L (2012) P27Kip1 is a microtubule-associated protein that promotes microtubule 
polymerization during neuron migration. Dev Cell 23:729-744.

Gopal PP, Simonet JC, Shapiro W, Golden JA (2010) Leading process branch instability in Lis1+/- nonradially migrating interneurons. Cereb Cortex 20:1497-1505.

Greig LC, Woodworth MB, Galazo MJ, Padmanabhan H, Macklis JD (2013) Molecular logic of neocortical projection neuron specification, development and diversity. Nat Rev Neurosci 14:755-769.

Guo J, Anton ES (2014) Decision making during interneuron migration in the developing cerebral cortex. Trends Cell Biol 24:342-351.

Gupta S, Barrett T, Whitmarshl AJ, Cavanagh J, Sluss HK, Derijard B, Davis RJ (1996) Selective interaction of JNK protein kinase isoforms with transcription factors. EMBO J 15:2760-2770.

Haeusgen W, Boehm R, Zhao Y, Herdegen T, Waetzig V (2009) Specific activities of individual c-Jun N-terminal kinases in the brain. Neuroscience 161:951-959.

Hashemi E, Ariza J, Rogers H, Noctor SC, Martínez-Cerdeño V (2016) The Number of Parvalbumin-Expressing Interneurons Is Decreased in the Medial Prefrontal Cortex in Autism. Cereb Cortex:1-13.

Hashimoto T, Arion D, Unger T, Maldonado-Avilés JG, Morris HM, Volk DW, Mirnics K, Lewis D a (2008) Alterations in GABA-related transcriptome in the dorsolateral prefrontal cortex of subjects with schizophrenia. Mol Psychiatry 13:147-161.

Higginbotham H, Eom TY, Mariani LE, Bachleda A, Hirt J, Gukassyan V, Cusack CL, Lai C, Caspary T, Anton ES (2012) Arl13b in primary cilia regulates the migration and placement of interneurons in the developing cerebral cortex. Dev Cell 23:925-938.

Hirai S, Cui DF, Miyata T, Ogawa M, Kiyonari H, Suda Y, Aizawa S, Banba Y, Ohno S (2006) The c-Jun N-terminal kinase activator dual leucine zipper kinase regulates axon growth and neuronal migration in the developing cerebral cortex. J Neurosci 26:11992-12002.

Inan M, Welagen J, Anderson SA (2012) Spatial and temporal bias in the mitotic origins of somatostatin- and parvalbumin-expressing interneuron subgroups and the chandelier subtype in the medial ganglionic eminence. Cereb cortex 22:820-827.

Isaacson JS, Scanziani M (2011) How inhibition shapes cortical activity. Neuron 72:231-243.

Ito-Ishida A, Ure K, Chen H, Swann JW, Zoghbi HY (2015) Loss of MeCP2 in parvalbumin-and somatostatin-expressing neurons in mice leads to distinct Rett syndrome-like phenotypes. Neuron 88:651-658.

Jacob J (2016) Cortical interneuron dysfunction in epilepsy associated with autism spectrum disorders. Epilepsia 57:182-193.

Jin J, Suzuki H, Hirai S-I, Mikoshiba K, Ohshima T (2010) JNK phosphorylates Ser332 of doublecortin and regulates its function in neurite extension and neuronal migration. Dev Neurobiol 70:929-942.

Kappeler C, Saillour Y, Baudoin J-P, Tuy FPD, Alvarez C, Houbron C, Gaspar P, Hamard G, Chelly J, Métin C, Francis F (2006) Branching and nucleokinesis defects in migrating interneurons derived from doublecortin knockout mice. Hum Mol Genet 15:1387-1400.

Karagiannis A, Gallopin T, Dávid C, Battaglia D, Geoffroy H, Rossier J, Hillman EMC, Staiger 
JF, Cauli B (2009) Classification of NPY-expressing neocortical interneurons. J Neurosci 29:3642-3659.

Kato M, Dobyns WB (2005) X-linked lissencephaly with abnormal genitalia as a tangential migration disorder causing intractable epilepsy: proposal for a new term, "interneuronopathy". J Child Neurol 20:392-397.

Kawaguchi $Y$ (1995) Physiological subgroups of nonpyramidal cells with specific morphological characteristics in layer II/III of rat frontal cortex. J Neurosci 15:2638-2655.

Kawaguchi Y, Kubota Y (1993) Correlation of physiological subgroupings of nonpyramidal cells with parvalbumin- and calbindin D28k-immunoreactive neurons in layer $\mathrm{V}$ of rat frontal cortex. J Neurophysiol 70:387-396.

Kawaguchi Y, Kubota Y (1996) Physiological and morphological identification of somatostatinor vasoactive intestinal polypeptide-containing cells among GABAergic cell subtypes in rat frontal cortex. J Neurosci 16:2701-2715.

Kawaguchi Y, Kubota Y (1997) GABAergic cell subtypes and their synaptic connections in rat frontal cortex. Cereb Cortex 7:476-486.

Kawauchi T, Chihama K, Nishimura Y V., Nabeshima Y, Hoshino M (2005) MAP1B phosphorylation is differentially regulated by Cdk5/p35, Cdk5/p25, and JNK. Biochem Biophys Res Commun 331:50-55.

Kim D, Jeong H, Lee J, Ghim J, Her ES, Lee S, Kim D, Jeong H, Lee J, Ghim J, Her ES, Lee S (2016a) Distinct roles of parvalbumin- and somatostatin- expressing interneurons in working memory article distinct roles of parvalbumin- and somatostatin-expressing interneurons in working memory. Neuron 92:902-915.

Kim H, Ährlund-Richter S, Wang X, Deisseroth K, Carlén M (2016b) Prefrontal parvalbumin neurons in control of attention. Cell 164:208-218.

Kim J, Sharma RP (2004) Calcium-mediated activation of c-Jun NH2-terminal kinase (JNK and apoptosis in response to cadmium in murine macrophages. Toxicol Sci 81:518-527.

Kuan C, Yang DD, Roy DRS, Davis RJ, Rakic P, Flavell RA (1999) The Jnk1 and Jnk2 protein kinases are required for regional specific apoptosis during early brain development. Neuron 22:667-676.

Kunde S-A, Rademacher N, Tzschach A, Wiedersberg E, Ullmann R, Kalscheuer VM, Shoichet $S$ a (2013) Characterisation of de novo MAPK10/JNK3 truncation mutations associated with cognitive disorders in two unrelated patients. Hum Genet 132:461-471.

Lavdas AA, Grigoriou M, Pachnis V, Parnavelas JG (1999) The medial ganglionic eminence gives rise to a population of early neurons in the developing cerebral cortex. J Neurosci 19:7881-7888.

Lee S, Hjerling-Leffler J, Zagha E, Fishell G, Rudy B (2010) The largest group of superficial neocortical GABAergic interneurons expresses ionotropic serotonin receptors. J Neurosci 30:16796-16808.

Lewis DA, Curley AA, Glausier JR, Volk DW (2012) Cortical parvalbumin interneurons and cognitive dysfunction in schizophrenia. Trends Neurosci 35:57-67.

Li G, Adesnik H, Li J, Long J, Nicoll RA, Rubenstein JLR, Pleasure SJ (2008) Regional 
distribution of cortical interneurons and development of inhibitory tone are regulated by Cxcl12/Cxcr4 signaling. J Neurosci 28:1085-1098.

Li H, Chou S-J, Hamasaki T, Perez-Garcia CG, O'Leary DDM (2012) Neuregulin repellent signaling via ErbB4 restricts GABAergic interneurons to migratory paths from ganglionic eminence to cortical destinations. Neural Dev 7:10.

Liapi A, Pritchett J, Jones O, Fujii N, Parnavelas JG, Nadarajah B (2008) Stromal-derived factor 1 signalling regulates radial and tangential migration in the developing cerebral cortex. Dev Neurosci 30:117-131.

Lin-Hendel EG, McManus MJ, Wallace DC, Anderson SA, Golden JA (2016) Differential mitochondrial requirements for radially and non-radially migrating cortical neurons: implications for mitochondrial disorders. Cell Rep 15:229-237.

Livak KJ, Schmittgen TD (2001) Analysis of relative gene expression data using real-time quantitative PCR and 2(-Delta DeltaC(T)) method. Methods 25:402-408.

López-Bendito G, Sánchez-Alcañiz JA, Pla R, Borrell V, Picó E, Valdeolmillos M, Marín O (2008) Chemokine signaling controls intracortical migration and final distribution of GABAergic interneurons. J Neurosci 28:1613-1624.

Loukas M, Pennell C, Groat C, Tubbs RS, Cohen-Gadol AA (2011) Korbinian Brodmann (18681918) and his contributions to mapping the cerebral cortex. Neurosurgery 68:6-11.

Lysko DE, Putt M, Golden JA (2011) SDF1 regulates leading process branching and speed of migrating interneurons. J Neurosci 31:1739-1745.

Lysko DE, Putt M, Golden JA (2014) SDF1 reduces interneuron leading process branching through dual regulation of actin and microtubules. J Neurosci 34:4941-4962.

Ma T, Wang C, Wang L, Zhou X, Tian M, Zhang Q, Zhang Y, Li J, Liu Z, Cai Y, Liu F, You Y, Chen C, Campbell K, Song H, Ma L, Rubenstein JL, Yang Z (2013) Subcortical origins of human and monkey neocortical interneurons. Nat Neurosci 16:1588-1597.

Ma T, Zhang Q, Cai Y, You Y, Rubenstein JLR, Yang Z (2012) A subpopulation of dorsal lateral/caudal ganglionic eminence-derived neocortical interneurons expresses the transcription factor Sp8. Cereb Cortex 22:2120-2130.

Ma Y, Hu H, Berrebi AS, Mathers PH, Agmon A (2006) Distinct subtypes of somatostatincontaining neocortical interneurons revealed in transgenic mice. J Neurosci 26:5069-5082.

Marín O (2012) Interneuron dysfunction in psychiatric disorders. Nat Rev Neurosci 13:107-120.

Marín O (2013) Cellular and molecular mechanisms controlling the migration of neocortical interneurons. Eur J Neurosci 38:2019-2029.

Marín O, Valiente M, Ge X, Tsai L-H (2010) Guiding neuronal cell migrations. Cold Spring Harb Perspect Biol 2:1-20.

Marín O, Yaron A, Bagri A, Tessier-Lavigne M, Rubenstein JLR (2001) Sorting of striatal and cortical interneurons regulated by semaphorin-neuropilin interactions. Science (80- ) 293:872-875.

Martini FJ, Valiente M, López Bendito G, Szabó G, Moya F, Valdeolmillos M, Marín O (2009) Biased selection of leading process branches mediates chemotaxis during tangential neuronal migration. Development 136:41-50. 
Meechan DW, Rutz HLH, Fralish MS, Maynard TM, Rothblat LA, Lamantia A-S (2015) Cognitive ability is associated with altered medial frontal cortical circuits in the LgDel mouse model of 22q11.2DS. Cereb cortex 25:1143-1151.

Meechan DW, Tucker ES, Maynard TM, LaMantia A-S (2009) Diminished dosage of 22q11 genes disrupts neurogenesis and cortical development in a mouse model of $22 \mathrm{q} 11$ deletion/DiGeorge syndrome. Proc Natl Acad Sci U S A 106:16434-16445.

Meechan DW, Tucker ES, Maynard TM, LaMantia A-S (2012) Cxcr4 regulation of interneuron migration is disrupted in 22q11.2 deletion syndrome. Proc Natl Acad Sci U S A 109:1860118606.

Meencke HJ, Veith G (1992) Migration disturbances in epilepsy. Mol Neurobiol Epilepsy 9:3139.

Métin C, Baudoin J-P, Rakić S, Parnavelas JG (2006) Cell and molecular mechanisms involved in the migration of cortical interneurons. Eur J Neurosci 23:894-900 Available at: http://www.ncbi.nlm.nih.gov/pubmed/16519654 [Accessed February 9, 2013].

Métin C, Pedraza M (2014) Cilia: traffic directors along the road of cortical development. Neuroscientist 20:468-482.

Meyer HS, Schwarz D, Wimmer VC, Schmitt AC, Kerr JND, Sakmann B, Helmstaedter M (2011) Inhibitory interneurons in a cortical column form hot zones of inhibition in layers 2 and $5 \mathrm{~A}$. Proc Natl Acad Sci 108:16807-16812.

Miyoshi G, Fishell G (2011) GABAergic interneuron lineages selectively sort into specific cortical layers during early postnatal development. Cereb Cortex 21:845-852.

Miyoshi G, Hjerling-Leffler J, Karayannis T, Sousa VH, Butt SJB, Battiste J, Johnson JE, Machold RP, Fishell G (2010) Genetic fate mapping reveals that the caudal ganglionic eminence produces a large and diverse population of superficial cortical interneurons. $J$ Neurosci 30:1582-1594.

Morales M, Battenberg E, De Lecea L, Bloom FE (1996) The type 3 serotonin receptor is expressed in a subpopulation of GABAergic neurons in the rat neocortex and hippocampus. Brain Res 731:199-202.

Morales M, Bloom FE (1997) The 5-HT3 receptor is present in different subpopulations of GABAergic neurons in the rat telencephalon. J Neurosci 17:3157-3167.

Moya F, Valdeolmillos M (2004) Polarized increase of calcium and nucleokinesis in tangentially migrating neurons. Cereb Cortex 14:610-618.

Murphy KC, Jones LA, Owen MJ (1999) High rates of schizophrenia in adults with velo-cardiofacial syndrome. Arch Gen Psychiatry 56:940-945.

Murray AJ, Woloszynowska-Fraser MU, Ansel-Bollepalli L, Cole KLH, Foggetti A, Crouch B, Riedel G, Wulff P (2015) Parvalbumin-positive interneurons of the prefrontal cortex support working memory and cognitive flexibility. Sci Rep 5:1-14.

Myers AK, Meechan DW, Adney DR, Tucker ES (2014) Cortical interneurons require Jnk1 to enter and navigate the developing cerebral cortex. J Neurosci 34:7787-7801.

Nadarajah B, Brunstrom JE, Grutzendler J, Wong ROL, Pearlman AL (2001) Two modes of radial migration in early development of the cerebral cortex. Nat Neurosci 4:143-150. 
Nery S, Fishell G, Corbin JG (2002) The caudal ganglionic eminence is a source of distinct cortical and subcortical cell populations. Nat Neurosci 5:1279-1287.

Niklasson L, Rasmussen P, Óskarsdóttir S, Gillberg C (2009) Autism, ADHD, mental retardation and behavior problems in 100 individuals with 22q11 deletion syndrome. Res Dev Disabil 30:763-773.

Niquille M, Minocha S, Hornung JP, Rufer N, Valloton D, Kessaris N, Alfonsi F, Vitalis T, Yanagawa Y, Devenoges C, Dayer A, Lebrand C (2013) Two specific populations of GABAergic neurons originating from the medial and the caudal ganglionic eminences aid in proper navigation of callosal axons. Dev Neurobiol 73:647-672.

Nishimura Y V., Sekine K, Chihama K, Nakajima K, Hoshino M, Nabeshima Y, Kawauchi T (2010) Dissecting the factors involved in the locomotion mode of neuronal migration in the developing cerebral cortex. J Biol Chem 285:5878-5887.

Nishimura Y V., Shikanai M, Hoshino M, Ohshima T, Nabeshima Y, Mizutani K-I, Nagata K-I, Nakajima K, Kawauchi T (2014) Cdk5 and its substrates, Dcx and p27kip1, regulate cytoplasmic dilation formation and nuclear elongation in migrating neurons. Development 141:3540-3550.

Noctor SC, Flint AC, Weissman TA, Dammerman RS, Kriegstein AR (2001) Neurons derived from radial glial cells establish radial units in neocortex. Nature 409:714-720.

O'Rourke NA, Chenn A, McConnell SK (1997) Postmitotic neurons migrate tangentially in the cortical ventricular zone. Development 124:997-1005.

O'Rourke NA, Sullivan DP, Kaznowski CE, Jacobs AA, McConnell SK (1995) Tangential migration of neurons in the developing cerebral cortex. Development 121:2165-2176.

Oliva Jr AA, Atkins CM, Copenagle L, Banker GA (2006) Activated C-Jun N-terminal kinase is required for axon formation. J Neurosci 26:9462-9470.

Oliva Jr AA, Jiang M, Lam T, Smith KL, Swann JW (2000) Novel hippocampal interneuronal subtypes identified using transgenic mice that express green fluorescent protein in GABAergic interneurons. J Neurosci 20:3354-3368.

Petrich BG, Gong X, Lerner DL, Wang X, Brown JH, Saffitz JE, Wang Y (2002) c-Jun N-terminal kinase activation mediates downregulation of connexin43 in cardiomyocytes. Circ Res 91:640-647.

Polleux F, Anton ES (2005) Neuronal Migration in the Developing Brain. In: Developmental Neurobiology (Rao MS, Jacobson M, eds), pp 223-239. New York: Kluwer Academic/Plenum Publishers.

Polleux F, Ghosh A (2002) The Slice Overlay Assay: A Versatile Tool to Study the Influence of Extracellular Signals on Neuronal Development. Sci STKE 136:pl9.

Polleux F, Whitford KL, Dijkhuizen PA, Vitalis T, Ghosh A (2002) Control of cortical interneuron migration by neurotrophins and PI3-kinase signaling. Development 129:3147-3160.

Poluch S, Juliano SL (2007) A normal radial glial scaffold is necessary for migration of interneurons during neocortical development. Glia 55:822-830.

Powell EM, Mars WM, Levitt P (2001) Hepatocyte growth factor/scatter factor is a motogen for interneurons migrating from the ventral to dorsal telencephalon. Neuron 30:79-89. 
Pozas E, Ibáñez CF (2005) GDNF and GFRa1 promote differentiation and tangential migration of cortical GABAergic neurons. Neuron 45:701-713.

Qu C, Li W, Shao Q, Dwyer T, Huang H, Yang T, Liu G (2013) C-Jun N-terminal kinase 1 (JNK1) is required for coordination of netrin signaling in axon guidance. J Biol Chem 288:1883-1895.

Rakic P (1971) Guidance of neurons migrating to the fetal monkey neocortex. Brain Res 33:471-476.

Rakic $\mathrm{P}$ (1972) Modes of cell migration to the superficial layers of fetal monkey neocortex. J Comp Neurol 145:61-83.

Rakic $P$ (1974) Neurons in rhesus monkey visual cortex: systematic relation between time of origin and eventual disposition. Science (80- ) 183:425-427.

Rakic P (1975) Cell migration and neuronal ectopias in the brain. Birth Defects Orig Artic Ser 11:95-129.

Rakic P (1978) Neuronal migration and contact guidance in the primate telencephalon. Postgrad Med J 54:25-40.

Rudolph J, Zimmer G, Steinecke A, Barchmann S, Bolz J (2010) Ephrins guide migrating cortical interneurons in the basal telencephalon. Cell Adh Migr 4:400-408.

Rudy B, Fishell G, Lee S, Hjerling-Leffler J (2011) Three groups of interneurons account for nearly $100 \%$ of neocortical GABAergic neurons. Dev Neurobiol 71:45-61.

Sabapathy K, Jochum W, Hochedlinger K, Chang L, Karin M, Wagner EF (1999) Defective neural tube morphogenesis and altered apoptosis in the absence of both JNK1 and JNK2. Mech Dev 89:115-124.

Sánchez-Alcañiz JA, Haege S, Mueller W, Pla R, Mackay F, Schulz S, López-Bendito G, Stumm R, Marín O (2011) Cxcr7 controls neuronal migration by regulating chemokine responsiveness. Neuron 69:77-90.

Schwaller B, Tetko I V., Tandon P, Silveira DC, Vreugdenhil M, Henzi T, Potier M-C, Celio MR, Villa AEP (2004) Parvalbumin deficiency affects network properties resulting in increased susceptibility to epileptic seizures. Mol Cell Neurosci 25:650-663.

Serrati S, Margheri F, Fibbi G, Di Cara G, Minafra L, Pucci-Minafra I, Liotta F, Annunziato F, Pucci M, Del Rosso M (2008) Endothelial cells and normal breast epithelial cells enhance invasion of breast carcinoma cells by CXCR-4-dependent up-regulation of urokinase-type plasminogen activator receptor (UPAR , CD87) expression. J Pathol 214:545-554.

Sgadò P, Genovesi S, Kalinovsky A, Zunino G, Macchi F, Allegra M, Murenu E, Provenzano G, Tripathi PP, Casarosa S, Joyner AL, Bozzi Y (2013) Loss of GABAergic neurons in the hippocampus and cerebral cortex of Engrailed-2 null mutant mice: Implications for autism spectrum disorders. Exp Neurol 247:496-505.

Shoichet SA, Duprez L, Hagens O, Waetzig V, Menzel C, Herdegen T, Schweiger S, Dan B, Vamos E, Ropers H-H, Kalscheuer VM (2006) Truncation of the CNS-expressed JNK3 in a patient with a severe developmental epileptic encephalopathy. Hum Genet 118:559-567.

Snijders TM, Milivojevic B, Kemner C (2013) Atypical excitation-inhibition balance in autism captured by the gamma response to contextual modulation. Neurolmage Clin 3:65-72. 
Stancik EK, Navarro-Quiroga I, Sellke R, Haydar TF (2010) Heterogeneity in ventricular zone neural precursors contributes to neuronal fate diversity in the postnatal neocortex. $J$ Neurosci 30:7028-7036.

Stefansson H et al. (2002) Neuregulin 1 and susceptibility to schizophrenia. Am J Hum Genet 71:877-892.

Stenman J, Toresson H, Campbell K (2003) Identification of two distinct progenitor populations in the lateral ganglionic eminence: implications for striatal and olfactory bulb neurogenesis. J Neurosci 23:167-174.

Stockinger W, Brandes C, Fasching D, Hermann M, Gotthardt M, Herz J, Schneider WJ, Nimpf $J$ (2000) The reelin receptor ApoER2 recruits JNK-interacting proteins-1 and -2. J Biol Chem 275:25625-25632.

Stouffer MA, Golden JA, Francis F (2015) Neuronal migration disorders: Focus on the cytoskeleton and epilepsy. Neurobiol Dis 92:18-45.

Stumm R, Kolodziej A, Schulz S, Kohtz JD, Hollt V (2007) Patterns of SDF-1 alpha and SDF-1 gamma mRNAs migration pathways, and phenotypes of CXCR4-expressing neurons in the developing rat telencephalon. J Comp Neurol 502:382-399.

Stumm RK, Zhou C, Ara T, Lazarini F, Dubois-Dalcq M, Nagasawa T, Höllt V, Schulz S (2003) CXCR4 regulates interneuron migration in the developing neocortex. J Neurosci 23:51235130.

Sussel L, Marin O, Kimura S, Rubenstein JLR (1999) Loss of Nkx2.1 homeobox gene function results in a ventral to dorsal molecular respecification within the basal telencephalon: evidence for a transformation of the pallidum into the striatum. Development 126:33593370.

Szabadics J, Varga C, Molnar G, Olah S, Barzo P, Tamas G (2006) Excitatory effect of GABAergic axo-axonic cells in cortical microcircuits. Science (80- ) 311:233-235.

Szentagothai J (1975) The "module-concept" in cerebral cortex architecture. Brain Res 95:475496.

Tahirovic S, Hellal F, Neukirchen D, Hindges R, Garvalov BK, Flynn KC, Stradal TE, ChrostekGrashoff A, Brakebusch C, Bradke F (2010) Rac1 regulates neuronal polarization through the WAVE complex. J Neurosci 30:6930-6943.

Taniguchi H, Lu J, Huang ZJ (2013) The spatial and temporal origin of chandelier cells in mouse neocortex. Science (80- ) 339:70-74.

Tiveron M-C, Rossel M, Moepps B, Zhang YL, Seidenfaden R, Favor J, König N, Cremer H (2006) Molecular interaction between projection neuron precursors and invading interneurons via stromal-derived factor 1 (CXCL12)/CXCR4 signaling in the cortical subventricular zone/intermediate zone. J Neurosci 26:13273-13278.

Tivodar S, Kalemaki K, Kounoupa Z, Vidaki M, Theodorakis K, Denaxa M, Kessaris N, De Curtis I, Pachnis V, Karagogeos D (2015) Rac-GTPases regulate microtubule stability and axon growth of cortical GABAergic interneurons. Cereb Cortex 25:2370-2382.

Toritsuka M, Kimoto S, Muraki K, Landek-Salgado MA, Yoshida A, Yamamoto N, Horiuchi Y, Hiyama H, Tajinda K, Keni N, Illingworth E, Iwamoto T, Kishimoto T, Sawa A, Tanigaki K (2013) Deficits in microRNA-mediated Cxcr4/Cxcl12 signaling in neurodevelopmental 
deficits in a 22q11 deletion syndrome mouse model. Proc Natl Acad Sci U S A 110:1755217557.

Touzot A, Ruiz-reig N, Vitalis T, Studer M (2016) Molecular control of two novel migratory paths for CGE-derived interneurons in the developing mouse brain. Development 143:17531765.

Trommsdorff M, Gotthardt M, Hiesberger T, Shelton J, Stockinger W, Nimpf J, Hammer RE, Richardson JA, Herz J (1999) Reeler/disabled-like disruption of neuronal migration in knockout mice lacking the VLDL receptor and ApoE receptor 2. Cell 97:689-701.

Tucker ES, Polleux F, LaMantia A-S (2006) Position and time specify the migration of a pioneering population of olfactory bulb interneurons. Dev Biol 297:387-401.

Valcanis H, Tan S-S (2003) Layer specification of transplanted interneurons in developing mouse neocortex. J Neurosci 23:5113-5122.

Verhey KJ, Meyer D, Deehan R, Blenis J, Schnapp BJ, Rapoport TA, Margolis B (2001) Cargo of kinesin identified as JIP scaffolding proteins and associated signaling molecules. J Cell Biol 152:959-970.

Vogt D, Cho KKA, Lee AT, Sohal VS, Rubenstein JLR (2015) The parvalbumin/somatostatin ratio is increased in Pten mutant mice and by human PTEN ASD alleles. Cell Rep 11:944956.

Volk DW, Chitrapu A, Edelson JR, Lewis DA (2015) Chemokine receptors and cortical interneuron dysfunction in schizophrenia. Schizophr Res 167:12-17.

Vucurovic K, Gallopin T, Ferezou I, Rancillac A, Chameau P, Van Hooft JA, Geoffroy H, Monyer H, Rossier J, Vitalis T (2010) Serotonin 3A receptor subtype as an early and protracted marker of cortical interneuron subpopulations. Cereb Cortex 20:2333-2347.

Waclaw RR, Wang B, Pei Z, Ehrman LA, Campbell K (2009) Distinct temporal requirements for the homeobox gene Gsx2 in specifying striatal and olfactory bulb neuronal fates. Neuron 63:451-465.

Wang X, Nadarajah B, Robinson AC, McColl BW, Jin J-W, Dajas-Bailador F, Boot-Handford RP, Tournier $C$ (2007) Targeted deletion of the mitogen-activated protein kinase kinase 4 gene in the nervous system causes severe brain developmental defects and premature death. Mol Cell Biol 27:7935-7946.

Wang Y, Li G, Stanco A, Long J, Crawford D, Potter GB, Pleasure SJ, Behrens T, Rubenstein JLR (2011) CXCR4 and CXCR7 have distinct functions in regulating interneuron migration. Neuron 69:61-76.

Westerlund N, Zdrojewska J, Padzik A, Komulainen E, Björkblom B, Rannikko E, Tararuk T, Garcia-Frigola C, Sandholm J, Nguyen L, Kallunki T, Courtney MJ, Coffey ET (2011) Phosphorylation of SCG10/stathmin-2 determines multipolar stage exit and neuronal migration rate. Nat Neurosci 14:305-313.

Whitmarsh AJ, Cavanagh J, Tournier C, Yasuda J, Davis RJ (1998) A mammalian scaffold complex that selectively mediates MAP kinase activation. Science (80- ) 281:1671-1674.

Whitmarsh AJ, Davis RJ (1998) Structural organization of MAP- kinase signaling modules by scaffold proteins in yeast and mammals. Trends Biochem Sci 23:481-485. 
Wichterle H, Garcia-Verdugo JM, Herrera DG, Alvarez-Buylla A (1999) Young neurons from medial ganglionic eminence disperse in adult and embryonic brain. Nat Neurosci 2:461466.

Wichterle H, Turnbull DH, Nery S, Fishell G, Alvarez-Buylla A (2001) In utero fate mapping reveals distinct migratory pathways and fates of neurons born in the mammalian basal forebrain. Development 128:3759-3771.

Woodruff A, Xu Q, Anderson SA, Yuste R (2009) Depolarizing effect of neocortical chandelier neurons. Front Neural Circuits 3:1-15.

Woodruff AR, McGarry LM, Vogels TP, Inan M, Anderson SA, Yuste R (2011) State-dependent function of neocortical chandelier cells. J Neurosci 31:17872-17886.

Xu H, Jeong HY, Tremblay R, Rudy B (2013) Neocortical somatostatin-expressing GABAergic interneurons disinhibit the thalamorecipient layer 4. Neuron 77:155-167.

Xu Q, Cobos I, De La Cruz E, Rubenstein JL, Anderson SA (2004) Origins of cortical interneuron subtypes. J Neurosci 24:2612-2622.

Xu Q, Tam M, Anderson S (2008) Fate mapping Nkx2.1-Lineage cells in the mouse telencephalon. J Comp Neurol 506:16-29.

Yamasaki T, Kawasaki H, Arakawa S, Shimizu K, Shimizu S, Reiner O, Okano H, Nishina S, Azuma N, Penninger JM, Katada T, Nishina H (2011) Stress-Activated Protein Kinase MKK7 Regulates Axon Elongation in the Developing Cerebral Cortex. J Neurosci 31:16872-16883.

Yamasaki T, Kawasaki H, Nishina H (2012) Diverse Roles of JNK and MKK Pathways in the Brain. J Signal Transduct 2012:1-9.

Yang DD, Conze D, Whitmarsh AJ, Barrett T, Davis RJ, Rincon M, Flavell RA (1998) Differentiation of CD4+ T cells to Th1 cells requires MAP kinase JNK2. Immunity 9:575585.

Yau H, Wang H, Lai C, Liu F (2003) Neural development of the neuregulin receptor ErbB4 in the cerebral cortex and the hippocampus : preferential expression by interneurons tangentially migrating from the ganglionic eminences. Cereb Cortex 13:252-264.

Yokota Y, Ghashghaei HT, Han C, Watson H, Campbell KJ, Anton ES (2007) Radial glial dependent and independent dynamics of interneuronal migration in the developing cerebral cortex. PLoS One 2:e794.

Zecevic N, Hu F, Jakovcevski I (2011) Cortical interneurons in the developing human neocortex. Dev Neurobiol 71:18-33.

Zhang F, Yu J, Yang T, Xu D, Chi Z, Xia Y, Xu Z (2016) A novel c-Jun N-terminal kinase (JNK) signaling complex involved in neuronal migration during brain development. J Biol Chem 291:11466-11475.

Zhe J, Chenghai Z, Xiaorui H, Yaxin H (2012) Wnt5a promotes ewing sarcoma cell migration through upregulating CXCR4 expression. BMC Cancer 12:1-10.

Zimmer G, Garcez P, Rudolph J, Niehage R, Weth F, Lent R, Bolz J (2008) Ephrin-A5 acts as a repulsive cue for migrating cortical interneurons. Eur J Neurosci 28:62-73. 


\title{
Curriculum Vitae
}

\section{Abigail K. Myers}

567 Kristina Circle

State College, PA 16803

(814) 571-4136

Myersak2@gmail.com

\section{Education}

\author{
Doctor of Philosophy in Neuroscience, May 2017 \\ West Virginia University, Morgantown, WV \\ Mentor: Eric S. Tucker, PhD \\ Certificate in University Teaching, May 2014 \\ West Virginia University, Morgantown, WV \\ Masters of Science in Biomedical Sciences, May 2010 \\ Lake Erie College of Osteopathic Medicine, Erie, PA \\ Mentor: Randy J. Kulesza, PhD \\ Post Baccalaureate Program, April 2009 \\ Lake Erie College of Osteopathic Medicine, Erie, PA \\ Bachelor of Science in Kinesiology, August 2006 \\ Pennsylvania State University, University Park, PA
}

\section{Published Papers}

Cortical interneurons require Jnk1 to enter and navigate the developing cerebral cortex Myers, A.K., Meechan, D.W., Adney, D.R., Tucker, E.S.

J. Neurosci. 2014 Jun 4;34(23):7787-801.

- $\quad$ Selected for cover art

Prenatal diagnosis of congenital femoral deficiency and fibular hemimelia Radler C., Myers A.K., Hunter, R., Arrabal P.P., Herzenberg J.E., Grill F.

Prenat. Diagn. 2014 Oct; 34(10): 940-945.

Neonatal conductive hearing loss disrupts the development of the Cat-315 epitope on perineuronal nets in the rat superior olivary complex.

Myers A.K., Ray J., Kulesza R.J. Jr.

Brain Res. 2012 Jul 17;1465:34-47.

Maternal attitudes towards prenatal diagnosis of idiopathic clubfoot.

Radler C., Myers A.K., Burghardt R.D., Arrabal P.P., Herzenberg J.E., Grill F. Ultrasound Obstet Gynecol. 2011 Jun;37(6):658-62.

\section{Accepted Abstracts}

JNK Signaling Maintains the Integrity of Cortical Interneuron Migratory Streams During Corticogenesis

Myers, A.K., Baker, K., Snow, J.P, Hickling, S.E., Smith, C.A., Tucker, E.S

- Society for Neuroscience, Chicago, II

- 2015 Poster Presentation 
Cortical Interneurons Require JNK Signaling to Preserve Migratory Streams During Corticogenesis

Myers, A.K., Baker, K., Snow, J.P., Smith, C.A., Tucker, E.S.

- Van Liere Research Day, West Virginia University, Morgantown, WV

- 2015 Oral Presentation, awarded first place

Cortical Interneurons Require Jnk1 to Enter and Navigate the Developing Cerebral Cortex Myers, A.K., Meechan, D.W., Adney, D.R., Tucker, E.S.*

- Gordon Research Conference, Neural Development, Salve Regina University, Newport, RI

○ 2014 Poster Presentation

Cortical Interneurons Require JNK Signaling to Preserve Migratory Streams During Corticogenesis

Myers, A.K., Snow, J.P., Tucker, E.S.

- Van Liere Research Day, West Virginia University, Morgantown, WV

- 2014 Poster Presentation, awarded second place

Cortical Interneurons Require C-Jun N-Terminal Kinase Signaling to Enter and Navigate the Developing Cerebral Cortex

Myers, A.K., Reilly, E.C., Meechan, D.W., PhD, Adney, D.R., LaMantia, A-S., Tucker, E.S.*

- Gordon Research Conference, Neural Development, Salve Regina University, Newport, RI

- 2012 Poster Presentation

Cortical Interneurons Require C-Jun N-Terminal Kinase Signaling to Enter and Navigate the Developing Cerebral Cortex

Myers, A.K., Reilly, E.C., Meechan, D.W., Adney, D.R., LaMantia, A-S., Tucker, E.S.

- Appalachian Regional Cell Conference, Charleston, WV

- 2012 Poster Presentation, awarded Best in Group Poster Presentation

Cortical Interneurons Require Jnk1 and Jnk2 to Enter and Navigate the Cerebral Cortex Myers, A.K. ${ }^{\dagger}$, Tucker, E.S.*, Meechan, D.W., Adney, DR, LaMantia, A-S

- Society for Neuroscience, Washington, DC and San Diego, CA

- $2011^{*}, 2013^{\dagger} .2014^{\dagger}$ Poster Presentation

Jun Kinase Signaling is Required for Interneuron Migration into the Cerebral Cortex Myers, A.K., Tucker, E.S., Meechan, D.W., Adney, D.R., LaMantia, A-S.

- Van Liere Research Day, West Virginia University, Morgantown, WV

- 2012 Poster Presentation

Development of Perineuronal Nets in the Rat Auditory Brainstem

Myers, A.K., Kulesza, R.J. Jr.

- Lake Erie College of Osteopathic Medicine (LECOM) Research Day, Erie, PA

- 2009 Poster Presentation

Relapse of Idiopathic Clubfoot After the Ponseti Method

Myers, A.K., Burghardt, R., Sivaramalingam, S., Lamm, B.M.*, Herzenberg, J.E.

- American College of Foot and Ankle Surgeons Annual Meeting

- 2009 Oral Presentation, ${ }^{*}$ Honorable Mention Award

Maternal Experience with the Prenatal Diagnosis of Idiopathic Clubfoot

Radler, C., Myers, A.K., Burghardt, R., Arrabal, P.P., Herzenberg, J.E. ", Grill, F. 
- Pediatric Orthopedic Society of North America (POSNA) Annual Meeting

- 2009 Oral Presentation*

- American Orthopedic Association (AOA) Annual Meeting

- 2009 Poster Presentation*

\section{Invited Lectures}

Cortical Interneurons Require c-Jun N-Terminal Kinase (JNK) Signaling to Preserve Migratory Stream Integrity during Corticogenesis

November 21, 2014

Lake Erie College of Osteopathic Medicine, Erie, PA

The Role of JNK Signaling in the Developing Cortex

March 10, 2014

Lake Erie College of Osteopathic Medicine, Erie, PA

\section{Teaching Experience}

Graduate Medical Neuroanatomy, NBAN 793B

Spring 2015

Neurobiology and Anatomy, West Virginia University, Morgantown, WV

- Instructor

- Assisted with gross brain labs

- Lectures: Autonomic Nervous System, Connectopathy, Emotion

Cellular Methods, BMS 706

Fall 2012-Fall 2015

Biomedical Sciences. West Virginia University, Morgantown, WV

- Instructor, Model Systems (2012-2014)

- Created a lesson, homework, and review clicker questions

- Instructor, Immunohistochemistry and Microscopy (2014)

- Journal club facilitator (2015)

\section{Anatomy and Physiology 2, NBAN 207}

Fall 2012

Neurobiology and Anatomy, West Virginia University, Morgantown, WV

- Laboratory Teaching Assistant

- Assisted students at the cadavers by helping them locate structures and understand functions of identified organs and tissues

\section{Neuroanatomy}

Spring 2014, 2015

Anatomy, Lake Erie College of Osteopathic Medicine, Erie, PA

- Instructor, Basic Neurohistology

Honors \& Awards

- Created a lesson and exam questions

WVU Foundation Distinguished Doctoral Scholarship

2016 West Virginia University, Morgantown, WV

First Place in Oral Presentation

2015 Van Liere Research Day, West Virginia University, Morgantown, WV 
Second Place in Poster Presentation

2014 Neuroscience Retreat, Oglebay Resort, Wheeling, WV

Second Place in Poster Presentation

2014 Van Liere Research Day, West Virginia University, Morgantown, WV

Best in Group Poster Presentation

2012 Appalachian Regional Cell Conference, Charleston, WV 